University of Louisville

ThinkIR: The University of Louisville's Institutional Repository

$12-2018$

\title{
Understanding the relationship between anti-transgender bias, non-affirmation, and post-traumatic stress: a model of internalized transphobia-mediated post-traumatic stress.
}

Sebastian Mitchell Barr

University of Louisville

Follow this and additional works at: https://ir.library.louisville.edu/etd

Part of the Counseling Psychology Commons

\section{Recommended Citation}

Barr, Sebastian Mitchell, "Understanding the relationship between anti-transgender bias, non-affirmation, and post-traumatic stress: a model of internalized transphobia-mediated post-traumatic stress." (2018). Electronic Theses and Dissertations. Paper 3106.

https://doi.org/10.18297/etd/3106

This Doctoral Dissertation is brought to you for free and open access by ThinkIR: The University of Louisville's Institutional Repository. It has been accepted for inclusion in Electronic Theses and Dissertations by an authorized administrator of ThinkIR: The University of Louisville's Institutional Repository. This title appears here courtesy of the author, who has retained all other copyrights. For more information, please contact thinkir@louisville.edu. 
UNDERSTANDING THE RELATIONSHIP BETWEEN ANTI-TRANSGENDER

BIAS, NON-AFFIRMATION, AND POST-TRAUMATIC STRESS: A MODEL OF INTERNALIZED TRANSPHOBIA-MEDIATED POST-TRAUMATIC STRESS

\author{
By \\ Sebastian Mitchell Barr \\ B.A., Smith College, 2010 \\ Dissertation Submitted to the Faculty of the \\ College of Education and Human Development of the University of Louisville \\ in Partial Fulfillment of the Requirements \\ for the Degree of \\ Doctor of Philosophy

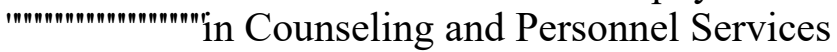

Department of Counseling and Human Development

University of Louisville

Louisville, Kentucky

December 2018 

UNDERSTANDING THE RELATIONSHIP BETWEEN ANTI-TRANSGENDER BIAS, NON-AFFIRMATION AND POST-TRAUMATIC STRESS: A MODEL OF INTERNALIZED TRANSPHOBIA-MEDIATED POST-TRAUMATIC STRESS

\author{
By \\ Sebastian Mitchell Barr \\ B.A., Smith College, 2010 \\ A Dissertation Approved on
}

October 5, 2018

by the following Dissertation Committee:

Kate Snyder, Ph.D.

Mark Leach, Ph.D.

Jill Adelson, Ph.D.

Stephanie Budge, Ph.D.

Katy Hopkins, Ph.D. 


\section{DEDICATION}

This dissertation is dedicated to

\section{Eric Christopher Collins}

$$
(1998-2013)
$$

and to all whose lives have been unjustly marked by bias and non-affirmation. 


\section{ACKNOWLEDGMENTS}

The achievement of the completion of this dissertation is not mine alone. The research is primarily built upon and inspired by the work of female and Black clinicians and researchers who have documented and attempted to understand the experiences of marginalized and oppressed peoples, even when mainstream psychology rejected their efforts. The final document was directly shaped by the feedback of my dissertation chairs and committee - their commitment to this project is something for which I am exceptionally grateful. My own ability to conceptualize and engage critically with this material, to design and run the study, to understand its results, and to consider the clinical implications represents the culmination of years of learning. I owe much to a collection of mentors, both official and unofficial, that is too great in number to list here. Similarly, the number of people who supported me emotionally and spiritually through this multiyear effort is too great to include in full. That said, a handful deserve special recognition. It is hard to imagine that I would have completed this without Dr. Kate Snyder and her support, understanding, wisdom, patience, flexibility, and motivation. I hold gratitude to Dr. Stephanie Budge for creating a space for me and my peers to do trans-affirming psychological research at a time when the field had yet to fully acknowledge a need for such work, and for her mentorship, modeling, and advocacy in this realm. Dr. Eric Russ deserves credit for introducing me to the field of trauma psychology and psychotherapy, and for encouraging me to expand my understanding of the multitude of ways in which trauma impacts a survivor. I also want to thank my mentors at Cambridge Hospital / Cambridge Health Alliance, particularly the Victims of Violence team, for their 
significant roles in my development as a trauma-aware clinician and world citizen. My family and partner have been my cheerleaders and bedrocks in this process, joining me in enthusiasm during exciting times and lifting me up and feeding me (literally and figuratively) in more draining periods - thank you. My friends have shown up for me in countless ways and I am grateful to have never felt alone in this process.

Finally, I want to acknowledge the large and invaluable role the trans and gender diverse community played in this dissertation. In addition to all that the community has done for me personally, the power and beauty of trans and gender diverse people has been a source of daily inspiration in my research. Over the past few years, when I have wanted to give up on this project, trans and gender diverse folks' stories and spirit reminded me that more work needs to be done to understand and reduce my community's distress and to understand and harness all of its strength. The "data" that I collected and analyzed for this dissertation are the lived experiences of trans people, the majority of whom are strangers to me, who volunteered their time and emotional labor to contribute to the literature. Reflecting on and reporting one's psychological suffering is an incredibly difficult task that should not be treated casually, especially given the field of psychology's history of pathologizing gender diversity and the possible negative associations and memories participants may have with the tasks I asked of them. I want to particularly recognize the efforts of participants who sit at the intersections of oppression - trans people of color, trans immigrants, trans people with severe mental illness and chronic illness or disability. Additionally, it must be said that most of what I have "found" and discussed in academic and clinical language in the next hundred or so pages are truths that have long been spoken by trans people but fallen on transphobic and cissexist ears. I am proud of the work I have done, but I also wish it were not as 
necessary. I want to state clearly that the research findings in this dissertation are less important than the voices of trans and gender diverse people themselves. 


\begin{abstract}
UNDERSTANDING THE RELATIONSHIP BETWEEN ANTI-TRANSGENDER BIAS, NON-AFFIRMATION AND POST-TRAUMATIC STRESS: A MODEL OF INTERNALIZED TRANSPHOBIA-MEDIATED POST-TRAUMATIC STRESS
\end{abstract}

\author{
Sebastian Mitchell Barr
}

October 5, 2018

Transgender people's increased risk for negative mental health outcomes, when compared to cisgender peers or the general public, has been well documented in the psychological literature. Researchers have begun to establish empirical support for a relationship between anti-transgender bias, non-affirmation of gender identity, internalized transphobia, and other transgender-specific minority stressors. Although little work has explored the mechanisms of this relationship, some psychologists have proposed conceptualizing these factors as potentially traumatic experiences and understanding the poor mental health outcomes as manifestations of complex posttraumatic stress. In this dissertation, I examine whether there is empirical support for this framework by evaluating the relationships between anti-transgender bias experiences, non-affirmation, internalized transphobia, and severity of symptoms of post-traumatic disorder. In Chapter 1, I review the literature on transgender mental health and conduct an in-depth exploration of the field of trauma psychology, offering critical reflections on the history of the field as well as the current etiological models of post-traumatic stress, while considering how these might apply to transgender mental health. Drawing primarily from feminist and multicultural theories of trauma, as well as theories of 
shame-based post-traumatic stress, I develop a theoretical framework supporting my model in which: a) non-affirmation and anti-transgender bias experience are related to the severity of PTSD symptoms, and b) this relationship is mediated by experiences of internalized transphobia. In Chapter 2, I discuss the methodology I utilized to assess my research questions, noting recruitment strategies, psychometric properties of measures I selected, and appropriateness of the analytic method of structural equation modeling. In Chapter 3, I present the results, which include the main findings that all hypothesized relationships were found to be significant: greater levels of non-affirmation and exposure to anti-transgender bias were related to greater levels of PTSD symptom severity, both indirectly through internalized transphobia and directly. In Chapter 4, I discuss my findings in the context of my theoretical framework and literature review, offer clinical and research implications, and caution readers about the limitations of this study. 


\section{TABLE OF CONTENTS}

ACNOWLEDGMENTS

ABSTRACT

LIST OF TABLES

LIST OF FIGURES

PAGE

iv

vii

$\mathrm{xi}$

xii

STATEMENT OF PROBLEM \& REVIEW OF THE LITERATURE

The Experiences and Mental Health of Transgender People 2

Defining Trauma and Conceptualizing Post-Traumatic Stress (PTS) 9

The Role of Shame and Internalized Stigma in Trauma 47

Shame Experienced by Transgender People and Its Role in PTS 54

$\begin{array}{ll}\text { Summary } & 58\end{array}$

$\begin{array}{ll}\text { Current Study } & 61\end{array}$

$\begin{array}{ll}\text { METHODOLOGY } & 63\end{array}$

Data Collection and Sample Demographics 63

$\begin{array}{ll}\text { Measures } & 67\end{array}$

$\begin{array}{ll}\text { Analytic Strategy } & 73\end{array}$

$\begin{array}{ll}\text { RESULTS } & 82\end{array}$

Descriptives and Frequencies $\quad 82$

Building the Measurement Model $\quad 84$

Building the Hybrid Model 86

$\begin{array}{ll}\text { Model Estimation } & 87\end{array}$

$\begin{array}{ll}\text { DISCUSSION } & 91\end{array}$ 
Difference Between Anti-Transgender Bias and Non-Affirmation

High Rates of PTSD Symptoms and Trauma Exposure

The Roles of Anti-Transgender Bias Experiences and Non-Affirmation

in PTS

The Mediating Role of Internalized Transphobia

Comparison of Non-Affirmation and Anti-Transgender Bias Experiences

100

Study Limitations and Future Research Directions

101

Clinical Implications

102

Conclusion

108

REFERENCES

110

APPENDIX A

CURRICULUM VITAE

141 


\section{LIST OF TABLES}

\section{TABLE}

PAGE

1. DSM-5 Diagnostic Criteria for PTSD 17

2. Root's (1992) Dimensions of Security 31

3. Sample Demographics 66

4. Trauma Exposure $\quad 83$

5. Model Fit Indices for Competing Factor Structures of the PCL-5 84

6. Model Fit Indices for Measurement Models 86

7. Model Results $\quad 89$

$\begin{array}{ll}\text { 8. Variable Means } & 140\end{array}$

9. Estimated Correlation Matrix for Latent Variables 140 


\section{LIST OF FIGURES}

FIGURE

1. 4-Factor PCL-5 Model

2. 6-Factor PCL-5 Model

3. 7-Factor PCL-5 Model

4. A Priori Measurement Model

5. Final Hybrid Model with Standardized Path Estimates
PAGE 


\section{CHAPTER I}

\section{STATEMENT OF THE PROBLEM \& REVIEW OF THE LITERATURE}

Transgender people ${ }^{1}$ are individuals whose gender identity does not fully align with the sex they were assigned at birth (APA, 2015). Transgender people are frequently victims of anti-transgender bias (Mizock \& Lewis, 2008), and these experiences are associated with a host of negative mental health outcomes, including depressive symptoms, anxiety, suicidality, and post-traumatic stress (e.g., Bockting, Miner, Swineburne Romine, Hamilton, \& Coleman, 2013; Clements-Nolle, Marx, \& Katz, 2006; Friedriksen-Goldsen et al., 2014; Shipherd et al., 2014). Conceptualizing anti-transgender bias experiences as trauma is helpful in understanding the relationships between such experiences and transgender people's mental health (Richmond, Burnes, \& Caroll, 2012), and research has documented that transgender people who have experienced more perceived discrimination report greater PTSD symptom severity than those who experienced less perceived discrimination (Reisner et al., 2016). No research, however, has explored the possible mechanisms between these bias events and PTSD symptoms. A clearer understanding of how anti-transgender bias events operate as potentially traumatic events and lead to post-traumatic stress is needed to help clinicians better promote psychological health and wellbeing in transgender clients (Richmond et al., 2012).

\footnotetext{
${ }^{1}$ Although I will be discussing transgender people using third person, I want to acknowledge that I do in fact identify as transgender and belong to this community.
} 
Building upon the Minority Stress Model (Hendricks \& Testa, 2012; Meyer, 2003), multiple theories of trauma (e.g., Bryant-Davis \& Ocampo, 2005; Foa \& Cahill, 2001; Root, 1992), and work on shame and internalized stigma (e.g., Tangney \& Dearing, 2002), I will examine the relationships between anti-transgender bias events, internalized transphobia, and PTSD symptoms.

In this chapter, I provide the foundation for my proposed model of antitransgender bias-based PTSD, drawing from psychological theory, empirical evidence, the voices of transgender people, and history. First, I provide an introduction to transgender people by briefly defining transgender identity, then exploring the literature on transgender mental health and experiences. Second, I introduce a broad conceptual framework through which we can understand transgender people's trauma. I do this by reviewing the history of the psychological study of trauma and exploring multiple modern conceptualizations of trauma, while identifying how transgender people's trauma experiences fit within current models. Third, I examine the role of shame and internalized stigma in trauma. Finally, I review literature on transgender people's shame experiences (specifically, internalized transphobia), and propose the previously unstudied role of these shame experiences in transgender people's development of post-traumatic distress.

\section{The Experiences and Mental Health of Transgender People}

A transgender person is an individual whose gender identity is different from the sex they were assigned at birth. Gender identity is a deeply held, internal sense of one's gender, while sex assignment that occurs at birth is typically based on genitalia (APA, 2015). Having a gender identity that differs from one's sex assignment is not rare, though it is uncommon. Most recent estimates of the proportion of United States residents who identify as transgender place the number at .6\% (Flores, Herman, Gates, \& Brown, 2016), 
meaning 1.4 million Americans identify as transgender. The actual figure is likely to be larger (Meier \& Labuski, 2013). The term transgender is typically considered an umbrella term that includes a range of gender identities and expressions. Importantly, gender identity is increasingly understood as a nonbinary construct, meaning that someone may identify their gender as falling along a continuum between man or woman, or as an alternative gender unrelated to the categories of man and woman (APA, 2015).

Transgender people may be more likely to seek psychotherapy than nontransgender (also referred to as cisgender) people (Carmel, Hopwood, \& dickey, 2014). This increased need for services is likely due to multiple factors: First, historically, transgender people were required to seek mental health evaluation and treatment to be approved for transition health care (Hopwood \& dickey, 2014); second, transgender people may utilize psychotherapy to aid in their identity development/acceptance work (Bockting \& Coleman, 2008); and third, transgender people are more likely than the general population to struggle with their mental health (Carmel, Hopwood, \& dickey, 2014).

Research on transgender people's mental health illustrates that transgender people face great risk for mental health and psychological difficulties. Studies have found rates of psychiatric diagnoses in transgender samples that are significantly higher than those found in epidemiological studies of the general population (e.g., Hepp, Kraemer, Schnyder, Miller, \& Delsignore, 2005; Terada et al., 2012). The largest known study of transgender people's mental health, using online data collection, found that in a sample of over one thousand transgender women and men, $44 \%$ met criteria for clinically significant depression, $33 \%$ for clinically significant anxiety, and $38 \%$ for somatization (Bockting, Miner, Romine, Hamilton, \& Coleman, 2013). In a separate study, when a 
sample of transgender ${ }^{2}$ women and men were compared with matched non-transgender controls, transgender people showed increased psychopathology (measured by the Symptom Check List-90-Revisited Instrument; SCL-90-R) both when compared to nontransgender controls of the same gender and when compared to non-transgender controls of the same sex assigned at birth (Auer et al., 2013). Transgender people consistently demonstrate levels of anxiety and depression symptoms that are greater than those found in the general population (e.g., Budge, Adelson, \& Howard, 2013), and are more likely than the general population to meet the criteria for post-traumatic stress disorder (PSTD; Shipherd et al., 2014). In comparisons to the general population, studies have also demonstrated increased suicidality in transgender populations (e.g., Blosnich et al., 2013; Haas, 2014), increased self-harm behavior (dickey, Reisner, \& Juntunen, 2015), and increased interpersonal difficulties (Davey, 2015). One study has contradicted these findings and reported that the differences in current symptomology and diagnoses between transition-seeking transgender people and non-transgender people were only small, though statistically significant, and may not represent meaningful difference (Fisher et al., 2012). The study was conducted at a gender transition clinic, and the authors explained their unexpected findings as a consequence of the defensiveness that transgender people present with when seeking transition-related healthcare. Indeed, transgender people wary of losing access to a gender transition often downplay psychiatric symptoms and psychological distress (Hopwood \& dickey, 2014), which suggests that data collected in this manner are less likely to yield reliable results and lead

${ }^{2}$ Auer et al. (2013) referred to their participants as transsexuals; their use of that label was motivated by the fact that these individuals had a diagnosis of Gender Identity Disorder (which has been updated and replaced by Gender Dysphoria), not participants' self-identification with the "transsexual" label. Transgender is the more appropriate terminology for such individuals and the use of this term does not alter their conclusions. 
to valid conclusions. There are two main sources of the documented mental health disparities transgender people face: gender dysphoria and anti-transgender bias (Carmel, Hopwood, \& dickey, 2014).

\section{Gender Dysphoria}

Gender dysphoria is the distress that some transgender people experience due to the incongruences between their body and their gender identity and between others' perceptions of their gender and their gender identity (APA, 2015). Treatment for gender dysphoria involves social and/or medical gender transitions to reduce the felt incongruence. Transgender people consistently report that transition reduces their gender dysphoria and related psychological distress (Erickson-Shock, 2015). Empirical evidence supports this: in the only longitudinal study currently published, a sample of transgender men showed significant declines in MMPI-2 psychopathology within the first year of testosterone treatment (Keo-Meier et al., 2015). Cross-sectional studies have also found that individuals who are further progressed in their transition have better mental health and psychological functioning (e.g., Barr, Budge, \& Adelson, 2016; Budge et al., 2013).

\section{Anti-transgender Bias}

Anti-transgender bias is a common, but harmful experience for transgender people (Bockting et al., 2013). Transgender people are likely to have experienced discrimination or rejection due to being transgender or gender nonconforming, and a large proportion of transgender people experience harassment and violence that stem from their gender identity or presentation (Bazargan \& Galvan, 2012; Bradford, Reisner, Honnold, \& Xavier, 2013; Dispenza, Watson, Chung, \& Brack, 2012; Grant et al., 2011;

Mizock \& Lewis, 2008). Anti-transgender bias is also experienced as non-affirmation of a transgender person's gender identity, which could include “assuming a person's 
assigned sex at birth is fully aligned with that person's gender identity, not using a person's preferred name or pronouns, asking [transgender or gender nonconforming people] inappropriate questions about their bodies, or making the assumption that psychopathology exists given a specific gender identity or expression" (APA, 2015, p. 10; Nadal, Rivera, \& Corpus, 2010; Nadal, Skolnik, \& Wong, 2012). Experiences of bias and stigmatization are associated with a range of negative mental health outcomes, including general psychological distress, suicidality, and self-injury (Bockting et al., 2013; dickey, Reisner, \& Juntunen, 2015; Goldblum et al., 2012; Nuttbrock et al., 2010; Testa et al., 2012; Testa et al., 2015). Transgender individuals who face greater discrimination also report greater levels of PTSD than transgender people who face less discrimination, even after controlling for exposure to traditionally defined traumas (Reisner et al., 2016).

The negative impact of anti-transgender bias on transgender people's mental health is currently understood through Hendricks and Testa's (2012) adaptation of the Minority Stress Model (Meyer, 2003). Anti-transgender bias that is experienced as an external event is conceptualized as distal minority stress. Hendricks and Testa (2012) defined four types of distal minority stressors for transgender people: gender-related discrimination, gender-related rejection, gender-related victimization, and gender-related non-affirmation. These stressors lead to increased psychological distress. Distal minority stressors also increase a person's experience of internal or more proximal minority stressors (Meyer, 2003), which Hendricks and Testa (2012) defined for transgender people as internalized transphobia, expectation of discrimination and rejection in future events, and nondisclosure (or concealment of transgender identity or history). The Minority Stress Model theorizes that proximal minority stressors partially mediate the 
relationship between distal minority stressors and mental health outcomes (Hendricks \& Testa, 2012; Meyer, 2003). Research documenting a relationship between trans-specific proximal stressors and psychological distress has provided support for this theory (Testa et al., 2015). Of all the proximal stressors, internalized transphobia is the best understood, and is reviewed later.

\section{Trauma and PTSD}

A disparity also has been documented with regard to prevalence of post-traumatic stress disorder (PTSD). In a study of transgender adults who were male assigned at birth, $17.5 \%$ of the full sample endorsed DSM-IV PTSD symptoms at a clinically significant level (Shipherd, Maguen, Skidmore, \& Abramovitz, 2011). This proportion is markedly larger than the DSM-IV PTSD prevalence rates of the general population (5\% to $10 \%$; Kessler et al., 1995). The same study found a high rate of exposure to traditionally defined trauma: $98 \%$ of participants had experienced at least one potentially traumatic Criterion A event (PTE), as measured by the commonly-used Traumatic Life Events Questionnaire (TLEQ; Kubany et al., 2000), and 91\% reported having experienced multiple PTEs. The overall trauma exposure rate in this sample is higher than that of the general population (e.g., Breslau, 1998), but transgender people's exposure to interpersonal violence is particularly high. Estimates of the proportion of transgender people who have experienced physical assault range from $25 \%$ to $51 \%$ (Nuttbrock et al., 2010; Shipherd et al., 2014; Testa et al., 2012). Estimates of the proportion of transgender people who have been the victims of sexual assault range from $6 \%$ to $27 \%$ (Shipherd et al., 2014; Testa et al., 2012). ${ }^{3}$ Transgender people's increased exposure to violence is

\footnotetext{
${ }^{3}$ Note that the research on trauma exposure and PTSD prevalence in transgender populations has utilized disproportionately White samples; transgender people of color experience more bias and may have even higher rates of exposure to PTEs and bias-
} 
often due to anti-transgender bias. Forty-two percent of Shipherd and colleague's (2014) transgender sample reported that at least one of their traumatic experiences was due to anti-transgender bias, and male-assigned-at-birth participants who more often presented as women were more likely to be victimized compared to those who presented as women less often. More than 50\% of Nuttbrock and colleagues' (2010) sample reported a history physical abuse that was directly related to the participant's gender identity or presentation. In addition to being associated with PTSD symptoms in transgender populations (Reisner et al., 2016; Shipherd et al., 2014), experiences of bias-related interpersonal trauma are also related transgender people's depression and suicidality (Clements-Nolle, Marx, \& Katz, 2006; Nuttbrock et al., 2010).

Recall that the aforementioned study by Reisner and colleagues (2016) found that after controlling for experiences of traditionally-defined traumas, such as interpersonal violence, greater levels of discrimination were associated with increased severity of PTSD symptoms in a transgender sample. Recently, psychologists have proposed understanding transgender people's exposure to anti-transgender bias through a conceptual framework rooted in trauma theory (Richmond, Burnes, \& Carroll, 2011). Richmond and colleagues argued that anti-transgender discrimination and other biasrelated experiences are forms of insidious trauma (Root, 1992) that can be considered experiences of violence; such experiences should thus be considered within a context of all the violence by which transgender people are victimized. Under this framework, transgender people's increased rates of psychopathology are the result of symptoms of traumatization. Beyond Reisner and colleagues' (2016) study linking discrimination

related PTEs, as well as higher rates of severe PTSD symptomology than their White peers (Mizock \& Lewis, 2008). 
experiences with transgender people's PTSD symptom severity, no known research has tested this trauma-based framework.

\section{Defining Trauma and Conceptualizing Post-Traumatic Stress}

Understanding transgender people's experiences of anti-transgender bias through a trauma framework requires a thorough understanding of trauma theory, the historical origins of such theory, and current debate over the definition of trauma and etiology of post-traumatic stress.

\section{Historical Context (Prior to DSM-III)}

Post-traumatic stress disorder (PTSD) is a modern construct and diagnosis, having first appeared in the American Psychiatric Association's (American Psychiatric Association, 1980) third edition of its Diagnostic and Statistical Manual of Mental Disorders (DSM-III). Trauma's impact on human behavior and psychological functioning, however, is documented well before the DSM-III. To understand the current challenges in defining PTSD and traumatic events, one is aided by understanding the history of trauma psychology; this history highlights the instability of the construct, as well a pattern of mistakes on the part of mainstream science and medicine, namely in attempts to define survivors' experiences for them (Herman, 1992). It will become particularly clear as the history is reviewed that it was not until the survivors of trauma had the power to speak out and be listened to that advancements in humane and effective research and treatment were actually made. As will be discussed later, in recent years, psychologists from marginalized communities (e.g., people of color, women, queer, transgender psychologists) have developed a substantial body of theory and empirical evidence supporting an expansion of trauma to include bias events (e.g., Bryant-Davis \& 
Ocampo, 2005). Little of this work has been incorporated into mainstream trauma teaching, research, or treatment.

Railway accidents. Trauma first entered the literature of Western medicine in the latter half of the $19^{\text {th }}$ century, amidst a decades-long wave of devastating railway accidents (Weisath, 2014). Surgeons who treated passengers injured in these crashes and derailments noted symptoms that matched what we today might expect from a trauma survivor. However, in attempts to distinguish male railway accident survivors from "weak" females suffering from hysteria, some surgeons proposed that accident victims" behavioral symptoms were the result of a physical "shock to the system," which resulted in a spinal concussion (Erichsen, 1866). Other surgeons challenged this position, attributing emotional disturbances to "mental shock" or "fright" (Page, 1883, p. 169). In her study of the history of trauma psychology, Herman (1992) noted that this question of whether the origin of trauma victims' symptoms was physical or psychological would reemerge in the field's subsequent efforts to treat hysteria and war neuroses.

Hysteria. Historians often attribute the "birth of trauma" and its study (Fassin \& Rechtman, p. 30) to the study of hysteria (e.g., Libbrecht \& Quackelbeen, 1995; Van Der Kolk, 2007; Weisaeth, 2002). By the late $19^{\text {th }}$ century, the commonly acknowledged forefathers of psychology were working in Europe to comprehend the broad category of emotional and behavioral disturbances labeled hysteria. Jean-Martin Charcot had made his career recording the symptoms and physical characteristics of female hysterics (as they were called at the time; Weisaeth, 2014). Toward the end of his career, Charcot, inspired by the work with male railway accident survivors, began studying so-called male hysterics and proposed an etiological link between trauma experiences and dissociation and other behavioral symptoms (Charcot, 1887, as cited in Van Der Kolk, 2007). 
Charcot's students expanded this understanding of trauma and applied it to females with hysteria symptoms. Herman (1997) asserted that famed psychologists Janet, Freud, and Breuer, made the first documented efforts to speak with women suffering from hysteria as a method of empirical research. In doing so, they all came to the conclusion that the origin of female hysterics' suffering was traumatic memories, referred to by Janet (1984, as cited in Van Der Kolk, 2007) as subconscious fixed ideas and by Freud and Breuer (1895) as reminiscences. Freud (1896) specifically attributed the development of hysteria to repressed memories of childhood sexual abuse. As these men provided some temporary validity to the suffering of hysterics, more women came forward with symptoms of hysteria. Letters from Freud suggest that he was overwhelmed by the number of women suffering and found it beyond belief that the violations he had believed to be the source of hysteria could be so commonplace (Herman, 1997). Van der Kolk (2007) also noted that new leaders in medicine at the turn of the century were dismissive of the theories positing psychical trauma as a possible origin of hysteria. Freud and his peers accordingly moved away from their initial understanding of hysteria, and the emerging field of psychology adopted a view of women's mental illness framed by assumptions of malingering, false memories, and subconscious desires to be victims of sexual trauma (Freud, 1925; Westerland, 1986). Scientists and physicians lost interest in hysteria, and it would be more than half a century before the field recognized the validity of these women's suffering and the sources of such suffering (Burgess, 1983; Herman, 1992; Westerland, 1986).

War neuroses, the antiwar movement, and combat trauma. World War I brought a renewed focus to trauma. During the war, medics in the military could not make sense of the overwhelmingly large number of soldiers experiencing combat-related 
syndromes. Initially, these syndromes were described as physical in nature. As the psychic nature of soldiers' conditions started to become unavoidable, the phrase "war neuroses" became commonplace (Weisaeth, 2002; 2014). However, because injuries of the psyche were considered within a person's control (at least to an extent far beyond bodily injuries), the adaptation of a psychological model of war neuroses meant that those suffering were viewed with contempt (Herman, 1997; Van der Kolk, 2007). They were considered moral invalids and leading psychiatrists employed and promoted 'treatment' grounded in shaming, threatening, and punishing (Leri, 1918). For example, in his thenconsidered-seminal text, Hysterical Disorders of Warfare, military psychiatrist Yealland (1918) documented the use for electric shock for treating what we would call psychosomatic symptoms, recommended that those whose 'nervousness' or 'depression of spirits' prevented them from returning to combat be court martialed, and described techniques that involved yelling at patients for being cowardly and lazy during hourslong sessions. W. H. R. Rivers is credited with shifting the approach to treating sufferers of war neuroses; he famously "cured" veteran Siegel Sassoon by kindly inviting him to talk about his experiences. However, the war soon ended and the veterans with psychological problems, considered a national embarrassment and still often thought of as weak-willed and unmasculine, spent the rest of their lives in mental institutions (Herman, 1997).

Little record of work on war neuroses in the literature exists between WWI and WWII (Weisaeth, 2014). The exception was psychiatrist Kardiner, who was troubled by his field's approach to combat veterans, particularly after the war. Dismayed, he left the field to study anthropology. With the return of war and with a heightened understanding of the impact of context on an organism, Kardiner returned to the study of war neuroses. 
He famously published a book in which he demonstrated that combat-induced mental illness was not due to weak will or poor character and could in fact happen to anyone (Kardiner, 1941; Van der Kolk, 2007). Military psychiatrists used Kardiner's work as a jump-off point and began investigating how to prevent men in combat from developing war neuroses. Research was also conducted on how to quickly treat these neuroses in a way that allowed for swift return to the front lines (Van der Kolk, 2007; Weisaeth, 2014). But again, the war ended and little attention was paid to the psychological impact of war for many years. There was some research in Europe on the psychological syndromes seen in Holocaust survivors (e.g., Krystal, 1968, 1978, 1988, as cited in Van der Kolk, 2007), but this too was short-lived and received almost no attention from American psychologists (Weisaeth, 2014). Even as psychological scientists and practitioners were beginning to establish the validity of psychological sources of suffering and the normalcy of stress responses to traumatic events, this work was done solely within the context of war traumas and for the purpose of finding and developing better fit soldiers.

An important shift in the way psychologists and American culture at large understood trauma occurred when veterans advocacy groups, not military physicians or great psychological thinkers, took the lead. As part of the anti-war protest efforts in the 1970s, Vietnam veterans began speaking publicly about the emotional and psychological impact the war had on them (Weisath, 2014). In addition to forcing public discourse on the far-reaching psychological effects of combat trauma, members of the group Vietnam Veterans against the War also organized grassroots treatment efforts. These veterans gathered in small rap groups, where they would process combat experiences and their difficulties returning to civilian life. They often invited progressive psychiatrists or psychologists to observe or facilitate the groups, and these clinicians noted the benefit of 
the groups and their apparent success in helping veterans cope with the trauma they had experienced (Herman, 1997). Growing public pressure, built and sustained by veterans' organizing efforts, their collaborations with psychologists and psychiatrists, and other antiwar movement work, to assist in veterans' recovery meant an increase in funding and research. This ultimately culminated in the proposal of a diagnosis for the DSM-III. The initial proposal, however, focused exclusively on war trauma (Herman, 1992).

Women's liberation and rape/abuse trauma. The fact that the field of psychology now understands PTSD as a somewhat broad diagnostic category inclusive of a variety of types of trauma, rather than a diagnosis specific to combat trauma, is largely due to the efforts of activists from the Women's Liberation Movement and female researchers and practitioners. While Vietnam veterans were using protest strategies to highlight the horrors of war abroad, women activists in the 1960s and 70s were organizing to raise awareness of the horrors of what Herman (1997) calls "the sex war" (p. 92): rape and other sexual victimization. Groups such as the National Organization for Women aimed to open the public's eyes to the commonality and devastating impact of rape by organizing speak-outs and rallies. This growing public conversation about rape and the increasing power of women in the social sciences coalesced, and for the first time, researchers began systematically studying the effects of rape on women. Ann Burgess and Lynda Holmstrom, a psychiatric nurse and a sociologist, respectively, published the first known results of such a study and determined that survivors of abuse often had similar symptoms and difficulties. They termed this "rape trauma syndrome" (Burgess \& Holmstrom, 1974). As researchers and practitioners began to legitimate the histories and suffering of women and children, more survivors came forward, and more research followed. The National Institutes of Mental Health opened a center for rape 
research, and lines of academic inquiry were opened into the effects of domestic violence and incest (Herman, 1997; Van der Kolk, 2007). In addition to identifying the commonalities between survivors of these types of violence, researchers noted the similarities in symptoms and behaviors between survivors of "civilian" violence and combat survivors. This work culminated in psychology's first general category of trauma and its psychological impact on human behavior and psychological functioning. Bringing the field of trauma psychology almost full circle, researchers at this time also noted the similarities between the historic cases of hysteria and the sexual trauma survivors diagnosed with PTSD (Herman, 1997). Nearly a century later, "hysterics" were recognized as traumatized individuals whose psychological distress and so-called abnormal behavior was retroactively acknowledged as valid human responses to their trauma (Burgess, 1983).

This history is critical to understanding current work in trauma psychology because it highlights the instability within the development of PTSD as a diagnostic label and psychological construct. Importantly, this history provides evidence that necessary growth toward more accurate and more helpful understandings of trauma and its impact have come from listening to survivors and outsiders.

In this paper, I will ultimately present a conceptualization of anti-transgender bias experiences as traumatic. Mainstream psychology currently lacks a frame for understanding how minority and stigmatized people are affected by oppression. Without proper attention to oppression-related factors, the psychological community misdiagnoses such individuals; this not only inappropriately pathologizes already stigmatized people, it also prevents appropriate intervention and allows mainstream society to ignore the impact of oppression (Brown, 2008; Herman, 1997). Much like the trauma theory advances in 
history, attending to the experiences of transgender people is likely to help move clinicians and researchers' understanding of trauma forward.

\section{Modern Understandings of Trauma and Post-Traumatic Distress}

Although the introduction of PTSD into the DSM-III established the validity and non-rarity of negatively impactful responses to trauma, the DSM-III did not solidify PTSD as a static construct. Over the past three decades, psychologists have proposed and explored multitudes of definitions and theories of trauma and post-traumatic stress. Each revision to the DSM has included partial revisions to PTSD's diagnostic criteria (Friedman, 2014). Today, the field of psychology remains far from consensus on both the diagnostic criteria of PTSD and a model of etiology (Friedman, Resick, Bryant, \& Brewin, 2011). In the following review of the field's current approaches to conceptualizing trauma and post-traumatic distress, I include discussion of the current diagnostic criteria in the DSM-5, as well as prominent theories of trauma from cognitivebehavioral, feminist, and multicultural psychologists. I also discuss the applicability of each approach to understanding transgender people's experiences of trauma and psychopathology.

Diagnostic criteria and associated debate. PTSD is listed in the current edition of the APA's (American Psychiatric Association, 2013) DSM-5 under Trauma- and Stressor-Related Disorders, a category that is new to this edition (Friedman, 2014). Full diagnostic criteria are presented in Table 1. The criteria require that a person, older than age 6 , have been exposed to actual or threatened death, serious injury, or sexual violence (Criterion A), and that following such exposure, they have experienced intrusion symptoms, persistent avoidance, negative alterations in cognitions and mood, and marked alterations in arousal and reactivity for at least one month; the disturbance must cause 
clinically significant distress or impairment and cannot be attributable to substance use or a medical condition. Though there remains debate about many components of the DSM-5 criteria (see Friedman et al., 2014a for review), the most prominent debates surround

Criterion A, or what is considered trauma exposure.

Table 1

DSM-5 Diagnostic Criteria for Posttraumatic Stress Disorder

Note: The following criteria apply to adults, adolescents, and children older than 6 years.

A. Exposure to actual or threatened death, serious injury, or sexual violence in one (or more) of the following ways:

1. Directly experiencing the traumatic event(s).

2. Witnessing, in person, the event(s) as it occurred to others.

3. Learning that the traumatic event(s) occurred to a close family member or close friend. In cases of actual or threatened death of a family member of friend, the event(s) must have been violent or accidental.

4. Experiencing repeated or extreme exposure to aversive details of the traumatic event(s) (e.g., first responders or collecting human remains; police officers repeatedly exposed to details of child abuse)

Note: Criterion A4 does not apply to exposure through electronic media, television, movies, or pictures, unless this exposure is work related.

B. Presence of one (or more) of the following intrusion symptoms associated with the traumatic event(s), beginning after the traumatic event(s) occurred:

1. Recurrent, involuntary, and intrusive distressing memories of the traumatic event(s).

Note: In children older than 6 years, repetitive play may occur in which themes or aspects of the traumatic event(s) are expressed.

2. Recurrent distressing dreams in which the content and/or affect of the dream are related to the traumatic event(s).

Note: In children, there may be frightening dreams without recognizable content.

3. Dissociative reactions (e.g., flashbacks) in which the individual feels or acts as if the traumatic event(s) were recurring. (Such reactions may occur on a continuum, with the most extreme expression being a complete loss of awareness of present surroundings.)

Note: In children, trauma-specific reenactment may occur in play.

4. Intense or prolonged psychological distress at exposure to internal or external cues that symbolize or resemble an aspect of the traumatic event(s).

5. Marked physiological reactions to internal or external cues that symbolize or resemble an aspect of the traumatic event(s).

C. Persistent avoidance of stimuli associated with the traumatic event(s), beginning after the traumatic event(s) occurred, as evidenced by one or both of the following: 
1. Avoidance of or efforts to avoid distressing memories, thoughts, or feelings about or closely associated with the traumatic event(s).

2. Avoidance of or efforts to avoid external reminders (people, places, conversations, activities, objects, situations) that arouse distressing memories, thoughts, or feelings about or closely associated with the traumatic event(s).

D. Negative alterations in cognitions and mood associated with the traumatic event(s), beginning or worsening after the traumatic event(s) occurred, as evidenced by two (or more) of the following:

1. Inability to remember an important aspect of the traumatic event(s) (typically due to dissociative amnesia and not to other factors such as head injury, alcohol, or drugs).

2. Persistent and exaggerated negative beliefs or expectations about oneself, others, or the world (e.g., "I am bad," "No one can be trusted," "the world is completely dangerous," "My whole nervous system is permanently ruined").

3. Persistent, distorted cognitions about the cause or consequences of the traumatic event(s) that lead the individual to blame himself/herself or others.

4. Persistent negative emotional state (e.g., fear, horror, anger, guilt, or shame).

5. Markedly diminished interest or participation in significant activities.

6. Feelings of detachment or estrangement from others.

7. Persistent inability to experiences positive emotions (e.g., inability to experiences happiness, satisfaction, or loving feelings).

E. Marked alterations in arousal and reactivity associated with the traumatic event(s), beginning or worsening after the traumatic event(s) occurred, as evidenced by two (or more) of the following:

1. Irritable behavior and angry outbursts (with little or no provocation) typically expressed as verbal or physical aggression toward people or objects.

2. Reckless or self-destructive behavior.

3. Hypervigilance.

4. Exaggerated startle response.

5. Problems with concentration.

6. Sleep disturbance (e.g., difficulty falling or staying asleep or restless sleep).

F. Duration of the disturbance (Criteria B, C, D, and E) is more than 1 month.

G. The disturbance causes clinically significant distress or impairment in social, occupational, or other important areas of functioning.

H. The disturbance is not attributable to the physiological effects of a substance (e.g., medication, alcohol) or another medical condition.

Specify whether:

With dissociative symptoms: The individual's symptoms meet the criteria for post-traumatic stress disorder, and in addition, in response to the stressor, the individual experiences persistent or recurrent symptoms of either of the following: 
1. Depersonalization: Persistent or recurrent experiences of feeling detached from, and as if one were an outside observer of, one's mental processes or body (e.g., feeling as those one were in a dream; feeling a sense of unreality of self or body or of time moving slowly).

2. Derealization: Persistent or recurrent experiences of unreality of surroundings (e.g., the world around the individual is experienced as unreal, dreamlike, distant, or distorted).

Note: To use this subtype, the dissociative symptoms must not be attributable to the physiological effects of substance (e.g., blackouts, behavior during alcohol intoxication) or another medical condition (e.g., Specify if: complex partial seizures).

With delayed expression: If the full diagnostic criteria are not met until at least 6 months after the event (although the onset and expression of some symptoms may be immediate).

It is made clear by both the name of the disorder itself (post-traumatic stress disorder) and its diagnostic criteria (Criterion A, specifically) that the symptoms described in Criterions B, C, and D only constitute PTSD if the affected individual experienced a traumatic event. Since the inception of the diagnosis (and arguably before, though with different goals), researchers and practitioners have been debating where to draw the lines around what constitutes a traumatic event vs. a non-traumatic event (Weisath, 2014). In the DSM-IV, Criterion A was divided into A1 (which described necessary characteristics of an event: involving "actual or threatened death or serious injury, or a threat to the physical integrity of self or others;" APA, 2000) and A2 (which described necessary emotional responses to the event: fear, helplessness, or horror). The task force charged with updating the diagnostic criteria for the DSM-5 removed the A2 criterion in the face of clear evidence that PTSD routinely develops in individuals who did not experience an A2-qualifying emotional response at the time of the traumatic event (Friedman \& Resick, 2014).

The task force also considered removing the A1 criterion in recognition of evidence that individual differences in psychological vulnerabilities leads to differences 
in perception of whether or not an event is life-threatening. A1 criterion was ultimately retained, with researchers citing McNally's (2009, as cited in Friedman \& Resick, 2014) conclusion that "the memory of the trauma is the 'heart of the diagnosis' and the organizing core around which the B-F symptoms [intrusion symptoms, avoidance symptoms/behaviors, negative alterations in mood, alterations in arousal, and a duration of at least one month] can be understood" (p. 23). The language in Criterion A was slightly expanded, but largely upheld Weathers and Keane's (2007) argument that a traumatic event entails "personal involvement with, if not direct exposure to catastrophic life events" (p. 115, as cited in Friedman \& Resik, 2014, p. 23). Much research, however contradicts the task force's assumption that a 'catastrophic life event' must be one that involves "exposure to actual or threatened death, serious injury, or sexual violence" in very specific ways (American Psychiatric Association, 2013). Many researchers have found associations between exposure to non-Criterion A events and increased PTSD diagnoses and/or symptomatology, even after controlling for the experience of Criterion A events (e.g., Carlson \& Dalenberg, 2000; Gold et al., 2005; Long et al., 2000; Mol et al.,2005; Van Hooff, 2009). As I will discuss later in my review of feminist and racismbased theories of trauma, an important area of research of the validity and appropriateness of Criterion A has examined the traumatic nature of incidents that are racist, misogynist, and otherwise oppressive. Many trauma psychologists have called on expansion of Criterion A to include prejudice events (e.g., Sanchez-Hucles, 1998). This is key for understanding many of transgender people's trauma experiences, as the insidious trauma of anti-transgender bias does not qualify as a Criterion A trauma but has been linked with PTSD symptoms and poor mental health (Bockting et al., 2013; Reisner et al., 2016). 
Additionally, feminist psychologists and supporters of other non-mainstream conceptualizations of trauma are critical of the entire notion that characteristics of an event should determine whether or not it qualifies as potentially traumatic. In summary of work by Allen (1996), Briere (2004), and Bryant-Davis and Ocampo (2005), Helms, Nicolas, and Green (2010) stated that for certain events, "it is the victimized person's subjective interpretation of the events that they experienced rather than the objective reality or physical properties of the event that determine whether it is traumatic" (p. 54). These theorists argue that the survivor's perception of an event and its impact on them are what is most important to the event's classification as traumatic or non-traumatic. The exact same event may be traumatic for one person and not for another. It should also be noted that some theorists whose work is discussed in this dissertation would object to the validity of even delineating specific diagnostic criteria. This objection is, in part, due to differences in fundamental approaches to making sense of human behavior and the impact of trauma, as well as the development of so-called psychopathology. These differences will be made clear in the following review of etiological theories.

Psychological theories of trauma. As stated earlier, the 1980 introduction of PTSD as a diagnosis represented a shift in the psychological community toward recognition of the symptoms of post-traumatic stress as valid and even normal human responses to trauma. However, while these PTSD responses are common, they are not universal. The etiological links between trauma exposure and PTSD and other traumarelated disorders are not direct; research even suggests that the majority of people exposed to trauma have never met the criteria for a PTSD diagnosis. Researchers attempting to estimate prevalence rates in the United States have found that between 37 and $92 \%$ of respondents had been exposed to trauma, with the wide range being largely 
due to differences in sample (Breslau, 1998). Recent epidemiological studies have estimated the lifetime prevalence of PTSD (utilizing DSM-5 criteria) as $6.1 \%$ (Goldstein, 2016). Even if that proportion is expanded to include subthreshold PTSD (with an average estimated prevalence rate of $12.6 \%$; Branco, 2016), these results indicate that natural recovery from trauma exposure without ever developing PTSD is possible. Clearly, factors beyond trauma exposure alone play contributing roles in the development of PTSD. Many etiological theories have been postulated to explain these mechanisms behind PTSD development following trauma exposure.

In this section, I review the two most prominent mainstream models of PTSD etiology: conditioning and emotion processing theories. I also discuss feminist theories of trauma and theories of racist-incident based trauma in depth; Richmond and colleagues' trauma framework for understanding transgender mental health and experiences of oppression is rooted in this work. This section also includes a brief review of schema theories of trauma, as these lay the foundation for the feminist trauma theories.

Conditioning models. Etiological theories of PTSD that involve conditioning focus on fear and anxiety and have their origin in Mowrer's (1960) two-factor theory. This theory proposed that anxiety develops through two factors: classical conditioning and operant conditioning; this idea was revolutionary during an era when psychologists were in the habit of pledging allegiance to either classical condition or operant conditioning as their school of psychology (Monson, Friedman, \& La Bash, 2014). Mowrer (1960) proposed that classical conditioning explained how individuals initially developed a fear response to seemingly non-threatening stimuli, but that operant conditioning explained how this was maintained, due to relief experienced by avoiding the conditioned stimuli. Kilpatrick, Veronen, and Resick (1979) were the first to apply 
the two-factor theory to the development of post-traumatic stress. They theorized that stimuli present during the traumatic event elicit fear-based cognitive, emotional, physiological, and behavioral responses when encountered in subsequent non-traumatic situations due to classical conditioning. These stimuli can be external (e.g., the sound of explosions at a fireworks show, sexual touch in consensual situations) or internal (e.g., cognitive representations of the traumatic event or conditioned external stimuli, emotions or physiological sensations that the survivor had also experienced during the trauma). Additionally, the fear response may be generalized and extend to stimuli that are only similar to those that were present during the traumatic event. Kilpatrick and colleagues' (1979) expansion of the two-factor theory proposed that the post-traumatic stress symptoms developed through classical conditioning are then sustained and promoted through avoidance, which is learned via operant conditioning - and specifically negative reinforcement: When individuals are presented with a trigger, they engage in avoidance, which temporarily removes the trigger; this results in immediate relief, reinforcing the pattern of avoidance.

The foundation laid by Kilpatrick et al. (1979) has remained largely unaltered in subsequent conditioning-based discussions of the acquisition and maintenance of PTSD (e.g., Keane, Zimering, \& Caddell, 1985; Kilpatrick, Veronen, \& Best, 1985). Recent expansions of the two-factor conditioning model have focused on deficits in the ability to extinguish fear responses. Models of conditioned fear response have been the basis of exposure-based treatments for phobias and other anxiety disorders, as well as PTSD. Theoretically, if stimuli can be conditioned to produce a fear response, the same stimuli can be reconditioned to produce a neutral response, thus extinguishing the fear response, or leading to fear extinction (Gillihan et al., 2014). That repeated exposure to anxiety- 
causing stimuli can decrease an individual's emotional response to them is welldocumented in the literature. People with PTSD, however, show significant deficits in fear extinction (Lissek et al., 2009). Research has also found that people with PTSD have difficulty recalling fear extinction responses (neutral responses to conditioned stimuli) in some circumstances even after they have demonstrated this learned extinction response in other circumstances. Milad and colleagues $(2008,2009)$ proposed this deficit is in recall of the learned extinction response. According to this theory, individuals with deficits in learning or recalling extinction responses may come to rely on avoidance techniques to manage their fear response, so avoidance and fear extinction deficits work in tandem to maintain and promote PTSD symptoms (Gillihan et al., 2014).

Using conditioning to understand PTSD development has its shortcomings. This model is an extension of general anxiety models, and as Gillihan and colleagues (2014) pointed out in their critique, does not explain empirical evidence that shows differences between phobias and PTSD (e.g., increased generalization of stimuli, poorer response to treatment). Additionally, it is unclear from these models and current research whether difficulty with fear extinction deficits are due to pre-existing psychological vulnerabilities or result from the trauma experience itself. Gillihan and colleagues (2014) also argued that while deficits in fear extinction explain subsequent and chronic triggering experiences in individuals with PTSD, they need to be reconciled with fairly robust literature demonstrating the efficacy of exposure-based treatments in reducing PTSD symptoms. Finally, these models do not address the increasing body of literature supporting the DSM-5's expanded conceptualization of PTSD as more than an anxiety disorder, which includes symptoms beyond those explained by fear response. 
Still, conditioning theories are helpful in understanding the fear-based PTSD symptoms transgender people experience in response to bias events (Reisner et al., 2016). Trauma responses to seemingly non-threatening bias-based stimuli could be characterized as generalizations of conditioned responses, if a person has previously been exposed to similar bias events in the context of threatening situations (as is a frequent experience for many transgender people; e.g., Grant et al., 2011; Shipherd et al., 2014). Additionally, if a key part of the reduction of post-traumatic stress is the learning and recall of fear extinction responses (Lissek et al., 2009), the frequency of bias-based threatening situations alone would interfere with transgender people's recovery and thus encourage further generalization of conditioned responses.

Emotional Processing Theory. Emotional Processing Theory (Foa \& Cahill, 2001; Foa \& Kozak, 1985, 1986) is an etiological theory of PTSD that shares similarities with conditioning models, but can account for non-fear-based cognitions, emotions, and other symptoms. Foa and Cahill's (2001) model of PTSD posits that the symptoms of PTSD represent activations of maladaptive emotion structures that were developed during the trauma experience and then maintained and expanded through subsequent activations. According to this theory, emotion structures are memory structures that "include emotion-related stimuli, responses, the meanings associated with these stimuli and responses, and the associations among these representations" (Gillihan et al., 2014 p. 169. These are typically adaptive and include scripts for appropriate (and sometimes lifesaving) actions. Scripts are a type of schema structure that include behavioral and social responses to stimuli (Foa \& Cahill, 2001). Maladaptive structures develop due to inaccurate associations between responses, or understandings of stimuli that do not reflect reality. Emotional Processing Theory suggests that there are two principal components to 
the maladaptive emotion structures underlying PTSD: a fear structure that has generalized to include non-threatening stimuli; and an emotion structure that includes the individual's response to the trauma and a sense of incompetence in protecting themselves from harm. They described these structures as leading to "two broad sets of negative cognitions: 'the world is entirely dangerous' and 'I am completely incompetent"” (Gillihan et al., 2014, p. 170). The emotion structure can include the feelings of shame that are also characteristic of many cases of PTSD, and which some psychologists proposed are an integral piece of the development and maintenance of post-traumatic stress (e.g., Lee et al., 2011).

In addition to providing a broader understanding of the etiology of post-traumatic stress as a complex response to stimuli, emotional processing theory offers an explanation for the natural recovery often seen in survivors of trauma. Foa and Kozak (1986; Foa \& Cahill, 2001) explained that emotional processing of trauma allows individuals to recover from the trauma without developing PTSD. This recovery occurs because in each experience of processing, survivors are exposed to evidence that disconfirms their negative cognitions about the world and themselves. According to this theory, PTSD develops when trauma survivors fail to process their traumatic memories adequately and instead strengthen their maladaptive emotion structures. Thus, beyond conceptualizing avoidance as a source of negative reinforcement for trauma responses, emotional processing theory views avoidance as an obstacle to the processing that is necessary for dismantling of maladaptive of emotion structures (Foa \& Cahill, 2001). There is empirical evidence to support this theory. Gilboa-Schetman and Foa (2001) found that trauma survivors whose distress peaked three weeks or more after the trauma suffered from more severe PTSD symptoms months later when compared to survivors 
whose distress peaked within the first two weeks of the trauma. Foa and colleagues (2007) developed prolonged exposure therapy (PE) to help clients with PTSD process their trauma memories and engage with evidence that disconfirms their beliefs about threats and their ability to handle them. PE has demonstrated considerable efficacy as a treatment (Powers, Halpern, Ferenschak, Gillihan, \& Foa, 2010), lending strong empirical support to emotional processing theory. This work has considerably advanced the field's understanding of trauma and trauma treatment (Gillihan et al., 2014), and provides a helpful framework for conceptualizing post-traumatic stress development in transgender people. It is particularly useful in its inclusion of non-fear-based cognitions and emotions within emotional structures that can be triggered by various stimuli. An emotion structure that has been strengthened by an anti-transgender trauma, for example, may include a bias event as a trigger, internalized transphobic cognitions and shame as internal responses, and certain PTSD symptoms and behavioral responses.

The limitations of emotional processing theory revolve around its reliance on a clearly defined and singular trauma event. Additionally, Foa and colleagues' concept of recovery from trauma and PTSD rests on a major assumption that survivors' appraisals of the world and themselves are inaccurate and can be disconfirmed. These represent major limitations in the theory's application to the traumatic experiences of transgender people, which are chronic in nature (Mizock \& Lewis, 2008; Shipherd et al., 2014), and which stem from a climate that truly is unsafe for many transgender people (Grant et al., 2011).

Additionally, although PE is one of the strongest evidence-based treatments available to individuals with PTSD, a substantial minority of those who receive treatment do not improve, and PE and other exposure treatments struggle with poor adherence and drop-out rates (Powers et al., 2010). The high rates of drop-out and poor adherence 
suggest that there are individuals whose trauma has affected them in ways beyond the scope of this theory (Herman, 1992).

Schema theories. Schema theories of PTSD originated from psychoanalytic and information processing schools of psychology (Gillihan et al., 2014). These psychological theories conceptualize PTSD as resulting from the cognitive dissonance and schema work that occurs following a trauma. Horowitz (1986) first proposed this approach to PTSD, arguing that traumatic events contradict survivors' beliefs about others, the world around them, and/or themselves; he argued that this contradiction, or schema incongruence, itself was traumatic as a form of severe cognitive dissonance. Trauma theorists from this school specifically argue that traumas violate the following belief schemata: the world is benign and meaningful, the self is worthy, and people are trustworthy (Epstein, 1991). Horowitz (1986) argued that recovery from trauma involved making sense of the traumas by adapting the information about the trauma and one's core schemas until they align with each other. This work borrowed heavily from Piaget's long accepted theories about the use of cognitive assimilation and accommodation to relieve disequilibrium (Gillihan et al., 2014). Intrusion symptoms are the primary result of the dissonance, though avoidance symptoms may also arise from this schema incongruence (Horowitz, 1986). McCann and Pearlman's (1990) schema-based conceptualization of trauma included many of Horowitz's original ideas, but also highlighted trauma-related strengthening of pre-existing maladaptive schemas as an additional source of PTSD symptomology. They suggested that it was not just the experience of cognitive dissonance that was traumatizing, but that in attempting to make meaning out of a trauma, a survivor could reinforce unhelpful and unhealthy ideas about themselves and the world (e.g., that the world is unsafe, or that the self is devalued). In this instance, 
schema congruence would be the mechanism behind PTSD development, particularly in negative alterations in mood and alterations in arousal.

Gillihan and colleagues (2014) explained schema theories as sources of advancement in the field's understanding of how trauma affects the way survivors make sense of the world and themselves. Trauma literature now readily recognizes that traumatic events can have profound impact on a person's beliefs about the world (Gillihan et al., 2014). Indeed, one might note that Foa \& Cahill's (2001) 'emotion structures' are similar to the concept of schemas. Feminist trauma psychologists, psychologists studying racist-based trauma, and psychologists studying shame-based trauma also draw upon the tenets of Horowitz's (1986) and McCann and Pearlman's (1990) trauma theories. The theories' strengths for these psychologists are in their emphases on the mental model of the event as the source of trauma. Schema theory, however, has significant gaps in explanatory power, most prominently that it is unclear when scheme incongruence is a risk factor for post-traumatic stress and when scheme congruence is a risk factor (Gillihan et al., 2014). Gillihan and colleagues (2014) are also critical of the theory for its difficulty to operationalize and test.

Feminist conceptualizations and etiological theories. Feminist understandings of trauma and its impact rely upon key assumptions that run counter to the mainstream psychological approach to trauma and post-traumatic stress. Paramount among feminist assumptions of trauma is that "what is deemed traumatic is determined by the traumatized person rather than the observer" (Root, 1992, p. 230). By emphasizing the psychosocial context of individuals and the traumas they experience, feminist theories of trauma define trauma according to the impact an event has on a person, rather than solely by characteristics of the event itself. This perspective is a piece of the challenge to the 
aforementioned debate about defining trauma events in Criterion A of the DSM diagnostic criteria for PTSD. The feminist theories discussed in this section appear to place little importance on strictly defining trauma and the sequelae following traumata, again challenging the approach taken by the DSM- 5 and more mainstream trauma psychologists. Although aforementioned mainstream trauma psychologists such as Foa and Resick developed their theories and treatments with the stated purpose of creating measurable constructs (Resick et al., 2012), most feminist trauma psychologists explicitly state they are not concerned with developing or presenting a theory constructed for empirical testing, instead focusing on the at-times opposite goal of broadening our understanding of trauma and placing it within the context of human psychology in general (Root, 1992).

Root's feminist reconstruction of the impact of trauma. Maria Root's (1992) seminal work on a feminist conceptualization of trauma, Reconstructing the Impact of Trauma on Personality, provided an alternative framework for understanding trauma. Her work draws from a transtheoretical core component of definitions of the impact of trauma: disorganization of the world and self. Citing emotional processing theory (Foa et al., 1989) and schema theory (Horowitz, 1976; Janoff-Bulman,1985), she described how traumatized individuals experience "a shattering or destruction of different constellations of organizing principles by which we come to know ourselves, others, and the world" (p. 251). Root (1992) referred to these constellations of organizing principles as dimensions of security and theorizes that these security dimensions are physical, psychological, or interpersonal (see Table 2 for her description of some of the ways traumata affect these dimensions). Root suggested that every instance of destruction of an aspect of a dimension of security is a trauma, as it represents a sudden and dramatic disorganization. 
Thus, a traumatic event is actually itself a constellation of multiple trauma experiences, the specific signature being determined by details of the traumatic event, the context of the event, and the person's history and identity.

Table 2

Root's (1992) Dimensions of Security

\begin{tabular}{|c|c|}
\hline Dimension & Explanation \\
\hline \multicolumn{2}{|l|}{ Physical } \\
\hline$\overline{\text { Stimulus deprivation }}$ & $\begin{array}{l}\text { Lack of stimulation, particularly early in life, may } \\
\text { permanently alter or retard CNS development; stimulus } \\
\text { input also provides a grounding in reality; SD includes } \\
\text { restricted movement; confinement }\end{array}$ \\
\hline Pain & $\begin{array}{l}\text { Immediate CNS response, severe pain likely to leave } \\
\text { 'memory traces' }\end{array}$ \\
\hline Injury & $\begin{array}{l}\text { Almost always involves pain and may also be } \\
\text { accompanied by visual cues (e.g., blood), auditory cues } \\
\text { (e.g., screams, gunfire) }\end{array}$ \\
\hline Permanent injury & $\begin{array}{l}\text { Leaves indelible visual, functional, and/or kinesthetic } \\
\text { reminder of trauma }\end{array}$ \\
\hline Starvation & $\begin{array}{l}\text { Also related to pain and associated with survival } \\
\text { behaviors: hoarding, dreams about food, hallucinations, } \\
\text { antisocial behaviors to obtain food }\end{array}$ \\
\hline \multicolumn{2}{|l|}{ Psychological/spiritual } \\
\hline Confrontation with mortality & Confront reality of fragility of life and reality of death \\
\hline Loss of significant other(s) & Impairs sense of belonging, existence, identity, security \\
\hline Perceived malicious intent & $\begin{array}{l}\text { Raises fear, anticipation of harm; injure belief in } \\
\text { benevolent action }\end{array}$ \\
\hline Isolation & $\begin{array}{l}\text { Alienation, lack of opportunity to test reality or assign } \\
\text { appropriate cause/responsibility; contributes to feeling } \\
\text { helpless and constructing idiosyncratic meaning }\end{array}$ \\
\hline Helplessness/loss of control & Resignation, cessation of hope \\
\hline $\begin{array}{l}\text { Witness/participant to death } \\
\text { or destruction }\end{array}$ & Human life not valued; right and wrong nonapplicable \\
\hline Crushing of spirit & $\begin{array}{l}\text { Emotional abuse, brainwashing, and destruction result in } \\
\text { humiliation, perceived lack of meaning to one's } \\
\text { existences, lack of zest for life }\end{array}$ \\
\hline Dislocation & $\begin{array}{l}\text { Such as in refugee experience; loss of identity, } \\
\text { homebase, country, culture, attachment - results in } \\
\text { disorientation and being ungrounded }\end{array}$ \\
\hline \multicolumn{2}{|l|}{ Interpersonal } \\
\hline Betrayal & $\begin{array}{l}\text { Devastates trust and willingness to be interpersonally } \\
\text { vulnerable }\end{array}$ \\
\hline Abuse of power & $\begin{array}{l}\text { Fails to provide social order and assurance of safety; } \\
\text { impairs trust }\end{array}$ \\
\hline Violation of personal space & $\begin{array}{l}\text { Damages sense of control over space; alters notion of } \\
\text { inter- and intrapersonal boundaries }\end{array}$ \\
\hline
\end{tabular}


Rejection

Invisibility

Loss of significant other(s)
Devalues worth; negates existence; may contribute to deprivation in children

Ignores existence, devalues worth, sentence of "death" Impairs sense of belonging, intimacy, trust

Note. Table from Root (1992, pp. 252-253)

This reconstruction not only captures the multitude of ways that violence or threat of violence can affect a person, it also provides a framework for understanding how nonphysically threatening experiences can still be injurious in traumatic ways. For example, the chronic experience of anti-transgender discrimination could be conceptualized using Root's (1992) security dimensions as destructive of psychological and interpersonal dimensions, resulting in "fear and anticipation of harm," "perceived lack of meaning in one's existence," "devastat[ion] of trust and willingness to be personally vulnerable," “devalue[d] worth," and "impair[ed] sense of belonging," (Root, 1992, pp. 252-253).

Root referred to this type of non-physical, oppression-based trauma as insidious trauma. Insidious traumata include daily reminders to a person that their status is devalued in society due to an intrinsic characteristic (or group membership) that differs from the norms or values of those with more social power. In addition to insidious trauma, Root defined two other categories of trauma: direct and indirect. Her concept of direct trauma is the category most aligned with the mainstream definitions of trauma, though Root's also included life-threatening illness, emotional abuse, and violence perpetrated against a community to which one belongs. For the latter, she offers an example of the trauma an indigenous person might experience due to their ancestors being dislocated and/or victims of genocide. At the time of Root's work, indirect exposure to trauma was not considered by mainstream psychology to be traumatic in and of itself; this has since changed. The current Criterion A includes indirect exposure to a close family member or close friend's trauma. Root's concept of indirect trauma includes 
being traumatized by trauma experienced by anyone "with whom one identifies in some significant way" (p. 239), thus expanding definitions of indirect trauma to include community members and those with similar identities, as well as therapists and others in helping positions who may be traumatized by the trauma sustained by their clients. Notably, this level of indirect traumatization is more and more accessible, as social media connections has intensified the ways in which people are exposed to community members' trauma.

Building upon schema theory, Root posited that the sequelae of trauma are the manifestation of an individual's efforts to reorganize their security dimensions following traumata. Although many individuals who experience trauma re-establish a sense of themselves and a sense of the world that are not terribly different from what existed pretrauma, many survivors do not. Root (1992) explained many of the symptoms and behaviors associated with PTSD, as well as other trauma-related disorders such as borderline personality disorder, histrionic personality disorder, and depression, as evidence of reorganization that is "developmentally idiosyncratic and survival-oriented" (p. 255). According to Root, much of what is seen in trauma survivors' presentations can be described within a framework of stages of vigilance and survival behaviors. Drawing from neurobiological evidence and decades of cognitive theory and research, Root explained that response to threatening cues occurs on three sequential levels or stages: readiness, alert, and survival. Readiness describes an ability to detect threatening sensory experiences, events, and people; it involves scanning behaviors, constant employ of working memory, and weak potentiation, meaning individuals in a readiness level are quicker to react to stimuli. Alert is a level of response to threatening cues, in which one's energy and resources are directed toward the threat, but the individual is aware of their 
heightened level of vigilance and is still able to engage in some metacognition to evaluate validity of the threat. Survival is the level of response in which an individual can no longer assess for the validity of the threat and as such responds to all possible threats as though they are truly threatening. The individual's "perceptual, decisional, and relational process are transformed" by the redirection of all energy and focus to threat response (Root, 1992, p. 247).

Root argued that the destruction of dimensions of security leave a trauma survivor in a constant state of readiness. The literature since Root produced this theory has supported her conclusions: trauma survivors displaying PTSD symptoms consistently show neuroendocrine and even structural brain abnormalities that lead to increased reactivity, near-constant threat monitoring, cognitive deficits associated with reallocation of resources, and difficulty with memory and present/past separation (e.g., Yehuda \& LeDoux, 2007). Because of this heightened level of readiness and reactivity, as well as reorganization that may have prioritized survival, traumatized individuals are often operating from a survival mode. Root argued that many of the behaviors the field of psychology has at best labeled impairments or maladaptive functioning, and at worse described as regressive or unstable, are truly self-preservation behaviors in individuals who are understandably experiencing a near-constant state of threat. Much of the sequelae associated with post-traumatic stress disorder and trauma-related personality disorders can be described within Root's categories of survival behaviors: selfreferencing behavior; egocentrism; preservation; anger; withdrawal and shutting down; and splitting. Recovery, be it 'natural' or treatment-aided, from post-traumatic distress thus occurs when an individual can reorganize their dimensions of security to better modulate their stages of vigilance, enact non-survival behavioral responses to possible 
threats, and engage in flexibility around when to employ survival behaviors. Root argues that this is mostly possible only in the context of absence of trauma, but this is a necessary and not sufficient component of recovery.

Insidious trauma may be particularly impactful in the development of idiosyncratic and survival-based security dimensions. Root (1992) and other theorists proposed that the traumatic effects of insidious trauma (chronic and sometimes subtle by their nature) may be the result of their role in gradual shaping views of the world and self, rather than the "shattering" of such schema or security dimensions that is attributed to single event traumas (Brown, 2008; Richmond et al., 2012). Additionally, the chronic level of threat inherent in insidious trauma prevents the development of non-survival behaviors and psychological flexibility required for recovery (Root, 1992).

To provide further understanding as to why some trauma experiences are more likely lead to sustained disorganization or heightened levels of vigilance, Root (1992) also proposed a two-dimensional phenomenological categorization of traumatic events: by perceived intent (malicious or accidental) and perceived interpersonal context (in isolation or with companion(s)). The word "perceived" is important to the feminist framework Root is presenting, as "the intrinsic meaning of the event must be determined by the person experiencing it, rather than the observer" (Root, 1992, p. 243).

Understanding where a trauma falls on these dimensions helps one understand how the trauma might affect certain security dimensions and subsequent reorganization. Most importantly, the more the intent behind a trauma is perceived to be malicious and the more the trauma survivor perceives themselves to be isolated, the more likely the survivor and others are to understand the trauma experience through a lens of unique vulnerability (Perloff, 1983, as cited in Root, 1992). This concept is akin to victim- 
blaming and self-blaming (Crawford, 1977; George \& Martinez, 2002; Janoff-Bulman, 1979). Unique vulnerability means that there is or was something about the survivor that made them fall victim to the traumatic event. Experiencing repeated or chronic traumas can reinforce a sense of unique vulnerability, both to external observers and to the survivors themselves. Root argues that a perception of unique vulnerability prevents trauma survivors from reconstructing their lives, seeking and receiving support, and even identifying the event as traumatic. This lack of progress occurs in part due to increased experiences of shame in survivors who have a sense of unique vulnerability (Brown, 2008). Unique vulnerability also leads to an increased sense of persistent threat and reorganization that is necessarily survival-based. This might help explain the differential rates of persistent post-traumatic stress for events like rape versus natural disasters (Kessler, Sonnega, Bromet, Hughes, \& Nelson, 1995); rape victims are more likely to be viewed as somehow contributing to their trauma (Brown, 2008), and may experience the consequences of unique vulnerability that Root (1992) theorized.

Insidious traumata can be particularly devastating to the dimensions of perceived intent and perceived interpersonal context, as well as to the perception of a unique sense of vulnerability. Although insidious trauma can often be attributed to membership to a stigmatized/oppressed group, creating a sense of individual-level companionship in the trauma, Root (1992) described a concurrent experience of group-level isolation/alienation from mainstream society. The latter reduces the amount of support and resources available to survivors of insidious traumata. Additionally the targeting of an individual due to group membership can reinforce a complex sense of unique vulnerability. When trauma is perceived to be due to bias against an individual characteristic, it can be easy for others and the victim to hold the victim responsible (Brown, 2008). Because violence 
and other anti-transgender bias are more likely when a transgender person presents as their authentic gender identity (e.g., a transgender woman who was male assigned at birth presenting in feminine clothing; Grant et al., 2011; Shipherd et al., 2014), transgender victims of anti-transgender bias-based trauma may be particularly susceptible to outsider projections and internalizations of unique vulnerability and accompanying shame (Brown, 2008). The chronic nature of insidious trauma further contributes to a sense of unique vulnerability (Comstock, 1989; Parson, 1985; Root, 1992).

Root's conceptualization of insidious trauma has had the largest impact on the field of trauma psychology. Her work has been the foundation for theory and research on racist incident-based trauma, homophobic/heterosexist incident-based trauma, and conceptualizing anti-transgender bias events as trauma (Bryant-Davis \& Ocampo, 2005; Richmond et al., 2012; Szymanski \& Balsam, 2011). In the past two decades, psychologists looking at the impact of racism and the oppression of sexuality minorities (people who identify their sexuality as something other than heterosexual or straight) have drawn upon and expanded insidious trauma theory to explain increased rates of PTSD and trauma-related distress and diagnoses in minority communities. A large body of research has found that within the United States, people of color are more likely than White people to meet diagnostic criteria for PTSD (e.g., Roberts, Gilman, Breslau, Breslau, \& Koenen, 2011). When compared to White people, people of color are more likely to develop PTSD in the aftermath of Criterion A PTEs, even those (e.g., natural disasters) that would be considered completely unrelated to a person's racial and ethnic identities (e.g., Breslau, Cilcoat, Kessler, \& Davis, 1999; Perilla, Norris, \& Lavizzio, 2002). Additionally, being victimized by a hate crime (a crime in which a person's identity or identities are determined to be a motivating factor) is associated with greater 
risk for PTSD (Herek et al., 1999). Psychologists have explained some of these findings by treating minority status as a risk factor due to the increased likelihood of acculturative stress, which may reduce a person's ability to effectively cope with traumas (Kulkarmni $\&$ Pole, 2008). Others challenge this as the sole explanation and argue that racism and ethnoviolence are themselves insidious traumata and the actual sources of symptoms and behaviors associated with PTSD (Bryant-Davis \& Ocampo, 2005; Helms, Nicolas, \& Green, 2010; Loo et al., 2001). Research has supported this application of the concept of insidious trauma. Individuals who have more exposure to racist and ethnicity-based bias are more likely to experience PTSD when compared to individuals with less exposure to these biases, even after accounting for the effects of Criterion A PTEs (e.g., Chou, Asnaani, \& Hofmann, 2012; Ellis, MacDonald, Lincoln, \& Cabral, 2008). Paradies and colleagues' (2015) meta-analysis, which included 16 studies that examined the relationship between racism and post-traumatic stress symptoms, found an effect size of $r$ $=.34$ for racism on PSTD symptoms and diagnoses. Similar relationships with PTSD have been found with anti-woman bias experiences and sexual harassment (e.g., Eillness, Steel, \& Lee, 2007; Kira, Smith, Lewandowski, \& Templin, 2010), as well as anti-LGBQ bias experiences (e.g., Alessi, Martin, Akua, Gyamerha, \& Meyer, 2013; Szymanski \& Balsam, 2011). The limited research that has been done on transgender people's experiences of bias and PTSD have also supported Root's (1992) theory of insidious trauma. Reisner and colleagues (2016) found that transgender people who experienced more discrimination were more likely to have PTSD symptoms, even after controlling for exposure to Criterion A potentially traumatic events (PTEs).

Complex PTSD. In the same year that Root introduced her reconstruction of trauma, Herman (1992) proposed her own alternative framework for understanding the 
impact of interpersonal trauma: complex PTSD. Richmond and colleagues (2012) largely based their trauma framework for transgender mental health in this particular trauma theory. Herman (1992) critiqued the existing DSM criteria for defining trauma solely as single events and failing to capture the myriad ways that trauma can affect survivors, particularly when it is interpersonal, chronic, and cumulative. She proposed reconceptualizing post-traumatic stress disorders as existing on a continuum that ranges from the classic simple PTSD described in the DSM to complex PTSD, which develops in response to prolonged or repeated interpersonal trauma. By the nature of the trauma being chronic, survivors of repeated trauma are "in a state captivity, unable to flee, and under control of the perpetrator" (Herman, 1992, p. 377). Herman argued that this captivity and the related interpersonal dynamics and persistent threat to safety complicate the trauma experience and lead to a host of symptomatic and characterological trauma sequelae. The hypervigilance that is learned as a protective strategy during trauma extends beyond traumatic situations, and individuals with complex PTSD often present as anxious and constantly agitated at baseline, often without a perception of ever achieving calm; this leads to a host of somatization difficulties, which is supported by research that finds increased chronic physical health problems in survivors of complex trauma. Dissociation, which is often employed voluntarily as a tool to cope with trauma, becomes a process over which the survivor loses control. Herman (1992) cited research and theory that describes traditional dissociation symptoms in complex trauma survivors, as well as mind fragmentation and disturbances in memory, concentration, and sense of time. Other symptomatic sequelae stem from the harsh violation of the survivor's belief systems. Survivors of complex trauma have a sense of being "forsaken by man and God" and suffer from a "debased self-image" (p. 382), leading to severe depressive symptoms. 
Rage is often experienced, but due to coping strategies that helped the survivor tolerate their physical or psychological captivity, they may identify with or have sympathy for the perpetrator(s). Rather than recognizing their rage at those who inflicted the trauma upon them, survivors of complex trauma may direct their rage inward, and develop self-hatred and chronic suicidality (Herman, 1992). This complex array of symptomatic changes is presented by Herman as evidence that PTSD diagnostic criteria are too limited; she cites high comorbidity rates with mood and anxiety disorders to highlight the limitations of DSM criteria. Epidemiological research shows that high rates of comorbidity persist (e.g., Campbell et al., 2007; Kessler, Sonnega, Bromet, Huges, \& Nelson, 1995).

Herman (1992) described the characterological sequelae as pathological changes to relationships and identity. This is where Herman most clearly diverges from mainstream trauma theory; she describes relationship and identity difficulties that are currently discussed in DSM nosology only with regard to personality disorders. And indeed, people with personality diagnoses are more likely than the general population to have experienced complex trauma (Zimmerman \& Mattia, 1999). Herman (1992) explicitly stated her concern with this "misapplication of the concept of personality disorder," writing that "concepts of personality developed in ordinary circumstance are frequently applied to survivors, without an understanding of the deformations of personality which occur under conditions of coercive control" (p. 388). Herman proposed a number of ways through which ultimately unhealthy relationship or interpersonal behaviors can develop in direct response to trauma. Namely, because the chronic trauma occurred in an interpersonal context, in which the survivor was gradually disempowered, individuals with PTSD develop an intense need for attachment and an intense fear of attachment. Herman (1992) also theorizes that the severe identity disturbances seen in 
survivors of complex PTSD are developed in direct response to the chronic trauma. She argued that the coercive control at the heart of her conceptualization of complex trauma systematically breaks down a victim's structures of the self, resulting in a loss of a sense of one's own humanity and/or a coherent sense of purpose. Many survivors view themselves as guilty, evil, or inherently wrong; this may be coped with by utilizing splitting strategies and identity fragmentation.

A substantial body of work has focused on testing and advancing Herman's (1992) conceptualization of complex PTSD and a spectrum or dimensional approach to post-traumatic stress reactions. Briere and Spinazzola (2005) reviewed this and similar literature and proposed the following categories of complex PTSD phenomenology: altered self-capacities, cognitive disturbance, mood disturbance, overdeveloped avoidance responses (manifested in dissociation, substance abuse, and tension reduction by way of avoidant coping), somatoform distress, and traditional posttraumatic stress (i.e., the DSM-IV-TR symptoms clusters of avoidance, hyperarousal, and intrusion). Beyond recognizing the phenomenology as distinct from classic PTSD, research has also supported differentiation between simple and complex PTSD with regard to response to treatment. The prognosis for individuals with complex PTSD is bleaker than for individuals with classic PTSD; treatment outcomes are poorer and dropout rates are high (Foa, Keane, \& Friedman, 2000; Spinazzola, Blaustein, \& Van der Kolk, 2005). Research has also found that for individuals with complex PTSD, treatment outcomes are predicted by the quality of the therapeutic relationship (Pearlman \& Courtois, 2005), highlighting the interpersonal nature of their trauma and its impact. The phenomenon of complex PTSD is commonly accepted by clinicians, and a multitude of leaders in the field of trauma psychology have called for a complex PTSD diagnosis to be included in the 
DSM. Most recently the committee tasked with reviewing diagnostic updates related to trauma in the fifth edition of the DSM reviewed a proposal for a complex PTSD diagnosis, as well as a proposal for a spectrum approach to post-traumatic stress disorders. They found the evidence for differentiating was not compelling enough to introduce an additional trauma-related diagnosis into the DSM-5 (Resick et al., 2012), namely because the constructs of complex trauma and complex PTSD were looselydefined and could not be operationalized efficiently enough to establish divergent validity from the existing PTSD diagnosis.

Applying a complex PTSD framework to transgender systems of trauma is beneficial because complex PTSD symptomology is much more expansive than traditional DSM PTSD criteria. With complex PTSD as a guiding framework, much of the disparities in transgender psychopathology can be understood as trauma sequelae (Richmond et al., 2012). Depressive symptoms, suicidality, self-harm behavior, shame, interpersonal difficulties, maladaptive personality traits can arise as responses to chronic interpersonal trauma (Briere \& Spinazzola, 2005; Herman, 1992).

The theory's primary limitation for our purposes is that complex PTSD did not originally present insidious trauma as a possible source of complex PTSD sequelae. Herman's (1992) formulation discussed abuse situations that included battered women, abused children, prisoners, and cult members. The commonality between the survivors and types of trauma she referenced was that the trauma occurred in a state of "subordination to coercive control" (Herman, 1992, p. 378), but she did not explicitly envision oppression (the context of insidious trauma) as sharing this characteristic. Other theorists, however, have expanded the boundaries of complex trauma to include insidious traumata and closed this gap (e.g., Brown, 2008). Notably, psychologists whose work 
focuses on racism as traumatic have demonstrated parallels between racist incidents and the interpersonal traumas that lead to complex PTSD (Bryant-Davis \& Ocampo, 2005).

Racist-incident based trauma. In their conceptualization of racist incidents as trauma, Bryant-Davis and Ocampo (2005) utilized McFarlane and Giorlama's (1996) definition of traumatic stress: distress which “violates one's existing way of making sense of self and the world" and thus leads to destabilization and/or fear (p. 485). They argued that racist incidents are traumatizing when they have this destabilizing effect and attack a person's selfhood. They supported their argument with a careful, critical examination of the ways in which racist incident-based trauma parallels both rape- and domestic violence-based trauma, using empirical evidence to support their conclusions. BryantDavis and Ocampo's work was built upon that of Carter and Helms (2002) and Wyatt (1990) who noted similarities between the effects of childhood sexual abuse and racism: "feelings of shock, betrayal, and powerlessness, and the sense of being stigmatized as 'not good enough"” (Bryant-Davis \& Ocampo, 2005, pp. 486-487), as well as previously mentioned theorists and researchers who first drew attention to the traumatic nature of racism (e.g., Loo et al., 2001).

An important parallel between domestic violence and racist incidents is the complex duality of coming to expect the trauma but experiencing shock and disorganization when experiencing it.

Survivors living with domestic violence live with the expectation that a violation may occur, although they are unaware of the form the violation will take (Jacobson \& Gottmann, 1998). Similarly, many target group members who live with racism live with the expectation that racism will be felt, yet they are unsure of when the incident will 
occur or of what type of racism they will face. Knowing neither what will happen nor how devastating the effects will be contributes to hyperarousal and anxiety. This marriage of expectancy and shock is unique to experiences of trauma such as domestic violence and racism. (Bryant-Davis \& Ocampo, 2005, p. 492)

Bryant-Davis and Ocampo explained that in addition to this hyperarousal and anxiety, victims experience internalized blame, as survivors of both domestic violence and racist incidents are told they could avoid these traumatic events if they behaved in certain ways. This internalized sense of responsibility (akin to Root's unique vulnerability terminology) leads to a sense of powerlessness, as victims recognize that even when they "attempt to follow the numerous and often arbitrary rules" (Bryant-Davis \& Ocampo, 2005, p. 492), they continue to be violated. In their exploration of the parallel with rape survivors, Bryant-Davis and Ocampo noted that sexual assault survivors are more likely to blame themselves for their trauma if they have internalized negative beliefs about their gender or sexuality; similarly they cited Sellers and Shelton's (2003) finding that African-Americans who held negative beliefs about African-Americans were less able to cope with racist discrimination. Another parallel between sexual assault trauma and racist incident trauma is the possibility of secondary trauma due to identification with a target group. Awareness of potential victimization can lead to hypervigilance and related symptomatology among individuals who have not yet been directly targeted (Baranowsky, Johnson-Douglas, Williams-Keeler, \& McCarrey, 1998). Finally, Bryant-Davis and Ocampo (2005) noted that survivors of all three traumas struggled with trust and relationships. 
But neither parallel fits perfectly, which Bryant-Davis and Ocampo pointedly stated. The parallel with rape works best when looking at survivors of chronic sexual assault, as the chronicity of racist incidents is critical in understanding its impact on individuals. And yet chronic sexual assault and domestic violence are typically perpetrated by consistent parties, typically a single person, whom the victim knows. The accumulation of racist incidents is comprised of multiple perpetrators, many (if not the majority) of whom the survivor does not know. And as Bryant-Davis and Ocampo (2005) noted, a key piece of the trauma of racist-based incidents involves the attack on a person's sense of self that results from the knowledge or perception that the trauma occurred due to one's identity. This attack on sense of self (as opposed to an attack on an individual's physical self) extends beyond mainstream etiological models of posttraumatic stress and does not fit easily into parallels with other traumas.

The work on racist incident-based trauma obviously lends a great deal to an understanding of anti-transgender bias-based trauma, as both are rooted in identity-based oppression (Richmond et al., 2012). However, experiences of anti-transgender bias differ from experiences of racism in meaningful ways. Though both represent attacks on the self in terms of one's understanding of their worth and social value (Bryant-Davis \& Ocampo, 2005; Richmond et al., 2012), anti-transgender bias typically also involves an attack on the actual validity of the transgender person's gender identity. Transgender people's identities are routinely denied, either explicitly or via the requisite of external validation of a transgender person's claimed gender identity. Many transgender people's coming out experiences involve having to defend the legitimacy of their identities (Bockting \& Coleman, 2016). Transgender people are routinely told directly or exposed indirectly to messages that transgender people's gender identities are not real (Mock, 
2014). Even among supportive medical and mental health professionals, many explicitly require some level of assessment of a transgender person's gender identity and/or mental stability before providing transition-related care (e.g., Khan, 2011). The United States government issued a policy in 2013 which requires that transgender people provide a letter from a physician certifying their gender identity and expression before they can apply for an accurate passport (U.S. Department of State, undated); the gender options on the passport are still limited to male and female, which excludes nonbinary people from having an accurate gender marker. Many states require proof of surgical operations before transgender people can update their identification documents (National Center for Transgender Equality, 2016), and these again are limited to male and female. Rather than discussing the political nature of these policies, nor debating their practical merit, these facts are presented to highlight that transgender people's daily lives involve some degree of consistent challenging of their gender identities and their ability to define such identities for themselves. If transgender people interpret anti-transgender bias as attacks not only attacks on their worth but also on their very definition of themselves, they may be experiencing particularly severe attacks on their sense of self. Recognizing that traumatization involves the violation of "one's existing way of making sense of self and the world and creates intense fear and destabilization" (McFarlane \& Giorlama, 1996, p. 485), anti-transgender bias-based insidious trauma may be particularly traumatic in ways beyond what was captured in Bryant-Davis and Ocampo's (2005) work.

Integrative summary. In this section, I have reviewed research on transgender people's risk for negative mental health outcomes, as well as their exposure to antitransgender bias, and the adaptations of the Minority Stress Model that propose ways in which these are related. I have proposed following Richmond and colleagues' (2012) 
trauma framework as a better way of understanding transgender experiences by defining anti-transgender bias experiences as potentially traumatic and conceptualizing the documented disparities in mental health and psychological/interpersonal functioning as representative of trauma sequelae. To do this, I have drawn upon trauma theory from mainstream sources (DSM-5; conditioning theory; emotional processing theory) and feminist and multicultural sources (Root's, 1992, reconstruction of trauma theory, including insidious trauma; complex PTSD; and racist incident-based trauma theory) and contextualized these within a history of trauma psychology that has routinely invalidated the experiences of marginalized survivors in its attempts to define trauma and trauma sequelae.

\section{The Role of Shame and Internalized Stigma in Trauma}

I have already briefly discussed shame as a piece of trauma sequelae in my review of modern understandings of trauma and post-traumatic stress. As I propose internalized transphobia is a key factor in the development of transgender people's post-traumatic stress following anti-transgender bias events, I use this section to explore shame, internalized stigma, and their role in post-traumatic stress in greater depth.

\section{Conceptualizing Shame and Shame-Proneness}

Shame and shame proneness have emerged as important constructs in understanding the experience, development, and maintenance of post-traumatic stress. Shame is a self-conscious affect, intense experiences of which cause great psychological distress (Tangney, 1995). It is experienced in response to social threat and is considered an important motivator in both private and interpersonal behavior. Gilbert (1997) proposed distinguishing internal shame and external shame. According to this delineation, external shame relates to the experience of being devalued by others; people 
with high levels of external shame believe that others view them as "inferior, inadequate, disgusting, or weak in some way" (Lee, Scragg, \& Turner, 2001, p. 452). Internal shame relates to a person devaluing themselves, such that individuals with high internal shame view themselves as inferior and view some aspects of themselves as undesirable (Gilbert, 2000). It should be noted that research on trauma-related shame has not supported a distinction between internal and external subtypes (Øktedalen, Hagtvet, Hoffart, Langkaas, \& Smucker, 2014), and studies often evaluate shame as a one-dimensional construct. In their psychological literature-based concept analysis of shame, McFall and Johnson (2009) reported that defining attributes of shame fall into four categories: physical expressions of shame, including blushing, diverting eye contact, and covering one's face or body; feelings of worthlessness, which include feelings of inadequacy, powerlessness, or being bad/wrong; low self-esteem, manifested in insecurity, poor body image, and self-doubt; and alienation, expressed as feeling alone, betrayed, or like an outcast.

Much of the psychological canon around shame has been based on Western and White cultures, but Fessler $(1999,2007)$ argued that shame at its core is a panhuman emotion. Fessler reviewed ethnographic research on shame-like emotions across different cultures and proposed a two-logic conceptualization of shame. Under his conceptualization, shame involves one or both of the following relational experiences, or logics: 1) recognition of one's own inferior social status, and 2) failure to conform to social norms and expectations. Fessler argued that both logics resulted in the same behaviors, which have become identifying features of shame experiences (described above). Although shame is a state individuals experience discretely, shame-proneness is dispositional. Shame-proneness is a construct representing a person's likelihood to 
respond to personal transgressions with shame. The disposition of shame-proneness

indicates that a person is likely to make self-directed attributions about perceived interpersonal failures (Tangney \& Dearing, 2002).

\section{Conceptualizing Internalized Stigma}

Internalized stigma is a belief structure that involves shame experiences. Stigma is routinely defined using Goffman's (1963) conceptualization of a sense that someone has "an attribute that is deeply discrediting" and diminishes a person's value in society (p. 3). Internalized stigma describes a person's belief that they are tainted or less worthy because of an attribute that carries stigma. Ritsher, Otlingam, and Grajales (2003) defined internalized stigma as the "devaluation, shame, secrecy, and withdrawal triggered by applying negative stereotypes to oneself' (p. 3). Internalization of stigma is particularly high risk for people with stigmatized identities that may develop or come into the individual's understanding/focus later in life. Link and Phelan (2001) illustrate this with the example of an individual with mental illness. Prior to developing that mental illness, the person was exposed to the social stigma of mental illness came to adopt negative beliefs about individuals with mental illness into their worldview. When such a person develops mental illness, they have deep-seated negative views about this attribute and about themselves for developing it. Research has documented similar patterns with regard to aging and elderly populations; individuals internalize negative views about older adults while they are young and then age and struggle with damaging, negative age-related beliefs about themselves (Levy, 2009; Levy, Slade, Murpy, \& Gill, 2012). Work on internalized homophobia has theorized that sexual minorities are more affected by antiLGBTQ bias than racial minorities are affected by racist bias because the stigmatized identity of being lesbian, gay, bisexual, or queer is not typically shared with one's family 
of origin. As a result, development of self-worth around an LGBTQ identity and resilience to external stigma are not normal pieces of sexual minorities' development (Szymanski \& Balsam, 2011). Not only are LGBTQ individuals more likely to have internalized negative beliefs before recognizing their identity, they are also less equipped to build resilience to them as they develop said identity (Szymanski \& Balsam, 2011). Additionally, non-normative sexual orientations and gender identities are sometimes believed to be choices made by LGBTQ individuals. Even when an LGBTQ person's internal identity is acknowledged as not being within their control, there is inarguably a degree of control over behavioral decisions, such as engaging in non-heterosexual pairings/sex or expressing a non-normative gender identity. Research has found that for racial minorities, attribution of negative experiences to others' prejudice about their race reduces the emotional impact of discrimination and increases the self-esteem of racial minorities, because they can attribute negative experiences to qualities outside of their control (e.g., Crocker \& Major, 1989; McCoy \& Major, 2003). For many LGBTQ individuals, however, attributing negative experiences to prejudice still places partial responsibility for their suffering on themselves. This may make LGBTQ people more vulnerable to internalization. Suggesting a possible disparity in internalized stigma, strength of transgender identity was not found to be directly related to self-esteem and other positive psychological outcomes (Barr et al., 2016), when the opposite has been found for racial minorities (McCoy \& Major, 2003).

\section{The Role of Shame and Internalized Stigma in the Development of Post-Traumatic}

\section{Stress}

Researchers have found robust evidence of an association between mental health and both experiences of shame and a disposition of shame-proneness. In their meta- 
analysis of 108 studies with 22,411 participants, Sangmoon, Thibodeau, and Jorgensen (2010) found a large effect size between depressive symptoms and external shame and a moderate effect size between depressive symptoms and internal shame. Shame has been found specifically to be associated with PTSD and has emerged as an empirically supported common feature of post-traumatic stress (APA, 2013). In recognition of this empirical support, the diagnostic criteria for PTSD include persistent shame experiences as one of the manifestations of Criterion D (negative alterations in mood and affect) symptoms. Shame is one of the most widely reported emotions in people diagnosed with PTSD (e.g., Resick \& Schnicke, 1992; Reynolds \& Brewin, 1999), and some individuals suffering from PTSD retroactively report experiences of shame rather than fear during the most intense moments of trauma (Grey, Holmes, \& Brewin, 2001; Reynolds \& Brewin, 1999). Shame is also prominently featured in Herman (1992) and Root's (1992) feminist conceptualizations of trauma and its impact, discussed earlier. In Leskela, Deiperink, and Thuras' (2002) study of former prisoners of war determined to be have been exposed to trauma, participants prone to shame-based negative evaluations of the self experienced higher levels of post-traumatic stress, with significant, moderate correlations found between shame-proneness and both avoidance and arousal subscales of the PTSD Checklist-Military for the DSM-IV (PCL; Blanchard, Jones-Alexander, Buckley, \& Forneis, 1996), as well as the overall scale, and a significant, small relationship with the re-experiencing subscale.

Though shame experiences are considered one of the consequences of trauma, many researchers, theorists, and clinicians believe shame to also be a critical component to the development and maintenance of PTSD, which would account for some of the association between PTSD symptoms and shame and shame-proneness. In a longitudinal 
study of trauma survivors, shame experiences within one month of victimization predicted PTSD symptoms at a six-month follow-up (Andrew, Brewin, Rose, \& Kirk, 2000), providing some empirical evidence to this direction of the relationship between shame-proneness and PTSD development. Budden (2009) has posited a socio-emotional model of PTSD, in which shame mediates the relationship between traumas and posttraumatic stress. He argued that many traumatic events, particularly interpersonal traumas, are effectively threats to an individual's social self through either "(1) the experience of acute domination and subjugation; [or] (2) acute violation or erasure of norms, values, and expectations about the world" (p. 1034), and noted the parallel with Fessler's two-logic construction of shame. These attacks on the social self lead to the peritraumatic (occurring during the trauma itself) shame experiences of helplessness and loss of agency and control (in acute domination) and/or exposure, rupture of identity, and loss of moral integrity (in acute violation or erasure of norms and expectations). These theorized effects align with Root's (1992) description of the destruction of dimensions of security. Budden argued that shame becomes an integral part of the traumatic memory. Shame is then experienced with shame-related intrusive cognitions and memories of the event. In the language of Emotional Processing Theory, Budden (2009) proposed that shame is a part of the post-traumatic emotion structure and thus is activated by other aspects of the structure (i.e., triggers; Foa \& Cahill, 2001). Additionally, survivors may experience shame about their post-traumatic shame experiences. As shame is a highly distressing emotional experience (Tangney \& Dearing, 2002), fear of re-experiencing shame leads to hypervigilance, arousal symptoms, dissociative symptoms, and avoidance (Budden, 2009). 
Lee and colleagues (2001) proposed a different etiological model of shame-based PTSD built upon scheme congruence theories of PTSD development. They proposed that following a trauma, a survivor evaluates the meaning of a traumatic event and their response to it using their pre-existing schemas of self, world, and others, and their interrelated perception of the traumatic event. When the individual interprets their trauma experience to convey shame-related meaning (e.g., loss of status, being devalued, sense of self being attacked, loss of social attractiveness), they activate congruent shame schemas and experience pervasive feelings of shame, leading to shame-charged intrusive thoughts and images. The model also offers that schema congruence (the match of meaning derived from the trauma and pre-existing shame schemas) strengthens those shame schemas, which informs later meaning-making should future traumas occur. Thus, this model explains the increased risk of PTSD symptoms with subsequent trauma experiences and in survivors of complex trauma. And importantly, Lee and colleagues (2001) proposed that the strengthening of shame schemas causes the survivor to repeatedly understand their trauma through a shame lens; further attempts to process the trauma then lead to increasingly high levels of shame, which evoke avoidance and concealment behaviors.

This proposed role of shame in mediating avoidance is a key component to both Budden (2009) and Lee and colleagues' (2001) models of post-traumatic stress. As reviewed earlier, avoidance is understood as both a defining feature of post-traumatic stress phenomenology and a developmental factor that facilitates and maintains other post-traumatic stress symptoms. Research has found that intense experiences of shame lead to what Clark and Wells (1995) described as safety behaviors: submission, desire to escape, hiding, and concealment (Gilbert, 2000). These responses to shame are related to 
what theorists understand to be the social function of shame: to protect one's self against social threats. Experiences of shame lead to shame-avoidance behaviors, which involve distancing from the shame-inducing stimuli (Schmader \& Lickel, 2006). Research has established a relationship between shame-proneness and avoidant coping styles, with this relationship partially mediating the association between shame-proneness and psychological distress (e.g., De Rubies \& Hollenstein, 2009; Whiffen \& MacIntosh, 2005).

As internalized stigma involves or leads to shame experiences (Rischer et al., 2003), it is not surprising that research has demonstrated that the relationship between internalized stigma and PTSD symptoms may also be mediated by avoidant coping. In Gold, Dickstein, Marx, and Lexington's (2009) study of lesbian sexual assault survivors, internalized homophobia significantly predicted PTSD symptom severity; experiential avoidance fully mediated this relationship. However, in a similar study of lesbian and gay men, researchers found that internalized homophobia and experiential avoidance differentially mediated the relationship between childhood physical abuse and posttraumatic stress symptoms (Gold, Feinstein, Skidmore, \& Marx, 2011).

\section{Shame Experienced by Transgender People and Its Role in Post-Traumatic Stress}

Internalized transphobia is the internalized stigma a transgender person carries related to their identity and experiences as transgender. It was defined by Hendricks and Testa (2012) as the internalization of negative attitudes about and prejudices against transgender people. Bockting (2015) stated that internalized transphobia is discomfort with one's transgender identity, experience, and history, "as a result of internalizing society's normative gender expectations" (p. 583). In order to measure internalized transphobia, it has been operationalized as shame about one's gender identity or 
expression (Testa et al., 2015). For example, Testa and colleagues' (2015) eight-item scale measuring internalized transphobia includes statements about resentment of one's gender identity or expression, embarrassment, a sense of feeling like a "freak" or outcast, and wishing one's gender was "normal." This language fits with limited qualitative research on transgender people's experiences of internalized transphobia, in which participants discuss feeling or having felt like a "freak" (Budge, Barr, \& Keller, unpublished; FORGE, 2005), as well as defining attributes of shame and internalized stigma (Rischer et al., 2003). Outside of academic literature, internalized transphobia is often discussed as an individual's internal sense of things being wrong with them because they are transgender and/or because they violate gender norms and expectations. Author and transgender woman of color Janet Mock (2014) wrote of her childhood, "[my brother] was held as the standard of acceptable boy behavior; I grew aware of the fact that I negated that standard, and I internalized that on a deep level. I thought that something must be wrong with me..." (p. 31). Ami Kaplan (cited in Schroth, 2015), a transgender psychotherapist who works with transgender clients, wrote the following about internalized transphobia:

Growing up in a culture where this [anti-transgender] attitude is common, you take it in and part of you believes it whether you want to or not. We learn that a certain group of people should be mocked before we know that we are in that group - and then we are stuck in the position of hating something about ourselves. (p. 311) Kaplan's description echoes Link and Phelan's (2001) description of the development of internalized stigma. While internalized transphobia is often discussed in the literature as experienced early in transition with regard to gender appearance and 
incongruence, it can also manifest later. Transgender people who have transitioned and are living as their true gender identity, satisfied with their gender expression, and being perceived as non-transgender, can experience shame about their gender transitions, transgender status, and transgender history (Bockting \& Coleman, 2007). Importantly, this aspect of internalized transphobia has not yet been captured by measures focusing on shame around gender identity or gender expression.

Although the literature is lacking empirical evidence regarding the prevalence and severity of internalized transphobia, papers and books on psychotherapy with transgender clients consistently focus on this construct as a highly common and detrimental experience (e.g., Austin \& Craig, 2015; Bockting, Knudson, \& Goldberg, 2006; Lev, 2004). Developmental models of transgender identity include shame and discomfort with transgender identity as consistent pieces of transgender people's development processes (Bockting \& Coleman, 2007; Lev, 2004; Morgan \& Stevens, 2008; Morgan \& Stevens, 2012).

The relationship between internalized transphobia and mental health has only recently received empirical attention. Beyond Testa and colleagues' (2015) aforementioned research demonstrating an association between proximal stressors and psychological distress, only a handful of studies have examined internalized transphobia. Though few in number, the studies have consistently demonstrated that internalized transphobia is an important construct in understanding transgender people's mental health. Internalized transphobia has been found to be associated with increased risk of suicidality, stress, and psychiatric symptoms (Perez-Brumer, Hatzenbuehler, Oldenburg, \& Bockting, 2015; Sanchez \& Villain, 2009; Testa, Jimenez, \& Rankin, 2014). Qualitative work has found that in the face of trauma and other stressors, transgender 
people employ resilience strategies specifically related to overcoming internalized transphobia and boosting pride and self-worth (Singh, Hays, \& Watson, 2011; Singh \& McKleroy, 2011). Hendricks and Testa's (2012) adaptation of the Minority Stress Model included pride in transgender identity as a resilience factor that protects against negative mental health outcomes, and subsequent research demonstrated support for this relationship (Testa et al., 2015).

\section{Internalized Transphobia and PTSD}

Budden (2009) and Lee and colleagues' (2001) models of shame-mediated PTS can be applied to understand the role of internalized transphobia in anti-transgender biasbased PTS. Anti-transgender bias events act as insidious trauma (Richmond et al., 2012; Root, 1992), which by reminding the transgender person of their devalued status and challenging their self-definition meet Budden's (2009) criteria as attacks on the social self. According to Budden's theory, victims of these bias events would experience identity-specific shame, or internalized transphobia, during the bias event and such shame would become a part of the traumatic memory structure, which would lead to the development of PTS symptoms. Lee's (2001) theory argues that it is not the peritraumatic experience of internalized transphobia that leads to PTS, but subsequent meaning-making about the traumatic event that activates pre-existing internalized transphobia schemas. For example, a transgender person understanding their discrimination as being due to looking like a freak would activate the shame schema of internalized transphobia. Under either model, internalized transphobia would mediate the relationship between bias-based trauma and PTS, but no known empirical work has examined this possible mediation. In the only study examining predictors of internalized transphobia, Testa and colleagues (2015) found correlations between the construct and rejection, victimization, and non- 
affirmation experiences of bias. No studies have examined internalized transphobia and PTS symptoms specifically, though Richmond and colleagues (2012) include internalized transphobia as an important consideration for therapists working with traumatized transgender clients.

\section{Summary}

The literature on transgender mental health indicates that transgender people are at great risk for mental health difficulties, including depression, anxiety, PTSD, and suicidality, and that these risks are at least partially explained by exposure to antitransgender bias (Bockting, Miner, Swineburne Romine, Hamilton, \& Coleman, 2013; dickey et al., 2015; Hendricks \& Testa, 2012; Shipherd et al., 2014; Testa et al., 2015). In their review of the existing literature on anti-transgender bias and transgender people's mental health, Richmond and colleagues (2012) proposed conceptualizing antitransgender bias as potentially traumatizing. Defining bias experiences as trauma requires an expansion of mainstream definitions of post-traumatic stress that are contingent upon exposure to a life-threatening or sexually violent event (so-called Criterion A events, as the trauma events must meet the requirements delineated in Criterion A of the DSM-5 PTSD diagnosis). For decades, camps of trauma psychologists have been calling for such an expansion, and research has demonstrated that non-Criterion A events are uniquely associated with clinically significant elevations in PTS symptoms (e.g., Carlson \& Dalenberg, 2000; Gold et al., 2005; Long et al., 2000; Mol et al.,2005; Van Hooff, 2009). Psychologists tasked with updating the PTSD criteria for the DSM-5, however, rejected requests for Criterion A expansion or elimination (Friedman \& Resik, 2014). This tension is part of a long history of medical, scientific, and psychological debate over determining the validity of trauma sequelae and their sources (Herman, 1997; Weisath, 2014). For 
example, only in the late 1970s after intense advocacy efforts from veterans and women were the relationships between the symptoms of post-traumatic stress and combat and rape accepted by mainstream psychologists (Herman, 1997; Van der Kolk, 2007). As that shift in the defining and understanding of trauma was critical in veterans and sexual assault survivors gaining access to effective treatment and relief, understanding bias events as potentially traumatic would depathologize and destigmatize oppressed individuals who struggle with mental health and allow for the development of more effective mental health care (Richmond et al., 2012). As alluded to in this summary and explored in the literature review, conceptualizing anti-transgender bias as potentially traumatic for transgender people is supported by the literature.

While mainstream fear- and anxiety-based models of trauma and PTSD propose that PTS develops due to fear associated with threats to one's physical self (e.g., Foa \& Cahill, 2001; Foa \& Kozack, 1985; Kilpatric et al., 1985), Richmond and colleagues' (2012) trauma framework for transgender mental health is built upon trauma theory positing that perceived threats to one's social self can elicit similar symptoms. Namely, Root's (1992) construct of insidious trauma - the accumulative traumatic effect of regular bias events - and her feminist reconstruction of post-traumatic stress development offer a model of understanding trauma as the destruction of security dimensions, which can be physical, psychological, or interpersonal. Research establishing relationships between anti-LGBTQ and racist discrimination and PTS symptoms has provided empirical support for the construct of insidious trauma (e.g., Loo et al., 2001; Szymanski \& Balsam, 2011). With regard to transgender populations, one known study has examined the relationship between discrimination and PTSD in a transgender sample; this study found that exposure to discrimination was significantly associated with an increase in PTS symptom 
severity, even after controlling for Criterion A trauma exposure (Reisner et al., 2016).

Reisner and colleagues' study provided transgender-specific evidence for the designation of bias as potentially traumatic.

Richmond and colleagues (2012) also draw upon Herman's (1992) construct of Complex PTSD, a set of symptomatic and characterological changes that can develop following chronic interpersonal traumas (Briere \& Spinazzola, 2005). There are many parallels between oppression-based bias experiences and chronic interpersonal traumas, such as domestic violence (Bryant-Davis \& Ocampo, 2005), suggesting that the posttraumatic stress resulting from bias events may include Complex PTSD symptoms beyond those included in PTSD diagnostic criteria (e.g., depressive symptoms, personality disorder symptoms).

Both mainstream and feminist models of PTSD development recognize that PTS develops in part due to 1) unnecessarily extreme efforts to retain a sense of security following a trauma and 2) avoidance (both conscious and not) that prevents adequate processing of the trauma memories, responses, and/or security behaviors (Foa \& Cahill, 2001; Herman, 1992; Root, 1992). Shame may be a key piece in understanding why some trauma survivors engage in avoidant-based coping (Budden, 2009; Lee et al., 2001). Many trauma survivors report peritraumatic and post-traumatic shame experiences, and shame is associated with increased PTS symptom severity and as well as increased avoidance (e.g., Andrew et al., 2000; Leskela et al., 2002; Resick \& Schnicke, 1992; Reynolds \& Brewin, 1999). Internalized stigma, shame experiences that result from the internalization of negative stereotypes about a held identity, has been found to mediate the relationship between bias event exposure and PTS in LGBTQ samples (Gold et al., 2009; Gold et al., 2011). Theory thus suggests that internalized transphobia, the 
internalized stigma specific to transgender identity, would similarly mediate the relationship between anti-transgender bias event exposure and PTS in a discretely transgender sample. This relationship has not been examined, although internalized transphobia is acknowledged by clinicians as a highly common and difficult struggle for transgender people and is associated with negative mental health outcomes (Austin \& Craig, 2015; Bockting et al., 2006; Lev, 2004; Testa et al., 2015).

\section{Current Study}

Understanding how anti-transgender bias events, and particularly their chronicity and accumulation, are related to post-traumatic stress appropriately destigmatizes and depathologizes transgender people with poor mental health and facilitates better treatment for these individuals (Richmond et al., 2012). This understanding, however, is currently hindered by the dearth of research examining these variables and possible mechanisms of PTS development. In the current study, I examined whether anti-transgender bias exposure is associated with PTS symptom severity and whether this relationship is mediated by internalized transphobia. In my proposed model, consistent with the conceptual frame discussed in this chapter, anti-transgender bias experiences predict PTSD symptom severity after controlling for participants' exposure to potentially traumatic events that they perceive to be unrelated to their transgender identity. Thus, I hypothesized that participants who report higher levels of exposure to anti-transgender bias will display more severe PTSD symptoms, and that this relationship will not be due to increased exposure to bias-unrelated potentially traumatic events. The model is a partial mediation model, with internalized transphobia as the mediator. I hypothesized that increased levels of internalized transphobia will explain some, but not all, of the relationship between anti-transgender bias exposure and PTSD symptoms. These 
hypothesized relationships extended from the theoretical framework - reviewed in this chapter - of the potentially traumatic impact of anti-transgender bias on transgender people, and the role that shame (specifically: internalized transphobia) may play as a mechanism in the development of trauma sequelae related to this bias. I evaluated the accuracy of this model and the strengths of each relationship using structural equation modeling. I explain my methodology, including analytic methods, in greater detail in the following chapter. 


\section{CHAPTER II}

\section{METHODOLOGY}

\section{Data Collection and Sample Demographics}

The study's sample consisted of 575 transgender-identified adults. The number of participants was greater than my goal of $N=500$, which was determined based on (a) Bentler and Chou's (1987) 5 to 1 ratio for sample size to free parameters, which Kenny (2015) recommends to ensure adequate power for measuring SEM model fit and (b) calculations that this number of participants would provide sufficient power to detect parameter effect sizes of 0.2 and greater (Soper, 2016). Of note, sample was not the only consideration regarding the power of my model. As Wolf and colleagues (2015) noted, use of latent variables also increases statistical power by reducing the measurement error in parameter estimates.

Participants were recruited via social networking platforms, including Tumblr, Reddit, Twitter, and Facebook; previous efforts using these sites to recruit transgender participants (Barr, Budge, \& Adelson, 2016) elicited repostings and were highly successful recruitment tools. Additionally, the call for participants was shared via email with LGBTQ and transgender listservs, LGBTQ community and university centers, transgender support and social groups, therapists working with transgender clients, and contacts within the transgender community. These emails also included requests that a participation flier be posted in the centers/offices. Recruitment messages informed potential participants that inclusion criteria were that they had a gender identity that 
differed from the sex they were assigned at birth and were at least 18 years old. Participation was incentivized for participants who wish to share their contact information; this information was taken using a separate survey and was not attached to any participant responses. Participants who wished to share their contact information were entered into a drawing for a $\$ 100$ Amazon gift card.

These recruitment efforts introduce some sampling bias, as the samples recruited via the Internet are inherently convenience samples (Pequegnat et al, 2007) and will be limited to those who have Internet access. Despite these limitations, Miner, Bockting, Romine, and Raman (2013) recommended the use of Internet recruitment when researchers are hoping to gather a broad transgender population, as the transgender (and other stigmatized minority populations) frequently use the Internet to connect with their community and Internet recruitment results in greater sample sizes. By requesting that LGBTQ centers and therapists post recruitment flyers in their offices, I hoped to gain access to transgender people who may not yet be connected with online transgender spaces and/or those without regular Internet access. The use of therapist-based recruitment introduces the potential risk of oversampling the treatment-seeking proportion of the transgender community, however I do not see this as a substantial risk to the validity of this study for two reasons: First, my primary interest in this study is not to capture wholly accurate or representative prevalence/severity levels of the measured variables, but instead to understand their relationships to each other. There is no literature suggesting that treatment-seeking transgender people would have different relationships among bias events, internalized transphobia, and PTS symptoms than non-treatment seeking individuals. Additionally, therapist-based recruitment represented a small portion of recruitment efforts and likely contributed minimally to the sample pool - my email list 
had less than 40 clinicians, and less than five responded indicating that they would make the study known to clients or patients.

Participants were asked to complete a one-time online questionnaire, including a demographic questionnaire and questions about gender-related discrimination, genderrelated rejection, gender-related victimization, non-affirmation, exposure to potentially traumatic events, internalized transphobia, and PTSD symptoms. An initial pool of 729 individuals consented to participation. However, 111 of those did not complete any survey items, and an additional 27 completed only the demographics questions; these cases were removed. I also removed data from 16 participants who indicated that their age was under 18 or who did not disclose their age.

This resulted in a final sample of 575 participants. The majority of the sample reported that they were female assigned at birth $(72 \% ; 27 \%$ reported being male assigned at birth; $1 \%$ reported being assigned intersex at birth). Participants were asked to indicate whether "man," "woman," or "nonbinary" best described their gender identity; $42 \%$ selected nonbinary, $36 \%$ selected man, and $22 \%$ selected woman. All participants also were given the option to report the gender identity labels they used for themselves. Participants reported a wide array of gender identities, including agender, bigender, boi, enby, femme, FTM, genderqueer, gender fluid, gender neutrois, MTF, nonbinary, man, trans feminine, trans man, trans masculine, trans woman, two-spirit, and woman. The mean age of participants was $31.51(S D=11.84)$, with a notably large range: $18-73$. With regard to race and ethnicity, $81 \%$ of the sample were White, Non-Hispanic; $11 \%$ of the sample endorsed multiple racial and ethnic categories; $6 \%$ identified as Latinx/Hispanic; $3 \%$ identified as Black or of African heritage; $4 \%$ identified as having Asian or Pacific 
Islander heritage; $5 \%$ identified as Native American or First Nations; $1 \%$ identified as

Middle Eastern. See Table 3 for full demographic information.

Table 3

Sample Demographics

\begin{tabular}{lrr}
\hline & Frequency & Percent \\
\hline Gender Identity Category & 243 & 42.3 \\
Nonbinary & 206 & 35.8 \\
Man & 126 & 21.9 \\
Woman & & \\
Sex Assigned at Birth & 414 & 72.0 \\
Female & 155 & 27.0 \\
Male & 6 & 1.0 \\
Intersex & & \\
Race and Ethnicity & 467 & 81.2 \\
White, Non-Hispanic & 63 & 11.0 \\
Multiracial/Multiethnic & 32 & 5.6 \\
Latinx/Hispanic & 18 & 3.1 \\
Black & 20 & 3.5 \\
Asian/Pacific Islander & 21 & 4.7 \\
Native/First Islander & 8 & 1.4 \\
Middle Eastern & & \\
Sexuality Category & 278 & 48.3 \\
Queer & 116 & 20.2 \\
Bisexual & 63 & 11.0 \\
Straight & 53 & 9.2 \\
Lesbian & 39 & 6.8 \\
Gay & 22 & 3.8 \\
Asexual & & \\
Highest Education Level & & \\
Currently a student & 17 & 37.7 \\
Did not complete high school & 1.7 \\
Some college & 171 & 29.7 \\
Bachelors degree & 126 & 21.9 \\
Masters degree & 112 & 19.5 \\
Some graduate school & 44 & 7.7 \\
Associates degree & 43 & 7.5 \\
High school diploma/GED & 37 & 6.4 \\
Doctorate & 30 & 5.2 \\
Did not complete high school & 10 & 1.7 \\
Current Employment & & \\
Employed full-time & 274 & 47.7 \\
Employed part-time & 146 & 25.4 \\
Unemployed, not seeking employment & 62 & 16.0 \\
Unemployed, seeking employment & 10.8 \\
Area of Residence & & \\
Urban & 262 & 45.6 \\
& & \\
& &
\end{tabular}




\section{Measures}

In this section, I provide an overview of the measures used, with careful attention to the way they were scored and their psychometric properties, both in pre-existing research and in the current study. I attended to these factors during selection of measures to ensure that I could calculate reliable scores and draw valid conclusions. Note that descriptives (e.g., means and standard deviations) are reported in the Results chapter.

\section{Bias-Related Discrimination, Rejection, and Victimization}

Participants' exposure to and experiences of anti-transgender bias was measured by three subscales of the Gender Minority Stress and Resilience measure (GMSR) developed by Testa, Habarth, Peta, Balsam, and Bockting (2014): gender-related discrimination (five items; sample item: "Because of my gender identity or expression, I have had difficulty finding a bathroom to use when I am out in public"); gender-related rejection (six items; sample item: "I have been rejected or distanced from family because of my gender identity or expression"); and gender-related victimization (five final items; sample item: "I have been threatened with physical harm because of my gender identity or expression"). For each of the subscales, participants responded to items by checking all that apply of the following: Never; Yes, before age 18; Yes, after age 18; Yes, in the past year. Thus, for each item, participants received a score between 0 and 3 , which represented the total number of responses they selected. Testa and colleagues (2014) created items in these scales based on themes identified in a focus group of trans adults (Balsam et al., 2008), which focused on minority stress and experiences frequently identified in national studies of transgender and gender non-conforming people (Beemyn 
\& Rankin, 2011; Grant et al., 2010), as well as adaptations of items from the Sexual Minority Negative Events Scale (Goldblum, Waelde, Skinta, \& Dilley, 2010). With regard to construct validity, Testa and colleagues' (2014) confirmatory factor analysis of the measurement model for the full nine-factor GMSR yielded good model fit, $\chi^{2}$ (1559) $=5922.04, p<.001 ; \mathrm{CFI}=.93 ; \mathrm{RMSEA}=.06$. In their sample of 855 transgender people, internal consistency of the three subscales in question ranged from .61 to .77. These values of Cronbach's alpha are considered lower than typically is desired in scale items. This is to be expected to some degree in measures of bias experiences, as scale developers are attempting to measure a total level of exposure, and exposure to some bias events is not necessarily predictive of exposure to others (Testa et al., 2014). Each subscale produced small but statistically significant correlations with symptoms of depression and anxiety, and both gender-related discrimination and gender-related rejection were statistically significantly correlated with general life stress (Testa et al., 2014). This provides evidence of criterion-related validity.

In the current study, reliability of scores for these subscales was higher than in the prior research, with internal consistency of the subscales ranging from .70 to .81 (genderrelated discrimination: $\alpha=.70$ [95\% CI: .66, .74]; gender-related rejection: $\alpha=.77[95 \%$ CI: .74, .80]; gender-related victimization: $\alpha=.81$ [95\% CI: .78, .83]). In analyses, items within each subscale were summed and entered as an observed indicator to the latent predictor (exogenous) variable of Anti-Transgender Bias Experiences. Subscale values for cases with any missing items on that subscale were considered missing, so as to not introduce measurement error. For details on missingness and how it was addressed in the model, see the Analytic Strategy section of this chapter. 
Non-affirmation of gender identity. The extent to which participants

experienced non-affirmation of their gender identity was measured with the six-item nonaffirmation of gender identity subscale from the Gender Minority Stress and Resilience scale (GMSR; Testa et al., 2014; sample items: "People don’t respect my gender identity because of my appearance or body," "I have difficulty being perceived as my gender accurately"). Participants responded to each item using a 5-point Likert-type scale indicating agreement $(0=$ strongly disagree, $4=$ strongly agree $)$. Providing criterionrelated validity, Testa and colleagues (2014) found statistically significant, medium-sized positive correlations between this subscale and general life stress, depression symptoms, and anxiety symptoms. Items on this subscale demonstrated high internal consistency within that study's transgender sample: $\alpha=.93$. In the current study, items demonstrated similarly high internal consistency ( $\alpha=.92$ [95\% CI: .91, .93]). Item scores were summed and originally entered as an observed indicator to the latent variable of Anti-Transgender Bias Experience, but, as is discussed in detail in the Results chapter, ultimately was entered into the final model as a stand-alone observed predictor (exogenous) variable. Similar to the aforementioned scales, if participants had missing data for any item on this subscale, I treated their overall value for Non-Affirmation as missing.

\section{Anti-transgender bias-unrelated Criterion A PTE exposure. Criterion A}

trauma exposure was measured with the Traumatic Life Events Questionnaire (TLEQ; Kubany et al., 2000). This 22-item scale assesses for the frequency of exposure to a wide range of potentially traumatic events, from natural disasters and motor vehicle accents to being a victim of intimate partner violence and having been hit, beaten up, or badly hurt by a stranger. Participants report the frequency of exposure to each event on a 7-point Likert-type scale $(0=$ never; $6=$ more than five times $)$. This scale is widely used and is 
consistently found to be predictive of PTSD symptoms and diagnoses (Kubany et al., 2000; Peirce, 2009). In their study of transgender people who were male assigned at birth (i.e., transgender women, individuals who cross-dress in women's clothing, and nonbinary/other-identified individuals), Shipherd and colleagues (2011) adapted the survey such that after each item, participants reported whether or not they believed they had experienced the trauma because of their transgender status. This did not seem to disrupt the operation of the scale, as it significantly predicted PTSD symptoms.

Additionally, lending some evidence to the validity of this modification, participants who reported spending more time feminine-presenting than not were more likely to attribute a PTE to anti-transgender bias than participations who reported spending less time feminine-presenting.

In the current study, this measure was used to control for exposure to traumas that are perceived to be unrelated to anti-transgender bias. Thus, for each trauma item, I asked that participants use the 7-point Likert-scale separately for frequency of events due to transgender status and frequency of events unrelated to transgender status. Only the antitransgender bias-unrelated PTE exposure was included in the study's model, although both frequencies were analyzed for the purpose of reporting the prevalence of exposure to trauma. Items from this adapted bias-unrelated TLEQ demonstrated sufficient internal consistency: $\alpha=.78$ [95\% CI: .76, .81]. Item scores were summed to create a scale score, which was entered as an observed predictor (exogenous) variable. Items from the biasrelated TLEQ also had an internal consistency of $\alpha=.78$ [95\% CI: .74, .80].

Internalized transphobia. The internalized stigma or shame that participants feel due to being transgender was measured with the Internalized Transphobia subscale of the GMSR (Testa et al., 2014). This subscale consists of eight items and originally was 
developed by Bockting and colleagues (2014) as the Shame subscale in the Transgender Identity Survey. I adapted scale items to include transition history as a source of shame, when appropriate (sample items: “I often ask myself: Why can’t my gender identity, expression, or history just be normal?;" "I envy people who do not have a transition history or a gender identity/expression like mine"). Participants responded using a 5-point Likert-type scale to indicate their agreement with each statement $(0=$ strongly disagree; $4=$ strongly agree). Without the transition history adaptation, the scale items have demonstrated high internal consistency in transgender populations: $\alpha=.91$ (Testa et al., 2014). Additionally, Testa and colleagues reported a medium to large inverse correlation with gender-related pride, and reported medium to large, positive correlations with general life stress, depression symptoms, and anxiety symptoms, demonstrating good criterion-related validity. In the current study, items (including those adapted to include history) yielded a satisfactory internal consistency: $\alpha=.89$ [95\% CI: .87, .90]. Each item score was entered as an observed indicator to the latent mediator variable of Internalized Transphobia.

PTSD symptoms. Symptoms of post-traumatic stress were measured with the PTSD Checklist for DSM-5 (PCL-5; Weathers, Litz, Keane, Palmieri, Marx, \& Schnurr, 2013), a 20-item scale assessing symptoms that correspond with DSM-5 diagnostic criteria for PTSD. Participants rate how much they were bothered by each symptom within the past month using a 5 -point Likert-type scale $(0=$ Not at all; $4=$ Extremely $)$. Weathers and colleagues adapted the scale from the PTSD Checklist (PCL) developed for DSM-IV to address wording changes and additional symptoms included in the DSM-5 update. Additionally, the earlier edition of the PCL had separate versions for military personnel (PCL-M) and civilians (PCL-C), while the PCL-5 does not. Finally, the 
instructions of the PCL-C and PCL-M asked participants to rank the severity of symptoms with regard to a single traumatic event (the most distressing if they had experienced multiple). The version of the PCL-5 that was used in this study does not instruct participants to focus on a single event.

With regard to validity, the PCL-5 has demonstrated very good convergent and divergent validity. Because the scale was recently developed, there remains debate about the factor structure of the measure. It was designed to have a four-factor structure that aligned with the four symptom clusters in the DSM-5; however, factor analyses have demonstrated that a seven-factor structure is a better fit (Armour et al., 2015; Blevins, Weathers, Davis, Witte, \& Domino, 2015; Liu et al., 2014). Researchers have identified the following seven factors: re-experiencing, avoidance, negative affect, anhedonia, externalizing behaviors, anxious arousal, dysphoric arousal. As is discussed further in the Results chapter, comparative confirmatory factor analyses with data from the current study also indicated that the seven-factor model was the best fit. The PCL-5 has demonstrated high internal consistency when used in non-transgender samples $(\alpha=.94$; Blevins et al., 2015). Although no known study has used the PCL-5 in a transgender sample, Shipherd and colleagues (2011) used the original PCL-C for the DSM-IV, which is highly correlated with the PCL-5 (Blevins et al., 2015), in their study of transgender people. In that study, the scale items demonstrated excellent internal consistency $(\alpha=$ .95 ) and was moderately correlated with Criterion A trauma exposure and strongly correlated with depression measures, though clearly divergent.

Consistent with past research, PCL-5 items demonstrated very high full scale internal consistency in the current study ( $\alpha=.96$ [95\% CI: .95, .97]). I entered each of the seven factors into the model as an observed indicator to the latent outcome (endogenous) 
variable of PTSD Symptom Severity. Values for the observed indicators were calculated by summing item scores, and I handled missing data using the same approach I utilized for the exogenous variables: cases with any item of a factor missing were considered to have missing data for that factor. Again, I did this to reduce the introduction of measurement error.

\section{Analytic Strategy}

I used structural equation modeling (SEM) as the primary analytic technique for this study. SEM allows for the simultaneous modeling of relationships between latent, unobservable variables (e.g., psychological constructs) and their observed indicator variables (e.g., scale item scores), as well as relationships between distinct but related variables, both observed and latent. I selected this technique to assess the relationships between my latent constructs of interest: bias experiences, internalized transphobia, and post-traumatic stress, rather than simply the observed variables (e.g., scale scores), so my model accounted for some of the measurement error inherent in psychological study (Adelson, 2012). All SEM analyses were conducted using Mplus Diagrammer v1.31 (Muthen \& Muthen, 2015). This process is detailed later in this chapter. Demographic, data cleaning, and assumption checking analyses were conducted in SPSS v25.0.0.

\section{Data Cleaning and Assumption Checking}

In their text on statistics and ethics, Vardeman and Morris (2003) argued that "the validity of your results can never be greater than [your] most questionable [assumption]" (p. 26). Two critical pieces of accurate, reliable, and, indeed, ethical data analysis, are assumptions checking and data cleaning, without which researchers risk mis-estimation of outcomes (Osborne, 2013). Prior to conducting analyses, I ensured data were clean and that extreme cases were not exerting undue influence. I designated cases as univariate 
outliers if the absolute value of their z-scores on any observed variable were greater than 3.0. Fourteen such cases were identified and reviewed for possible measurement error; each case was determined to be valid and therefore necessary to retain. To reduce likelihood of undue influence, however, variables with extreme cases were transformed using logarithm (Montgomery, Peck, \& Vining, 2013); these variables were Discrimination, Victimization, and Unrelated TLEQ.

Additionally, I performed assessments to check that data met the following assumptions for Maximum Likelihood estimation (Kline, 2011), the technique I used to estimate my structural equation model: independence of observations and multivariate normality of observed variables. Independence of observations was met via study design: each observation was collected from a unique participant. This was confirmed in review of the data, ensuring there were no observations with duplicate values across the entire demographics section. Additionally, review of sample demographics indicates lack of clustering; for example, analyses of participants' reported zip codes demonstrated that 508 participants reported unique zip codes; the maximum number of participants that shared a zip code was 5 , with only three zip codes reaching this frequency. I assessed each observed variable for normality using calculations of skewness and kurtosis; note that for variables that were transformed, I only evaluated their normality following transformation, as these were the values used in analyses. Data were considered nonnormally distributed if the absolute values of their skewness or kurtosis were greater than 2.0 (Field, 2000 \& 2009; Gravetter \& Wallnau, 2014; Trochim \& Donnelly, 2006). All variables demonstrated sufficient normality by this standard. 
Additional assumptions in Structural Equation Modeling are related to correct specification of the model (Kline, 2011); I addressed this by using theory to guide $a$ priori specification and respecifying only when justified by theory and supporting data.

\section{Missing Data}

Missingness was assessed for every observed variable using the Missing Value Analysis module in SPSS (version 25.0). Percent missing ranged from $0.7 \%$ to $10.3 \%$. Little's test was statistically significant, $\chi^{2}(1084)=1248.06, p<.001$, meaning that data cannot be assumed to be missing completely at random (MCAR). The final scale of the questionnaire, the PCL-5, demonstrated the greatest proportion of missing values $(9.7 \%$ 10.3\%). Because the Mplus default method for handling missing data is FIML, which assumes data are at least missing at random (MAR), I needed to determine as best as I could that data were not missing not at random (MNAR). To do this, I hypothesized that data would be MNAR if participants dropped out of the survey because their PTSD was triggered before they could complete the PCL-5, measuring their PTSD symptoms. This would constitute MNAR data because it would mean that the reason for missing data would have been captured by the missing values. The best way to assess for this scenario was to evaluate whether those who did not complete the PCL-5 had higher TLEQ scores, as previous studies have demonstrated high correlations between the TLEQ and earlier iterations of the $\mathrm{PCL}^{4}$ (e.g., Frazier et al., 2009). Therefore, I created a dummy code for missingness on any PCL-5 items and used this variable to sort participants into groups based on whether or not they had completed all PCL-5 items. I compared these groups'

\footnotetext{
${ }^{4}$ No known research has reported relationships between the TLEQ and version of the PCL-5 that I used in the current study. As discussed earlier, the previous version of the PCL, developed based on DSM-IV criteria is very similar to the PCL-5, and largely measures the same construct of post-traumatic stress, with a small number of items changed to reflect nuanced shifts in diagnostic criteria.
} 
means on each of the other observed variables, and none of the differences were statistically significant; most notably, individuals who failed to complete the PCL-5 did not have statistically significantly higher TLEQ scores than those who did complete the PCL-5, $t(45.36)=-1.54, p=.25$, equal variances not assumed due to significant Levine's test, $F=6.92, p=.01$. Data were thus assumed to be MAR.

\section{Model Specification}

As introduced earlier, I used SEM to model structural relationships between variables of interest, as well as measurement relationships between latent variables and their observed indicators. In order to test this model, I followed the two-step procedure recommended by Anderson and Gerbing (1988), first establishing a measurement model that demonstrated adequate fit, before incorporating path analysis techniques to build and estimate structural paths. To determine how well the models "fit to" or represented the data, I reviewed three primary fit indices, as recommended by Kline (2010): the TuckerLewis index (TLI), the comparative fit index (CFI), the root mean square error of approximation (RMSEA). The TLI and CFI are incremental indices which indicate how well the model fits the data compared to a null model. For these indices, which range from 0 to 1 , I followed field norms and considered values greater than .90 to indicate adequate fit and values greater than .95 to indicate good fit ( $\mathrm{Hu} \&$ Bentler, 1999; as cited in Adelson, 2012). The RMSEA is a parsimony-adjusted index that estimates the amount of error while accounting for degrees of freedom and sample size. An RMSEA value of 0 indicates perfect fit. Again following field norms, I considered values less than .08 to indicate adequate fit, with values less than .05 as the ideal indicator of good fit (MacCallum, Browne, \& Sugawara, 1996, as cited in Adelson, 2012). Additionally, I reported $\chi^{2}$ fit index values. This statistic tests the significance of difference between the 
covariance-variance matrix implied by the model and that actually found in the data, with an insignificant value indicating good fit. This is not often a good measure of SEM model fit, because it is affected by multivariate nonnormality and large numbers of parameters (Kline, 2010), and thus I did not consider significant $\chi^{2}$ values to necessarily indicate poor fit. Finally, to compare the fit of nonnested models, I also reviewed the Akaike information criterion (AIC) and the Bayesian information criterion (BIC), which are measures of relative goodness of fit, with smaller comparative values of each indicating better fit (Kline, 2010). Burham and Anderson (2002) demonstrated that AIC differences that are greater than 10 indicate that models are meaningfully different, and Raftery (1995) suggest that BIC differences greater than 10 provide very strong evidence favoring the model with smaller BIC; these were the standards by which I determined comparative fit.

Specifying the measurement model involved multiple steps. Before I could build the full measurement model, I used SEM to compare the fit of confirmatory factor analyses (CFAs) of the PCL-5 to determine the measure's factor structure. This was necessary because the instrument itself is relatively new and its factor structure is still under debate (Blevins et al., 2015). Analyses from Blevins and colleagues' (2015) evaluation of factor structure were replicated, such that 4-factor, 6-factor, and 7-factor models were compared (see Figures 1-3). The 4-factor model included factors representing the four symptoms clusters in the DSM-5 diagnostic criteria: Reexperiencing (five items), Avoidance (two items), Negative Alterations in Mood and Cognition (seven items), and Hyperarousal (six). In the 6-factor model, three items were moved from Negative Alterations in Mood and Cognition to a new factor: Anhedonia, and the Hyperarousal factor was divided into Dysphoric Arousal (four items) and 
Anxious Arousal (two items). In the 7-factor model, two items from Dysphoric Arousal were moved to a new factor: Externalizing Behaviors. I compared the fit of these models using the fit statistics detailed above. As is discussed in the Results chapter, the 7-factor model was favored.

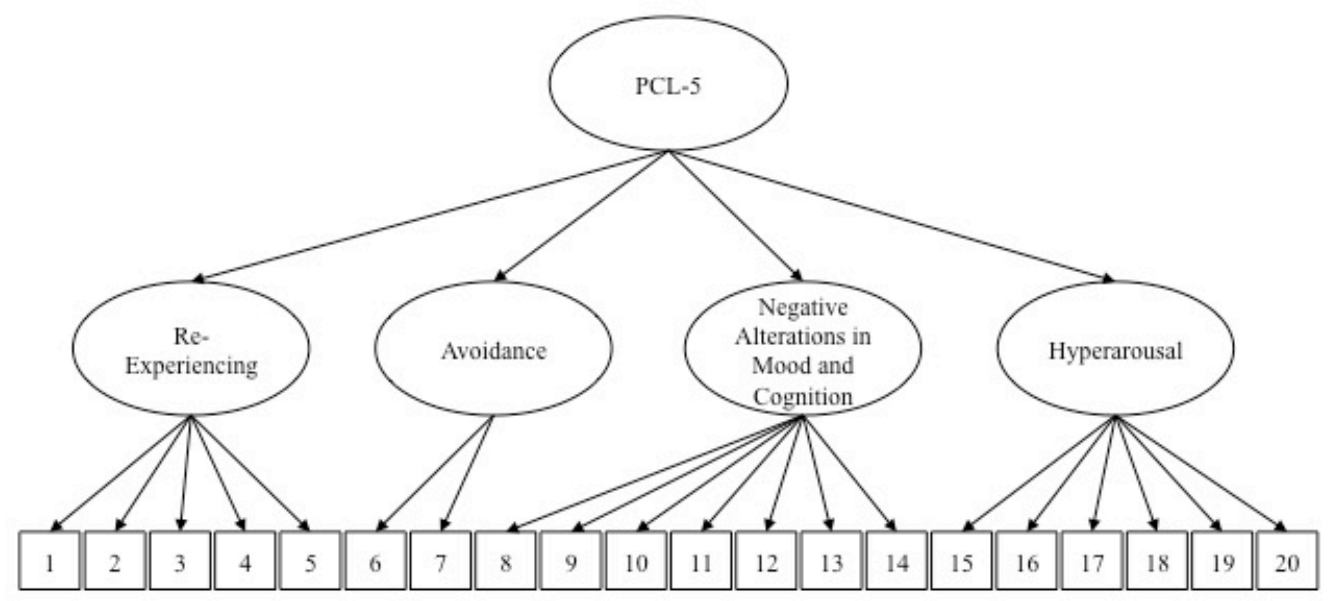

Figure 1. 4-Factor PCL-5 Model

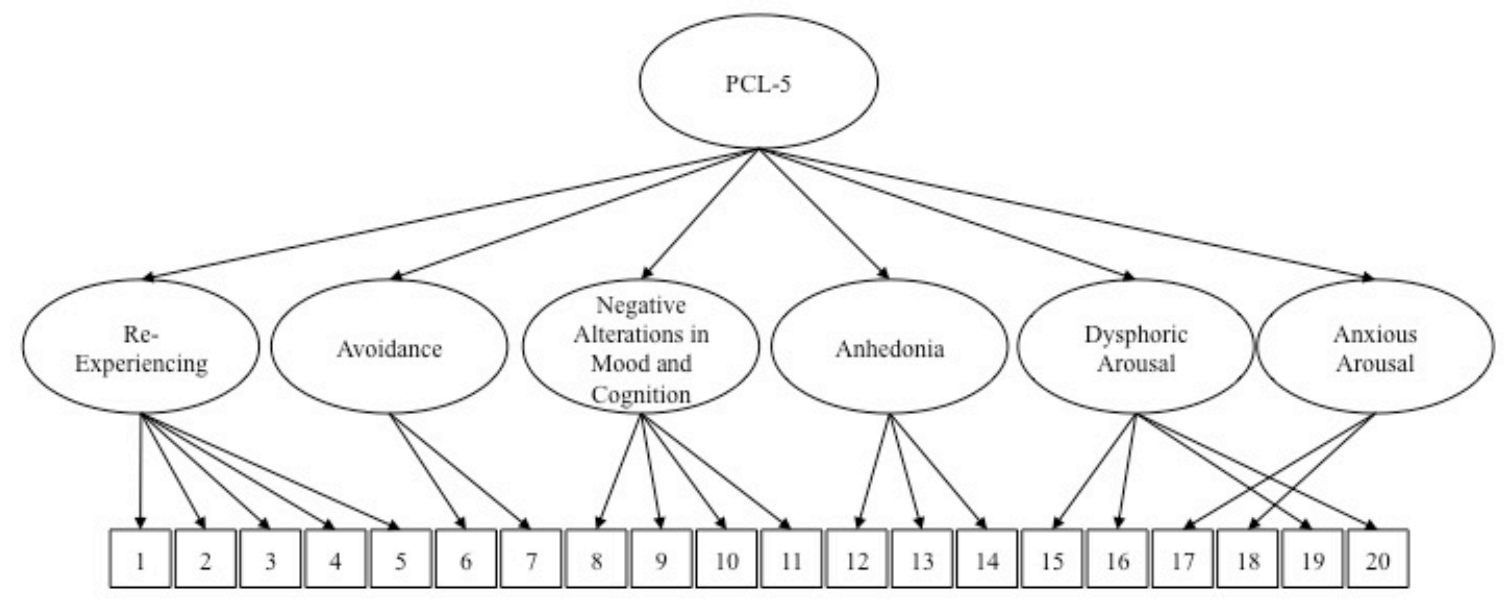

Figure 2. 6-Factor PCL-5 Model 


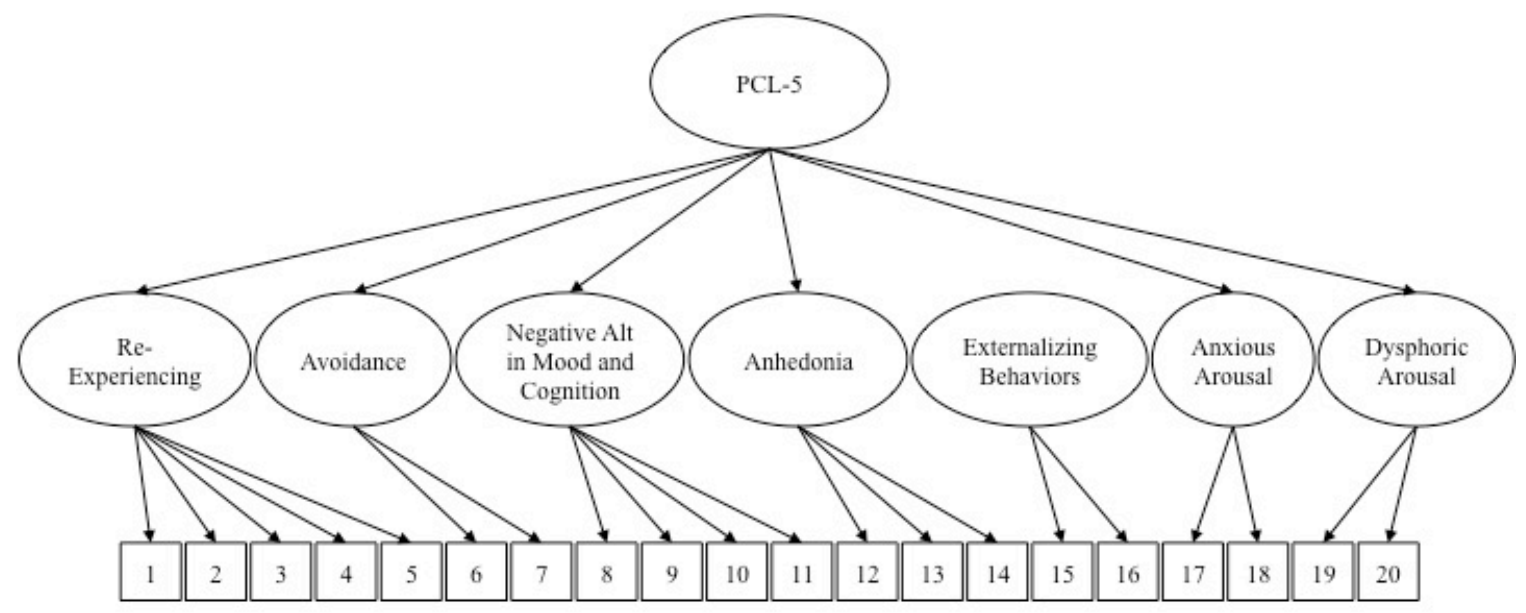

Figure 3. 7-Factor PCL-5 Model

Once the factor structure of the PCL-5 was determined, I tested the a priori measurement model. The a priori measurement model (Measurement Model 1; see Figure 4) was based on the scales' validated factor structures, including the 7-factor structure of the PCL-5. Potentially traumatic events perceived as unrelated to transgender identity, expression, or history were controlled for by including the bias-unrelated subscore of the TLEQ as an observed variable (Unrelated TLEQ). Anti-Transgender Bias Experiences was a latent variable with the four scales of non-affirmation and transgender-related victimization, rejection, and discrimination as its observed indicators. Internalized transphobia was a latent variable with each of the eight items of the internalized transphobia scale as its observed indicators. Post-traumatic stress (PTSD Symptom Severity) was a latent variable with each of its seven factors included as observed indicators. This model included correlations between Bias Experiences, Internalized Transphobia, PTSD Severity, and Unrelated TLEQ, but no structural paths were included, as this was purely a measurement model. 


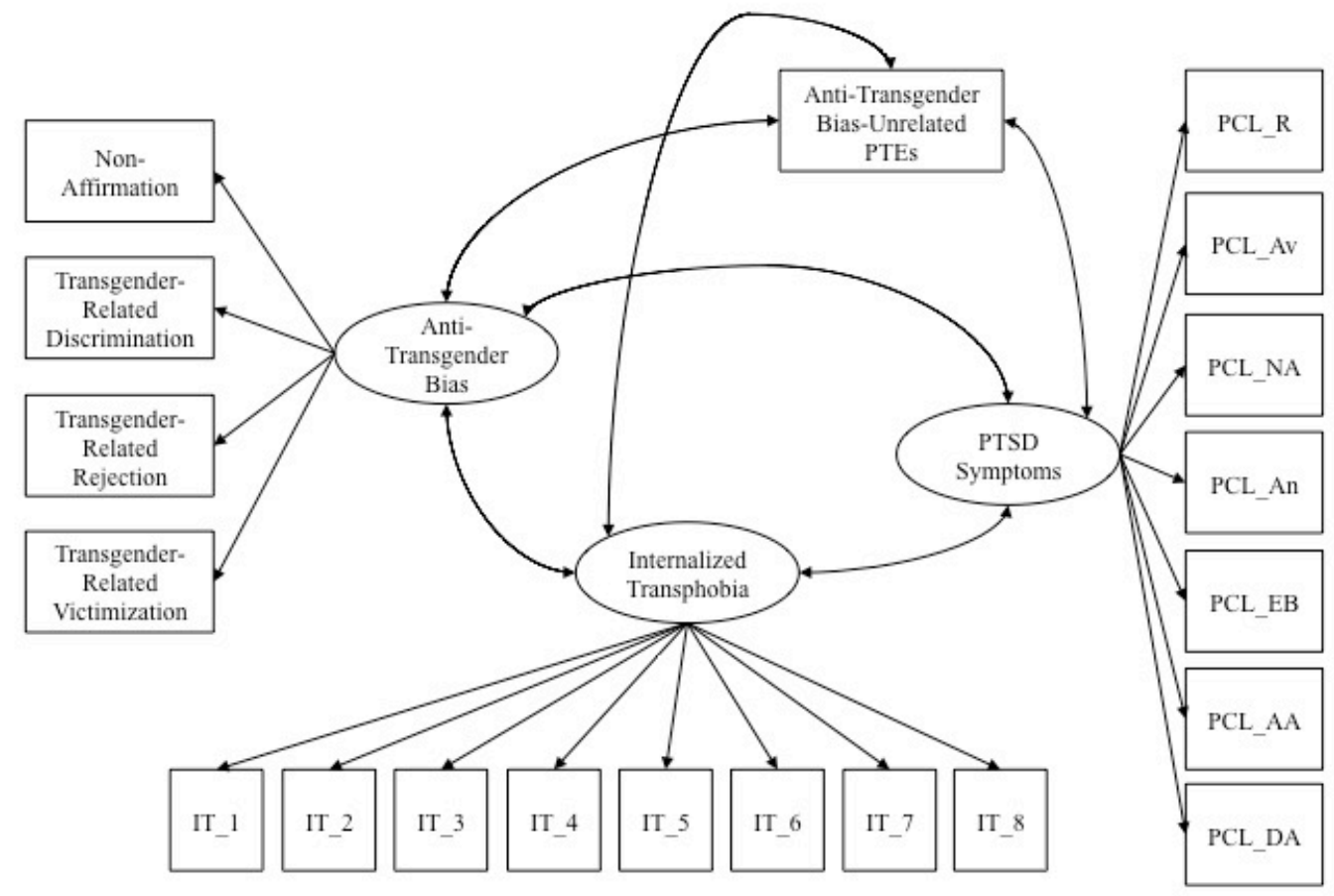

Figure 4. A Priori Measurement Model

As is detailed in the Results chapter, I respecified this model based on evaluation of factor loadings and modification indices, when theoretically appropriate. Multiple researchers have noted that SEM is an approach for testing how well a model fits the data and should not be used to build a model to fit data - this can over-specify a model to the specific sample and damages validity (e.g., Adelson, 2012; Kline, 2010). I followed recommendations to only make model respecifications that can be justified theoretically.

After respecifying the measurement model and determining it had adequate fit, I incorporated structural paths using Bollen's (1988) method of first testing a saturated model with all possible structural paths to ensure that significant paths were not excluded from estimation. I then reviewed each of my hypothesized paths for statistical significance, to ensure that the final hybrid model only included parameters of interest. Although each of my hypothesized paths were statistically significant (see Results for 
details), had they not been, I likely would have been motivated by my research questions to retain them in a final model. I evaluated this final hybrid model for fit according to the previously discussed thresholds and standards.

Finally, I estimated the size and significance of all parameters of the final model using Maximum Likelihood, because all of my dependent variables were continuous and I met the assumption of MAR, as detailed earlier.

\section{Evaluating Indirect Relationships.}

In addition to the direct paths I estimated, my model included two indirect relationships: between Non-Affirmation and PTSD Symptom Severity and between Bias Experiences and PTSD Symptom Severity, both of which were hypothesized to be partially mediated by Internalized Transphobia. Preacher and Hayes (2008) recommend multiple techniques that researchers can use in order to evaluate the significance of indirect relationships while taking into account the nonnormal distribution of the indirect effect, one of which is bootstrapping. Bootstrapping involves repeatedly drawing samples from the full data set and estimating the indirect effect in each resampled data set. I used the MODEL INDIRECT and BOOTSTRAP functions in Mplus and was able to evaluate the size and significance of both the indirect relationships (via bootstrapping) and the total relationships (via normal-theory methods). 


\section{CHAPTER III}

\section{RESULTS}

\section{Descriptives and Frequencies}

It is worth highlighting some of the descriptive and frequency statistics in participants' scores on the measures used in this study (See Appendix A for correlations, means, and standard deviations for each variable). The rates of exposure to antitransgender bias were incredibly high, with $92.6 \%$ reporting at least one experience of transgender-related discrimination, 94.2\% reporting at least one experience of antitransgender rejection, and $78.9 \%$ reporting at least one experience of anti-transgender victimization. Note that these measures produced values on continuous scales, such that these high rates of exposure to at least one type of bias did not result in ceiling effects.

Participants' total scores on the PCL-5 indicated that a large portion of the sample struggled with post-traumatic stress $($ Median $=27.00 ; M=29.99, S D=20.77)$. The National Center for PTSD (2017) recommends a preliminary cutoff of 33 for the overall PCL-5 score, with scores of 33 or greater representing clinically significant overall PTSD symptom severity. Note that possible range is 0 to 80 . Nearly half (43\%) of participants in this study had scores of 33 or greater. Because the PCL-5 was designed to evaluate the symptoms listed as diagnostic criteria for PTSD in the DSM-5, participant responses can also be used to make provisional diagnoses (National Center for PTSD, 2017). Per the recommended guidelines, I treated each item with a symptom severity rating of "Moderately" or higher as an endorsed symptom and identified participants who 
endorsed symptoms across criterion clusters (at least one each in criteria B and C, and at least two each in criteria $\mathrm{D}$ and $\mathrm{E}$ ). By this approach, $44.2 \%$ of participants met criteria for a provisional diagnosis of PTSD, with $62.8 \%$ endorsing at least one symptom of Criterion B, 59.9\% endorsing at least one in Criterion C, $66.1 \%$ endorsing at least two in Criterion D, and $60.6 \%$ endorsing at least two in Criterion E.

Rates of exposure to potentially traumatic events (PTE) were also high, with the vast majority of participants $(93.1 \%)$ reporting exposure to a PTE related to being transgender and $93 \%$ reporting exposure to a PTE unrelated to being transgender. See Table 4 for frequencies for each PTE.

Table 4

Trauma Exposure

\begin{tabular}{|c|c|c|c|c|}
\hline \multirow[t]{2}{*}{ Event } & \multicolumn{2}{|c|}{$\begin{array}{c}\text { Exposure } \\
\text { Related to Bias }\end{array}$} & \multicolumn{2}{|c|}{$\begin{array}{c}\text { Exposure } \\
\text { Unrelated to Bias }\end{array}$} \\
\hline & $\underline{n}$ & $\underline{\%}$ & $\underline{n}$ & $\underline{\%}$ \\
\hline Natural Disaster & 9 & 1.67 & 80 & 32.78 \\
\hline MVA & 4 & 0.74 & 97 & 18.03 \\
\hline Accident & 7 & 1.30 & 46 & 8.56 \\
\hline Combat trauma & 4 & 0.70 & 30 & 5.61 \\
\hline Unexpected sudden death of loved one & 23 & 4.28 & 266 & 49.44 \\
\hline Robbed or witnessed robbery with weapon & 12 & 2.23 & 40 & 7.43 \\
\hline Assault by stranger & 80 & 14.84 & 49 & 9.11 \\
\hline Witness assault by stranger & 15 & 2.81 & 66 & 12.38 \\
\hline Threatened serious physical harm/death & 156 & 29.27 & 136 & 25.52 \\
\hline Physical abuse in childhood & 64 & 12.05 & 126 & 23.73 \\
\hline Witnessed family violence in childhood & 18 & 3.39 & 72 & 13.56 \\
\hline Domestic Violence & 41 & 7.71 & 85 & 15.98 \\
\hline Sexual abuse before age 13 (by older perp) & 32 & 9.12 & 96 & 18.08 \\
\hline Sexual abuse before age 13 (by other child) & 24 & 4.51 & 72 & 13.53 \\
\hline Sexual abuse between age 13 and age 18 & 58 & 10.94 & 108 & 20.38 \\
\hline Sexual abuse/assault after age 18 & 86 & 16.17 & 108 & 20.30 \\
\hline Stalked & 71 & 13.32 & 114 & 21.39 \\
\hline Life threatening illness & 16 & 3.00 & 268 & 50.28 \\
\hline Miscarriage & 5 & 0.94 & 22 & 4.14 \\
\hline Abortion & 5 & 0.95 & 11 & 2.09 \\
\hline Other & 30 & 5.64 & 131 & 24.62 \\
\hline
\end{tabular}




\section{Building the Measurement Model}

I first compared CFAs of the 4-factor, 6-factor, and 7-factor models of the PCL-5. Without respecificaiton (i.e., without allowing for correlated errors), all three models demonstrated sufficient fit (see Table 5). The 7-factor model had the smallest AIC and BIC scores, the smallest RMSEA, and the greatest CFI and TLI, and thus was favored. ${ }^{5}$ This is consistent with Blevins et al.'s (2015) findings. The 7-factor model was included in all subsequent analyses.

Table 5

Model Fit Indices for Competing Factor Structures of the PCL-5

\begin{tabular}{lccccccc}
\hline Model & $\boldsymbol{\chi}^{2}$ & df & CFI & TLI & RMSEA & AIC & BIC \\
\hline 4-Factor & $860.17^{*}$ & 166 & .91 & .90 & $.09(.084, .096)$ & 28738.31 & 29010.43 \\
6-Factor & $626.74^{*}$ & 164 & .94 & .93 & $.07(.068, .080)$ & 28508.89 & 28789.52 \\
7-Factor & $602.17^{*}$ & 163 & .95 & .94 & $.07(.066, .078)$ & 28486.32 & 28771.19 \\
\hline
\end{tabular}

I then tested the a priori measurement model (Measurement Model 1). This initial model did not demonstrate adequate fit; its fit indices fell just below or above cutoffs for good fit (see Table 6). The items on the Internalized Transphobia scale performed as expected, with all of them loading onto the latent variable of Internalized Transphobia ( $\beta$ range: $0.62-0.85)$. PCL-5 factors also performed as expected with $\beta$ values of $0.70-0.91$. The anti-transgender bias factors did not perform as expected, however. Discrimination, rejection, and victimization all loaded onto the latent variable of Bias Experiences $(\beta=$ $0.69, \beta=0.83$, and $\beta=0.74$, respectively), but Non-Affirmation failed to adequately load $(\beta=0.19)$.

${ }^{5}$ Comparisons also were made between models that had been respecified to include correlated errors that were indicated by a priori theoretical justification and modification indices with values greater than 10.0. Among the respecified models, the 7-factor model was still favored. 
To address this, I respecified Measurement Model 2 to include Non-Affirmation as a distinct observed exogenous variable and removed the parameter that had made it an indicator of Bias Experiences in Measurement Model 1. There is little literature on nonaffirmation and no known work on the ways it is similar to or different from antitransgender bias. However, my decision can be justified by examination of scale items, which indicate that the other indicator variables for Bias Experiences are measured with items that involve attribution judgments (X happened because of my gender identity, expression), while Non-Affirmation items do not. I further elaborate on the theoretical difference between these constructs in the Discussion chapter. I considered removing Non-Affirmation in its entirety but decided that its relationships to Internalized Transphobia and Post-Traumatic Stress were both interesting and relevant to my study. This decision was based both on theory and on my data: First, lack of affirmation is a distressing relational experience that can be explained using the feminist and multicultural models of oppression-based trauma (Bryant-Davis \& Ocampo, 2006; Richmond et al., 2012; Root, 1992). Second, my sample had a mean non-affirmation score of $13.38(S D=7.53)$ out of a range of $0-24$, indicating that non-affirmation experiences may be typical for transgender folks - which is certainly consistent with transgender narratives (e.g., Mock, 2016). Removing the parameter between NonAffirmation and Bias Experiences improved model fit, such that RMSEA moved into adequate ranges, but other indices fell short of adequate fit levels $(\mathrm{CFI}=.89$; TLI $=.88$; see Table 6).

I next reviewed the modification indices. Before evaluating Measurement Model 1 and 2, I had proposed a number of correlated errors that were theoretically justified. I examined the output to determine which of the correlated errors I had hypothesized as 
possibly needing exceeded 10.0. I incorporated eight correlated errors into the model, adding correlations stepwise (prioritizing by size of modification index) until no a priori modification index was greater than 10.0. Measurement Model 3 includes those additional correlated errors. Fit indices suggested this model had good fit, and review of the changes to AIC and BIC suggested that the improved fit was worth the increased number of evaluated parameters (see Table 6).

Table 6

Model Fit Indices for Measurement Models

\begin{tabular}{lccccccc}
\hline Model & $\boldsymbol{\chi}^{\mathbf{2}}$ & df & CFI & TLI & RMSEA & AIC & BIC \\
\hline Model 1 & $736.39^{*}$ & 165 & .89 & .88 & $.08(.072, .084)$ & 38227.73 & 38510.42 \\
Model 2 & $671.40^{*}$ & 162 & .90 & .89 & $.07(.068, .080)$ & 38163.11 & 38458.85 \\
Model 3 & $439.43^{*}$ & 154 & .95 & .93 & $.06(.051, .063)$ & 37921.01 & 38251.54 \\
\hline
\end{tabular}

${ }^{*} p<.001$

Note. Model 1: CFAs based on measures' proposed factor structures; Model 2: Model 1 with Non-Affirmation removed as indicator for latent construct of Anti-Transgender Bias Experiences and enter it as an observed exogenous variable; Model 3: Model 2 with correlated errors included.

\section{Building the Hybrid Model}

As the second step in Anderson and Gerbing's (1988) methodology, I incorporated structural parameters into the final measurement model. Per Bollen's (1989) method for specifying structural parameters, I first tested a saturated model to ensure that significant paths were not excluded from estimation. The only non-hypothesized path was from unrelated PTEs to Internalized Transphobia. This path was not statistically significant $(p=.79)$, as hypothesized, and was not included in subsequent analyses.

In my next analysis, I determined the fit of the overall model and tested hypothesized structural parameters for significance. In this model, the latent variable of Bias Experiences was hypothesized to predict the latent constructs of Internalized Transphobia and PTSD Severity, as was the observed variable of Non-Affirmation. 
Internalized Transphobia was also hypothesized to predict PTSD Severity, and thus it partially mediated the relationship between Non-Affirmation and PTSD Severity and the relationship between Bias Experiences and PTSD Severity. Bias-Unrelated TLEQ Scores was a control variable hypothesized to predict PTSD Severity. Each of the proposed pathways was statistically significant $(p<.05)$, and the fit indices indicated adequate to good overall model fit $\left(\chi^{2}(155)=439.65, p<.001 ; \mathrm{CFI}=.95 ; \mathrm{TLI}=.93 ; \mathrm{RMSEA}=.06\right.$, CI90 [.050, .063]. Therefore, this was my final model (see Figure 5) used to estimate direct and indirect parameters.

\section{Model Estimation}

The final model was run with a bootstrap sample draw of 1000. It demonstrated good fit: $\left(\chi^{2}(155)=471.21, p<.001 ; \mathrm{CFI}=.95 ; \mathrm{TLI}=.94 ; \mathrm{RMSEA}=.06, \mathrm{CI} 90[.054\right.$, .066]. All hypothesized relationships were statistically significant. See Table 7 for a summary of parameter estimates. Both Bias Experiences and Non-Affirmation had statistically significant direct relationships with Internalized Transphobia $(\beta=0.18, p<$ .01 , and $\beta=0.22, p<.001$, respectively) and Post-Traumatic Stress Symptoms $(\beta=0.17$, $p<.001$ and $\beta=0.26, p<.001$, respectively). Internalized Transphobia also predicted PTSD Symptoms to a statistically significant degree $(\beta=0.21, p<.001)$. 


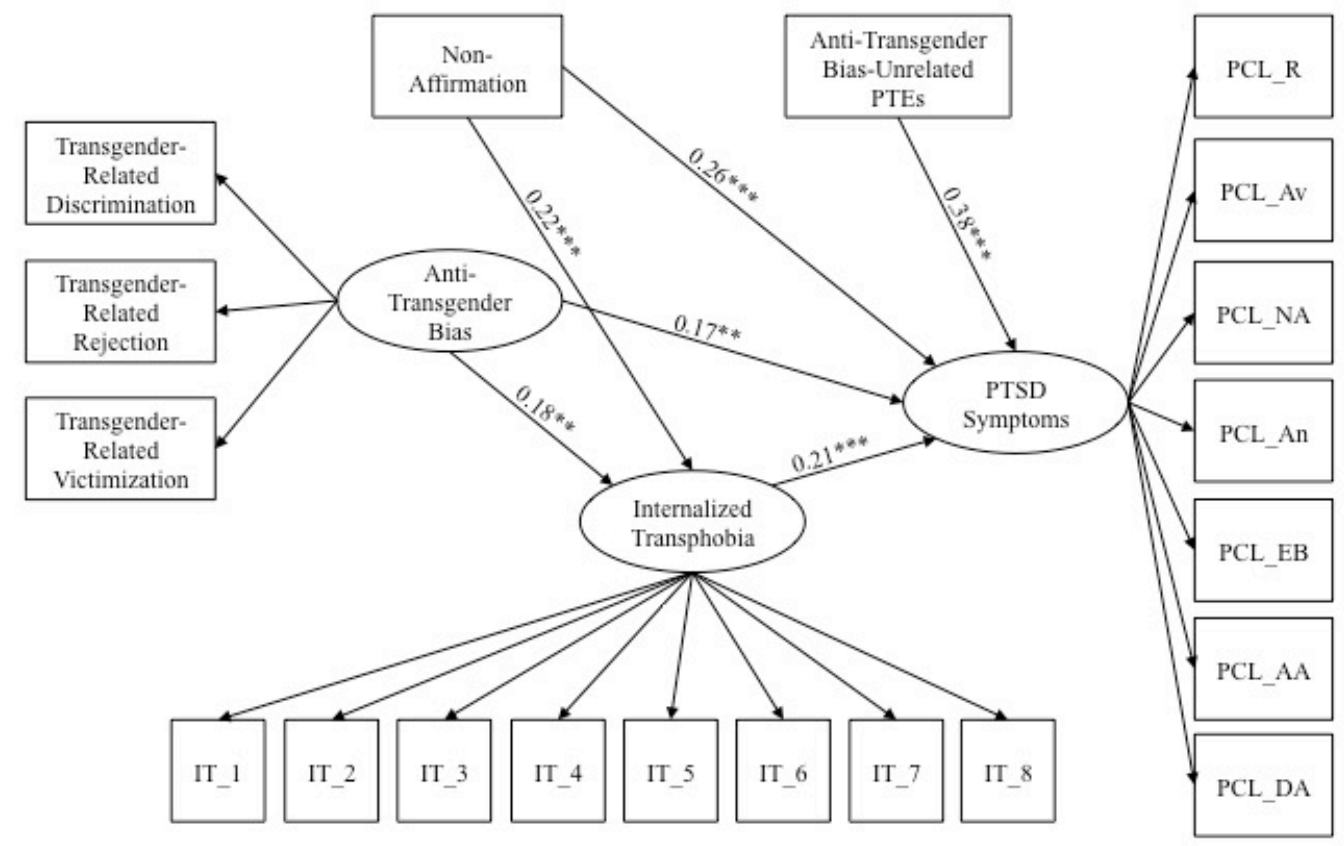

Figure 5. Final Hybrid Model with Standardized Path Estimates

Indirect relationships were also statistically significant (see Table 7 for summary). The indirect relationship between Bias Experiences and PTSD Symptoms was statistically significant $(\beta=0.04, p<.01)$. Similarly, there was a statistically significant indirect relationship between Non-Affirmation and PTSD Symptoms $(\beta=0.05, p<.01)$. Thus, the total relationship between Bias Experiences and PTSD Symptoms is small, statistically significant $(\beta=0.21, p<.001)$, and partially mediated by Internalized Transphobia (proportion of total effect mediated $=15.24 \%$ ). The total relationship between Non-Affirmation Experiences and PTSD Symptoms is also statistically significant but moderate in size $(\beta=0.30, p<.001)$ and also partially mediated by Internalized Transphobia (proportion of total effect mediated $=15.13 \%$ ). 
As expected, the control variable of potentially traumatic events unrelated to transgender bias (Unrelated TLEQ) had a moderate relationship with PTSD Symptoms ( $\beta$ $=0.38, p<.001)$. Of note, the correlation between Unrelated TLEQ scores and Bias Experiences was statistically significant and moderate in size $(\beta=0.37, p<.001)$. The correlation between Unrelated TLEQ and Non-Affirmation was not significant $(\beta=0.06$, $p=.11)$. There was a small but statistically significant correlation between Bias Experiences and Non-Affirmation experiences $(\beta=0.17, p<.001)$.

Table 7

Model Results

\begin{tabular}{|c|c|c|c|}
\hline \multicolumn{4}{|c|}{ Direct Path Coefficients } \\
\hline$\underline{\mathrm{DV}}$ & IV & $\underline{\beta}$ & $\underline{S E}$ \\
\hline \multirow[t]{2}{*}{ Internalized Transphobia } & Non-Affirmation*** & 0.22 & $\overline{0.04}$ \\
\hline & Bias Experience** & 0.18 & 0.05 \\
\hline \multirow[t]{4}{*}{ PTSD Symptoms } & Non-Affirmation $* * *$ & 0.26 & 0.04 \\
\hline & Bias Experience** & 0.17 & 0.05 \\
\hline & Internalized Transphobia*** & 0.21 & 0.04 \\
\hline & Unrelated TLEQ $* * *$ & 0.38 & 0.04 \\
\hline \multicolumn{4}{|c|}{ Indirect Path Coefficients } \\
\hline$\underline{\mathrm{DV}}$ & $\underline{\mathrm{IV}}$ & $\underline{\beta}$ & $\underline{S E}$ \\
\hline \multirow[t]{2}{*}{ PTSD Symptoms } & Non-Affirmation $\overline{* * *}$ & 0.05 & $\overline{0.01}$ \\
\hline & Bias Experience** & 0.04 & 0.02 \\
\hline \multicolumn{4}{|c|}{ Factor Loadings } \\
\hline Latent Variable & Observed Variable & $\underline{\beta}$ & $\underline{S E}$ \\
\hline \multirow{3}{*}{$\overline{\text { Bias Experience }}$} & Discrimination & 0.69 & 0.03 \\
\hline & Rejection & 0.83 & 0.03 \\
\hline & Victimization & 0.75 & 0.03 \\
\hline \multirow{8}{*}{ Internalized Transphobia } & IT_1 & 0.69 & 0.03 \\
\hline & IT_2 & 0.79 & 0.02 \\
\hline & IT_3 & 0.81 & 0.02 \\
\hline & $\mathrm{IT}^{-} 4$ & 0.75 & 0.02 \\
\hline & IT_5 & 0.65 & 0.03 \\
\hline & IT_6 & 0.63 & 0.03 \\
\hline & $\mathrm{IT}_{-}^{-} 7$ & 0.61 & 0.03 \\
\hline & IT_8 & 0.61 & 0.03 \\
\hline \multirow[t]{4}{*}{ PTSD Symptoms } & PCL-5: Re-experiencing & 0.88 & 0.01 \\
\hline & PCL-5: Avoidance & 0.81 & 0.02 \\
\hline & PCL-5: Negative Affect & 0.90 & 0.01 \\
\hline & PCL-5: Anehedonia & 0.76 & 0.02 \\
\hline
\end{tabular}


PCL-5: Externalizing Behaviors

PCL-5: Anxious Arousal

PCL-5: Dysphoric Arousal
0.69

0.78

0.77

0.03

0.02

0.02

$* * p<.01 . * * * p<.001$

$R^{2}$ values for the latent endogenous variables were statistically significant. The model explained $41.5 \%$ of the variance in PTSD Symptom Severity $(p<.001)$ and 9.5\% of the variance in Internalized Transphobia $(p<.01)$. 


\section{CHAPTER IV}

\section{DISCUSSION}

The purpose of this study was to evaluate my hypotheses that (1) levels of exposure to anti-transgender bias would predict PTSD symptom severity in transgender adults even after controlling for trauma exposure that was not related to anti-transgender bias, and (2) this relationship would be partially mediated by participants' internalized transphobia. Data analyses supported these hypotheses and yielded a number of important findings, which I discuss in this section in the context of the theoretical frameworks presented in Chapter 1, and with a focus on implications for theory, research, and clinical work.

\section{Difference Between Anti-Transgender Bias and Non-Affirmation}

Prior to discussing the main findings of this research, it is important to first theoretically differentiate between non-affirmation and anti-transgender bias experiences, as measured by transgender-related victimization, rejection, and discrimination. In the $a$

priori model, based on the Gender Minority Stress and Resilience Scale (GMSR; Testa et al., 2015), non-affirmation was an indicator of the latent variable of anti-transgender bias experiences. However, in the current study, analyses during model respecification demonstrated that non-affirmation was not an adequate factor in this construct, based on fit indices and poor factor loadings. In lieu of this structure, final analyses in this study included non-affirmation as a distinct observed exogenous variable. Thus my research 
questions shifted to consider the relationships that both anti-transgender bias experiences and non-affirmation had with internalized transphobia and post-traumatic stress.

It ultimately is conceptually helpful to consider non-affirmation as distinct from bias experiences, and not altogether surprising that analyses differentiated between these measures. The items on the three bias experiences scales (transgender-related victimization, rejection, and discrimination) each include the phrase, "because of my gender identity or expression." Consequently, participants' perception of the role of their transgender identity and perception of perpetrators' motivations are critical to their responses and thus overall subscale scores. Non-affirmation items, on the other hand, measure a participant's current experience of having their gender identity disrespected and/or not recognized, and can be endorsed regardless of whether a participant would attribute their experiences directly to anti-transgender bias.

In discussing the development of the non-affirmation subscale, Testa and colleagues (2015) noted that this experience of not having "one's internal sense of gender identity...affirmed by others" (p. 66) is a minority stressor unique to transgender people (as opposed to other minority groups). Notably, there is a large gap in the literature with regard to gender affirmation, or lack thereof, and its role in transgender people's mental health. Researchers examining gender minority stressors as risk factors have largely focused on experiences of discrimination, rejection, and victimization (e.g., bullying, family support). In their validation study that first introduced the scale, Testa and colleagues (2015) found statistically significant correlations between non-affirmation and perceived life stress, perceived social support, belongingness, burdensomeness, symptoms of depression, and symptoms of social anxiety, but no other known quantitative work on non-affirmation has been published. By modeling non-affirmation 
as a distinct predictor (exogenous) variable, the current study is the first to my knowledge that examines the unique relationship of these experiences to trans people's mental health after controlling for anti-transgender bias experiences.

\section{High Rates of PTSD Symptoms and Trauma Exposure}

Before reviewing the model at the heart of my research questions, it is important to highlight the incredibly high rates of post-traumatic stress in my sample. Using the recommended cut-point for the overall measure, $46 \%$ of this sample reported clinically significant post-traumatic stress, and $44 \%$ of participants met criteria for a provisional diagnosis of PTSD based on DSM-5 symptom endorsement. The common endorsement of clinically significant PTSD symptoms in my sample is concerning. A prevalence rate of nearly $50 \%$ is incredibly high. As I discussed in my Methodology chapter, my sample should not be considered wholly representative of the transgender community, and thus the prevalence found should not be interpreted as accurate measures of the transgender population at large. However, they warrant examination with this limitation in mind, and importantly, the prevalence of PTSD in my sample is consistent with previous research with more diverse samples of transgender people (Reisner et al., 2016), suggesting it may not have been inflated by sampling. One other known study has examined post-traumatic stress rates in a transgender sample and found lower prevalence (Shipherd et al., 2011). Notably, that study used a small sample of transgender women and cross-dressing men that was $97 \%$ White and largely professional with higher socio-economic status (Shipherd et al., 2011). Given the facts that White people were still overrepresented in both my sample and Reisner and colleagues' (2016) sample, and that online samples tend to be underrepresentative of some of the most marginalized communities (e.g., homeless), it is possible that my study's rate of clinically significant post-traumatic stress 
is actually smaller than the prevalence found in the actual transgender community. However this lack of representation may be balanced by the use of some therapist-based recruitment efforts (discussed in Method chapter), which could have contributed to mild inflation of symptomatic reports in my study.

Note that a 2013 study of adults in the U.S. $(N=2,953)$ estimated that $3.8 \%$ met DSM-5 criteria for PTSD within the six months prior to participation (Kilatprick et al., 2013). A direct comparison with the frequency of a provisional PTSD diagnosis in the current sample is inappropriate, as Kilpatrick and colleagues' prevalence rate was not based solely on symptom endorsement; to meet criteria, those researchers required participants have exposure to a Criterion A defined trauma event and functional impairment, neither of which were evaluated in the current study. Still, the fact that nearly half of my participants indicated significant post-traumatic distress is alarming and likely indicates a probable and stark disparity between transgender and cisgender populations.

Additionally of note are the high rates of exposure to bias and potentially traumatic events. This is consistent with prior research (e.g., Grant et al., 2011; Reisner et al., 2016), and suggests that rejection, discrimination, victimization, and other traumas due to one's gender identity, expression, or history are endemic in transgender populations. A very small minority of participants in this study denied having experienced any bias or other traumatic event due to their gender.

\section{The Roles of Anti-Transgender Bias Experiences and Non-Affirmation in PTS}

The high rates of exposure and distress make understanding relationships and possible mechanisms all the more important. Returning to the main findings, structural equation modeling yielded a number of meaningful results regarding the possible roles of 
anti-transgender bias experiences and non-affirmation with post-traumatic stress. First, results demonstrated a statistically significant relationship between both anti-transgender bias experiences (which include the factors of transgender-related rejection, discrimination, and victimization) and non-affirmation and PTSD symptoms. Although this was partially mediated by internalized transphobia, $85 \%$ of each of the relationships was direct, indicating that while internalized transphobia is an important part of understanding how these factors relate to PTS, other mechanisms must be at play.

These findings lend support to the conceptualization of anti-transgender bias experiences and non-affirmation as potentially traumatic events themselves that can directly contribute to the development of post-traumatic stress symptoms. This interpretation is consistent with the feminist and multicultural models of trauma and posttraumatic stress explored in Chapter 1. Bias experience and non-affirmation could be conceptualized as violations of various security dimensions, as outlined in Chapter 1, such that they may not constitute physical threat to life but do constitute threats to one's sense of meaning in their own existence, sense of worth, sense of belonging, and sense of intimacy (or possibility of future intimacy). Similar to the ways in which racist experiences "violate one's existing way of making sense of self and the world, [creating] intense fear and destabilization" (McFarlane \& Girolama, 1996, p. 485, as quoted in Bryant-Davis \& Ocampo, 2005), anti-transgender bias experiences and particularly nonaffirmation experiences could have similar impacts on transgender people. Additionally, it cannot be ignored that while not always threats of direct physical violence, experiences of bias can threaten livelihoods/income, shelter, medical care, et cetera, and thus can be experienced as violations of even physical dimensions of security. These violations of security (psychological, interpersonal, and physical), sense of safety, and stability could 
explain increased risk of post-traumatic stress symptoms, which are typically considered to be maladaptive efforts at rebuilding safety in the aftermath of trauma (Briere \& Scott, 2015).

A key piece of understanding of the traumagenic nature of these oppression-based experiences is their chronicity and cumulative impact. To illustrate this, Brown (2008) uses the metaphor of very small drops of acid rain falling on stone, each drop with a different dilution but enough that it damages and thus etches the stone over time.

"Each drop by itself does little damage and may in fact etch the stone in such a way as to make it more beautiful. Thus, in some way the experience of daily microaggressions may evoke resilient coping responses. Yet each drop of emotion acid creates just enough damage to render the next drop more damaging. At times the dilution of the acid is such that the particular microaggression is barely perceived; at other times its sting is more apparent... Over time a fissure develops in the form of an emotional vulnerability that is invisible so long as certain aspects of the biopsychosocial and spiritual environment remain steady or supportive." (104)

Brown (2008) argues that symptoms only present "when enough acid has fallen or when the environment shifts sufficiently to open wide the crack." The relatively small effect size of the relationships found in this study is likely due in part to my inability to model protective factors or the supportive environments Brown (2008) references, due to the need for parsimony and statistical power.

Another way of interpreting these findings is to conceptualize anti-transgender bias experiences and non-affirmation as triggers. Triggers are "learned alarms" (Cahill \& 
Foa, 2001 p. 58), internal or external stimuli associated with an individual's trauma memory that when experienced signal threat to the individual or cue re-experiencing symptoms. Regular exposure to triggering events and stressors is associated with difficulty recovering from symptoms of PTSD (Raio, Brignon-Perez, Goldman, \& Phelps, 2015). Root (1992) described oppression-based experiences as insidious trauma that, in part due to their chronicity and cumulative nature, prevent marginalized individuals from forgetting their oppressed place in the world. Experiences like antitransgender bias events and non-affirmation may remind transgender people not only that they are devalued in society, but may also specifically call to memory (explicitly or implicitly) previous trauma and threats to safety.

Brown (2008) described oppression-based experiences as "the small but everpresent pulls of energy toward a survival level of consciousness, the reminders that someone somewhere is trying to make you and people like you less welcome on the planet" (p.103). Brown's sentiment could be restated using the cognitive-behavioral model of Emotion Processing Theory of PTSD (Foa \& Cahill, 2001): oppression-based experiences are stored within fear networks/emotion structures related to past traumatic experiences; accordingly, encounters that may hold little objective threat activate fullfledged threat responses associated with those past traumas. As an example, a transgender person might have been traumatically assaulted due to their gender identity or expression; years later, someone mistreating them due to their gender identity or expression (e.g., staring at them, using invalidating pronouns or a slur) may activate the fear network related to the traumatic assault. Given the ubiquity of anti-transgender bias experiences and non-affirmation in trans people's lives, one can assume that trans folks are being constantly triggered - or, in Brown's (2008) language, constantly pulled "toward a 
survival level of consciousness" (p. 103). Coupled with the high rates of exposure to even traditionally defined potentially traumatic events, this could explain high rates of posttraumatic distress. It is well-accepted across schools of PTSD etiology and recovery that one must have safety and stability in order to heal from past traumas (Briere \& Scott, 2015).

\section{The Mediating Role of Internalized Transphobia}

I also demonstrated that internalized transphobia is a relevant construct when discussing post-traumatic stress in transgender populations. Consistent with my hypotheses and Lee (2009) and Budden's (2009) models of shame-based PTSD in which attacks on the social self activate and/or contribute to shame-based understandings of traumatic events, internalized transphobia partially mediated the relationships between anti-transgender bias experiences and non-affirmation, and symptoms of PTSD. It explained approximately $15 \%$ of the relationships between anti-transgender bias and nonaffirmation and post-traumatic stress. This suggests that internalized transphobia may be one mechanism through which these relationships operate. As discussed in Chapter 1, I hypothesized the significance of this partial mediation largely due to literature that implicates shame and internalized stigma in the avoidance of adaptive processing of traumatic experiences. Although I did not measure avoidance, previous research that has demonstrated relationships between internalized homophobia and lesbians' PTSD symptom severity found experiential avoidance to be a significant mediator (Gold et al., 2009; Gold et al., 2011). I cannot make conclusions as to the role avoidance plays in my sample's symptoms, but I hypothesize that it is a major mechanism in the relationship found between internalized transphobia and PTSD symptom severity and worthy of further inquiry. It may also be that transgender people with higher levels of internalized 
transphobia are less likely to utilize coping strategies that rely on connection to the transgender community as a method of avoiding triggering a sense of shame (Lee, 2001). This could be impactful, as an emerging body of literature proposes that connection to, support from, and belonging within the transgender community are key factors in trans people's positive mental health outcomes (e.g., Austin \& Goodman, 2017; Barr et al., 2017; Nuttbrock et al., 2015; Pflum, Testa, Balsam, Goldblum, \& Bongar, 2015).

The significant relationship between internalized transphobia and both antitransgender bias experiences and non-affirmation can be understood through the process of internalizing external stigma (Herek, Gillis, \& Cogan, 2009). Transgender people who experience higher levels of anti-transgender bias and non-affirmation are experiencing increased levels of negative messages about being transgender. This is indeed a risk factor for integrating these messages into one's own worldview and view of oneself (Feinstein, Goldfried, \& Davila, 2012). Many theorists have proposed models for understanding the why and how of the internalization of these messages, which in many ways is beyond the scope of the current study's work and implications. It is, however, worth returning briefly to the Chapter 1 discussion of shame and internalized stigma in the aftermath of trauma: feminist models of post-traumatic stress posit that post-traumatic shame is related to the concept of unique vulnerability, which stated in simplified terms, is the idea that a person was victimized because of some set of characteristics unique to them (Brown, 2008; Herman, 1992; Root, 1992). Although the shame from this can prevent recovery, a survivor's perspective of unique vulnerability should also be understood as psychologically protective because it may provide them with a way to make meaning out of the disorienting trauma and/or because it may offer them a sense of control over their likelihood to be revictimized (Herman, 1992; Root, 1992). Internalizing 
negative messages about transgender people in the aftermath of anti-transgender bias or non-affirmation may protect transgender survivors against cognitive dissonance and a sense of lack of control, even as it increases the risk of experiencing post-traumatic stress. Notably the relationship between internalized transphobia and anti-transgender bias experiences and non-affirmation in this study was small, and there are likely many unmodeled factors that moderate it.

\section{Comparison of Non-Affirmation and Anti-Transgender Bias Experiences}

Non-affirmation had a stronger relationship with both internalized transphobia and PTSD symptom severity than anti-transgender experiences had. This is likely due to differences in measurement and the way the variables were entered into the model. In efforts to capture the chronicity of anti-transgender bias, psychologists who developed the subscales of transgender-related discrimination, victimization, and harassment utilized a unique set of response options: never; yes, before 18; yes, after 18; yes, in past year. Given that the frequency of these experiences is likely relevant to internalized transphobia and PTSD symptom severity, this way of scoring the measures may have attenuated the relationships I modeled. To illustrate, in my study a participant who experienced bias every day could report the same score as a person who experienced a bias event once in the past year. The non-affirmation measure on the other hand utilized a Likert-type response scale that captured the extent to which a person experienced nonaffirmation in their current lives. It is probable that if the variables were measured in similar ways, comparative strength of their relationships would yield different results.

Another methodological factor that might have contributed to the differences

found in parameter estimates is that non-affirmation was entered as an observed variable, while bias was entered a latent variable with the subscales of transgender-related 
discrimination, victimization, and rejection as its observed factors. The use of a latent variable allows for more parsing out of measurement error that is otherwise incorporated into path estimation. Given the likely contribution of methodological factors in the differential sizes of these relationships, theoretical conclusions or hypotheses about the ways in which bias experiences and non-affirmation differ in the model are not appropriate.

\section{Study Limitations and Future Research Directions}

An important limitation to this study is the lack of ability to model and measure intersectionality. Previous research has found that when transgender people attributed experiences of bias to a larger number of identities/experiences (for example, if a person experiences bias due to being transgender, being a racial minority, and having a disability), they endorse greater PTSD symptom severity than transgender people who endorse having experienced bias due to only one of their identities or experiences (Reisner et al., 2016). Because I evaluated bias exclusively related to being transgender, I cannot use these data to evaluate the interplay of transgender identity and other sources of oppression. Although this study offers important contributions to the understanding of transgender mental health, future research will be more valuable if it can incorporate analysis of intersecting oppressions and/or disparities.

Related, my sample was overrepresented by participants who identified as White and non-Hispanic (81\%), as well as individuals who were female-assigned at birth (72\%). It is worth considering whether the variables in this study and documented relationships operate differently within underrepresented groups - notably, transgender women of color. The fact that my findings are consistent with theory and existing literature suggests that they would also be found in a more representatively diverse transgender sample, but 
future studies with better representation and research that focuses exclusively on more marginalized members of the community will be necessary before we can fully understand the validity of my conclusions for the transgender community at large.

Additionally, there were some issues with measurement and that constitute limitations to the study. As discussed above, the response options for anti-transgender bias scales may not be the most effective way of evaluating the construct for purposes such as this study's. Efforts should be made at validating alternative scoring that would enable differentiation by frequency or severity of these bias experiences. This would be particularly useful in allowing for valid conclusions regarding comparisons between antitransgender bias and non-affirmation.

Finally, as is true of all cross-sectional research, results from this study cannot be used to draw conclusions about causality. Although theory allows us to hypothesize the directionality of the relationships we measured, this aspect of the model cannot be tested without longitudinal and/or experimental designs. Caution must be taken to not overstate these findings, and I encourage other researchers to develop longitudinal designs to more fully investigate the relationships I have identified. For example, if participants' data were gathered at multiple waves, exposure to bias and/or non-affirmation at an earlier time point could be evaluated for its ability to predict changes in internalized transphobia and post-traumatic stress at later time points.

\section{Clinical Implications}

By better understanding the role of bias experiences, non-affirmation, and internalized transphobia in trans people's mental health, specifically post-traumatic stress, clinicians will be better prepared and positioned to support transgender clients' psychological well-being and recovery from distress (Richmond et al., 2011; Richmond 
et al., 2017). In this section I will offer considerations for clinical work with transgender populations secondary to this study's conclusions by drawing on bodies of literature from the subfields of trauma treatment, gender and sexuality minority mental health, and racial minority mental health.

\section{An Important Note on Intersectionality}

As discussed in the above section on limitations, this study's conclusions are limited by the model's lack of attention to intersectional identities and experiences. Monolithic approaches to understanding transgender people's mental health needs risk further marginalizing individuals who experience oppression and reduced access to resources due to factors beyond transgender identity (e.g., transgender people of color; Singh et al., 2017). Indeed, a transgender person is never solely a transgender person: they are a transgender White man who grew up in poverty, or a transfeminine genderqueer Latina from a middle class background, or a transgender immigrant, or a transgender Christian older adult, and so forth. A person's experience of being transgender, and the experiences of bias, non-affirmation, and internalized transphobia will be shaped by their other identities, histories, and cultural contexts (Singh et al., 2017). It must be directly mentioned again that transgender people with marginalized intersecting identities (e.g., transgender people of color) face increased exposure to trauma, including bias, have reduced access to care, and have increased risk of poor mental and physical health outcomes (Budge, Thai, Tebbe, \& Howard, 2016; Lee, 2017; White Hughto, Murchison, Clark, Pachankis, \& Reisner, 2016). Many efforts that have attempted to increase clinicians' competence in working with gender diverse patients have not attended to the diversity within the population, thus decreasing the likelihood of applicability to the more marginalized members of the community (for example, 
transgender people of color, transgender people with non-Christian faith backgrounds, transgender people with disabilities, transgender people engaging in sex work; Singh et al., 2017). When considering the clinical implications of this work, I ask clinicians to be particularly mindful of the ways that intersecting identities may affect transgender people's experiences of bias and other trauma, affirmation, internalized transphobia, and post-traumatic stress. We as a field must also actively think about the ways marginalized intersecting identities and experiences may limit transgender people's access to resources and/or change the way they conceptualize, engage in, and respond to mental health helpseeking and treatment.

\section{Take Care to Do No Harm}

This study's finding that both non-affirmation and experiences of anti-transgender bias are directly and indirectly related to the severity of post-traumatic stress suggests multiple opportunities for interventions that would improve transgender people's mental health. The first is to reduce transgender people's experiences of non-affirmation and bias, which can begin with psychologists reducing the amount we as clinicians and researchers perpetrate non-affirmation and anti-transgender bias. There are multiple ethical arguments for such attention and effort, but given the international and nearuniversal commonality of the guiding principle of beneficence and nonmaleficence (American Psychological Association, 2017; Leach and Harbin, 1997) - considered by the International Union of Psychological Science (2008) to be an element of the principle of competent caring for the well-being of persons and peoples, I have chosen to particularly highlight this. Psychologists commit to have "active concern for the wellbeing of individuals, families, groups, and communities" and to "tak[e] care to do no harm to individuals, families, groups, and communities" (International Union of 
Psychological Psychologists, 2008; see also American Psychological Association, 2017). This study demonstrates that if we as clinicians expose transgender people to bias or nonaffirmation, we are increasing their risk of psychological distress - namely, posttraumatic stress; pairing these findings with theory, I argue that in such circumstances we are in fact contributing to distress. Thus in order to uphold our ethical duty, and likely our own compassion-driven moral commitments, we must be diligent in efforts to avoid being perpetrators of bias or non-affirmation against the trans community, particularly those under our care.

\section{Using Psychotherapy to Facilitate Recovery from and Resilience to Bias and Non-}

\section{Affirmation}

Internalized transphobia. In addition to avoiding perpetrating acts of bias or non-affirmation, my findings suggest that those tasked with supporting transgender people's mental health should facilitate recovery and healing from the impacts of antitransgender bias and non-affirmation. The mediating role of internalized transphobia indicates that attention to this construct and related distress in psychotherapy could be of benefit to clients experiencing bias-related post-traumatic stress. The literature on psychotherapy with transgender populations is limited, but a growing number of psychologists and clinician-researchers from other fields have discussed strategies for supporting the reduction of internalized transphobia and its impact on mental health. These strategies include a cognitive-behavioral model that focuses on psychoeducation about internalized transphobia and its origins in external stigma, as well as active challenging of negative thoughts about one's transgender identity and history and/or the transgender community (Austin \& Craig, 2015; Austin, Craig, \& Alessi, 2017). Clinicians alternatively working from a more psychodynamic perspective may be aided 
by McBee's (2013) conceptualization of work targeting internalized transphobia as work aimed at cohesion of self. In this approach, reduction in internalized transphobia is achieved through exploration of fragmented and disavowed pieces of the client's ego and/or history, which when done in an empathic and holding relational environment, allows a reconstruction of a cohesive narrative and unified sense of self (Borden, 2009; Fraser, 2009). Group psychotherapy is also emerging as a powerful medium for these interventions, as it allows for psychoeducation, self-exploration, and naming and challenging of internalized stigma in a context that inherently provides normalization. Group psychotherapy also facilitates connection to the transgender community, which has been consistently found to be related to better mental health in general (e.g., Barr et al., 2016) and decreased internalized transphobia specifically (dickey \& Loewy, 2010; Singh et al., 2011). Relatedly, liberation psychologists promote collective action (i.e., activism and advocacy for and within the trans community) as a way to reduce internalized transphobia (Puckett \& Levitt, 2015). Collective action is associated with lower levels of internalized transphobia, but some research has found that it is also related to increased experiences of anti-transgender bias and overall worse mental health (Breslow et al., 2015), so clinicians should be thoughtful in their encouragement of client involvement.

Bias and non-affirmation. Given the relatively small mediation role of internalized transphobia in the relationships between anti-transgender bias, nonaffirmation, and post-traumatic stress, clinicians who do not also directly address the impact of client experiences of bias and non-affirmation will likely miss important avenues for recovery and healing. My findings support Richmond and colleagues' (2012, 2017) call for thorough assessment of transgender clients' trauma histories and sequelae, 
including evaluation of the person's experience with bias and non-affirmation. This is consistent with a solidifying standard of best practice. In the sets of guidelines for working with transgender populations published by the American Psychological Association (2015) and the American Counseling Association (2009), authors call for specific attention to the impact of bias and stigma. Conceptualizing these experiences and the less often discussed experiences of non-affirmation as sources of trauma responses provides a helpful starting point in considering how to approach treatment that adequately addresses their impact. Richmond and colleagues (2012) recommend adapting models of trauma recovery for this work and specifically borrow from Herman's $(1992,2015)$ three-stage model of recovery from complex post-traumatic stress, in which safety and stability are first established - for example, through supporting desired steps in gender transitions to alleviate gender dysphoria and building helpful coping and self-care strategies (Richmond et al., 2012); then interventions focus on remembrance and mourning - this could also be considered the processing phase from Foa and Cahill's (2011) treatment model, discussed in Chapter 1; and finally on reconnection, which could involve efforts at collective action as described above (Richmond et al., 2012).

An area of clinical literature that is particularly relevant is that of treatment for racism-based post-traumatic stress, within which a growing number of psychologists have proposed models that range from broad approaches (Bryant-Davis \& Ocampo, 2007) highlighting various treatment focuses (e.g., acknowledgment, sharing, mourning, anger, self-care, coping, and resistance) to more specific strategies and protocols (e.g., Williams, Pena, \& Mier-Chairez, 2017; Williams et al., 2014). Although review of this literature is beyond the scope of the current study, future work adapting these models to 
be inclusive of anti-transgender bias and non-affirmation experiences would be of great benefit to the transgender and gender diverse community.

\section{Conclusion}

In reflecting on the various pathways for understanding the relationship between racism and post-traumatic stress, Ippen (2012) writes that "racism may (a) place individuals at risk for trauma exposure, (b) exacerbate the impact of trauma and increase the risk of impairment, and (c) be a form of psychological trauma in itself" (p. 42). These three options, not mutually exclusive, offer an effective frame for the conclusions of this study. This data showed that within a sample of transgender adults, anti-transgender bias experiences and non-affirmation are clearly related to post-traumatic stress. This sample, which notably is over represented by white transgender men (the least marginalized racial and gender categories within the trans community), also endorsed high rates of trauma and bias exposure. Contextualized within the theoretical frames offered by cognitivebehavioral, feminist, and multicultural models of trauma and PTSD, I conclude that antitransgender bias and non-affirmation may trigger trauma responses or, particularly in accumulation, be sources of traumatization themselves, echoing Ippen's (2012) eloquent statement above. While it appears that transgender people may have increased risk of trauma exposure than the general population given the high rates reported in this study, which is absolutely critical in understanding the increased risk of PTSD within the population, I found that exposure to these events alone is insufficient for understanding the relationship with post-traumatic stress.

In her seminal work on recovery from racist-based trauma, Thelma Bryant-Davis (2007) wrote that "healing requires recognition." Indeed two central themes to the work of trauma therapists are bearing witness to a survivor's story and naming trauma directly 
(Briere \& Scott, 2015; Herman, 2015; Herman \& Keane, 2012). As was reviewed in Chapter 1, determining which types of events the field names as traumatic has been a contentious part of the history of trauma psychology, which has often resulted in the dismissal and invalidation of survivors experiencing post-traumatic stress. It is my hope that this study will be a part of the other narrative in the field's history: that when members of marginalized communities are lifted up and listened to, their trauma is named, and the field adapts and progresses in response. My own transgender identity and history are not irrelevant to my work here. I have spent the past decade listening to my community's stories of the impact of oppression, while witnessing the ways my field dismissed trans people's suffering as purely pathological. As social disparities decrease and increasing numbers of transgender people enter the field of psychology, we will be carrying our community's stories with us, and I hope you will listen. Some of these stories - the ones I have chosen to focus on with this study - are stories of oppression and marginalization. This study highlights that the field of psychology must wrestle with the ways in which such experiences contribute to the high rates of traumatization amongst transgender people, particularly those with marginalized intersecting identities, like transgender women of color. Recognition - bearing witness and naming the impact - is the necessary foundation for any next steps, whether they be individual recovery and healing, community-based prevention, or a combination of the two. 


\section{REFERENCES}

Alessi, E. J., Martin, J. I. Gyamerha, A., \& Meyer, I. H. (2013). Prejudice events and traumatic stress among heterosexuals and lesbians, gay men and bisexuals. Journal of Aggression, Maltreatment, \& Trauma, 22. doi.org/10.1080/10926771.2013.785455

Allen, I. M. (1996). PTSD among African Americans. In A. J. Marsella, M. J. Friedman, E. T. Gerrity, \& R. M. Scurfield (Eds.), Ethnocultural aspects of posttraumatic stress disorder: Issues, research, and clinical applications (pp. 209-238). Washington, DC, US: American Psychological Association. http://dx.doi.org/10.1037/10555-008

American Psychiatric Association. (1980). Diagnostic and statistical manual of mental disorders, third edition (DSM-III). Washington DC: American Psychiatric Publishers.

American Psychiatric Association. (2000). Diagnostic and statistical manual of mental disorders, Fourth edition, revised (DSM-IV-R). Washington, DC: American Psychiatric Publishers.

American Psychiatric Association. (2013). Diagnostic and statistical manual of mental disorders, Fifth edition (DSM-5). Washington, DC: American Psychiatric Publishers.

American Psychological Association. (2015). Guidelines for psychological practice with transgender and gender nonconforming people. American Psychologist, 70, 832864. http://dx.doi.org/10.1037/a0039906 
American Psychological Association. (2017). Ethical principles of psychologists and code of conduct. Washington, DC: American Psychological Association. Accessed via https://www.apa.org/ethics/code/ethics-code-2017.pdf

Anderson, J. C., \& Gerbing, D. W. (1988). Structural equation modeling in practice: A review and recommended two-step approach. Psychological bulletin, 103(3), 411423.

Andrews, B., Brewin, C. R., Rose, S., \& Kirk, M. (2000). Predicting PTSD symptoms in victims of violent crime: The role of shame, anger, and childhood abuse. Journal of abnormal psychology, 109(1), 69.

Armour, C., Tsai, J., Durham, T. A., Charak, R., Biehn, T. L., Elhai, J. D., \& Pietrzak, R. H. (2015). Dimensional structure of DSM-5 posttraumatic stress symptoms: Support for a hybrid Anhedonia and Externalizing Behaviors model. Journal of psychiatric research, 61, 106-113. https://doi.org/10.1016/j.jpsychires.2014.10.012

Auer, M. K., Hohne, N., Bazarra-Castro, M. A. Ã. N., Pfister, H., Fuss, J., Stalla, G. K., et al. (2013). Psychopathological profiles in transsexuals and the challenge of their special status among the sexes. PLoS ONE, 8, 1-9. doi.org/10.1371/journal.pone.0078469

Austin, A., \& Craig, S. L. (2015). Transgender affirmative cognitive behavioral therapy: Clinical considerations and applications. Professional Psychology: Research and Practice, 46, 21-29. doi.org/10.1037/a0038642

Austin, A., \& Goodman, R. (2017). The impact of social connectedness and internalized transphobic stigma on self-esteem among transgender and gender non-conforming 
adults. Journal of homosexuality, 64(6), 825-841.

https://doi.org/10.1080/00918369.2016.1236587

Austin, A., Craig, S. L., \& Alessi, E. J. (2017). Affirmative cognitive behavior therapy with transgender and gender nonconforming adults. Psychiatric Clinics, 40(1), 141-156. https://doi.org/10.1016/j.psc.2016.10.003

Baranowsky, A. B., Young, M., Johnson-Douglas, S., Williams-Keeler, L., \& McCarrey, M. (1998). PTSD transmission: A review of secondary traumatization in Holocaust survivor families. Canadian Psychology/Psychologie canadienne, 39(4), 247-256. http://dx.doi.org/10.1037/h0086816

Barr, S. M., Budge, S. L., \& Adelson, J. L. (2016). Transgender community belongingness as a mediator between strength of transgender identity and wellbeing. Journal of Counseling Psychology, 63, 87-97. doi.org/10.1037/cou0000127

Barr, S. M., Budge, S. L., \& Adelson, J. L. (2016). Transgender community belongingness as a mediator between strength of transgender identity and wellbeing. Journal of Counseling Psychology, 63, 87-97. doi.org/10.1037/cou0000127

Bazargan, M., \& Galvan, F. (2012). Perceived discrimination and depression among lowincome Latina male-to-female transgender women. BMC Public Health, 12, 1-8. doi.org/10.1186/1471-2458-12-663

Beemyn, G., \& Rankin, S. (2011). The lives of transgender people. Columbia University Press. https://doi.org/10.1177/0049124187016001004

Bentler, P. M., \& Chou, C. P. (1987). Practical issues in structural modeling. Sociological Methods \& Research, 16(1), 78-117. 
Blanchard, E. B., Jones-Alexander, J., Buckley, T. C., \& Forneris, C. A. (1996).

Psychometric properties of the PTSD Checklist (PCL). Behaviour research and therapy, 34(8), 669-673. https://doi.org/10.1016/0005-7967(96)00033-2

Blevins, C. A., Weathers, F. W., Davis, M. T., Witte, T. K., \& Domino, J. L. (2015). The posttraumatic stress disorder checklist for DSM-5 (PCL-5): Development and initial psychometric evaluation. Journal of Traumatic Stress, 28(6), 489-498. https://doi.org/10.1002/jts.22059

Blosnich, J. R., Brown, G. R., Shipherd, PhD, J. C., Kauth, M., Piegari, R. I., \& Bossarte, R. M. (2013). Prevalence of gender identity disorder and suicide risk among transgender veterans utilizing Veterans Health Administration care. American Journal of Public Health, 103(10), e27-e32. http://doi.org/ 10.2105/AJPH.2013.301507

Bockting, W. (2015). Internalized transphobia. The international encyclopedia of human sexuality, 583-625.

Bockting, W. O., Coleman, E., \& Ettner, R. (2007). Developmental stages of the transgender coming out process: Toward an integrated identity. Handbook of Transgender Medicine and Surgery, 185-208.

Bockting, W. O., Knudson, G., \& Goldberg, J. M. (2006). Counseling and Mental Health Care for Transgender Adults and Loved Ones. International Journal of Transgenderism, 9(3-4), 35-82. http://doi.org/10.1300/J485v09n03_03

Bockting, W. O., Miner, M. H., Swinburne Romine, R. E., Hamilton, A., \& Coleman, E. (2013). Stigma, Mental Health, and Resilience in an Online Sample of the US Transgender Population. American Journal of Public Health, 103(5), 943-951. doi.org/10.2105/AJPH.2013.301241 
Bockting, W., \& Coleman, E. (2016). Developmental stages of the transgender comingout Process. Principles of Transgender Medicine and Surgery, 137.

Bollen, K. A. (1989). A new incremental fit index for general structural equation models. Sociological Methods \& Research, 17(3), 303-316. https://doi.org/10.1177/0049124189017003004

Borden, W. (2009). Contemporary psychodynamic theory and practice. Lyceum Books, Incorporated.

Bradford, J., Reisner, S. L., Honnold, J. A., \& Xavier, J. (2013). Experiences of transgender-related discrimination and implications for health: results from the Virginia Transgender Health Initiative Study. American journal of public health, 103(10), 1820-1829. http://doi.org/ 10.2105/AJPH.2012.300796

Brancu, M., Mann-Wrobel, M., Beckham, J. C., Wagner, H. R., Elliott, A., Robbins, A. T., ... \& Runnals, J. J. (2016). Subthreshold posttraumatic stress disorder: A metaanalytic review of DSM-IV prevalence and a proposed DSM-5 approach to measurement. Psychological trauma: theory, research, practice, and policy, 8(2), 222.

Breslau, N. (1998). Epidemiology of trauma and posttraumatic stress disorder. In R. Yehuda (Ed.), Review of psychiatry series. Psychological trauma (pp. 1-29). Arlington, VA, US: American Psychiatric Association.

Breslau, N., Chilcoat, H. D., Kessler, R. C., \& Davis, G. C. (1999). Previous exposure to trauma and PTSD effects of subsequent trauma: results from the Detroit Area Survey of Trauma. American journal of Psychiatry, 156(6), 902-907. https://doi.org/10.1176/ajp.156.6.902 
Breslow, A. S., Brewster, M. E., Velez, B. L., Wong, S., Geiger, E., \& Soderstrom, B. (2015). Resilience and collective action: Exploring buffers against minority stress for transgender individuals. Psychology of Sexual Orientation and Gender Diversity, 2(3), 253.

Breuer, J., \& Freud, S. (1895). Studies on hysteria. New York, NY: Basic Books, Inc. Accessed via https://archive.org/details/studiesonhysteri037649mbp

Briere, J. (2004). Psychological assessment of adult posttraumatic states:

Phenomenology, diagnosis, and measurement (2nd ed.). Washington, DC, US: American Psychological Association. http://dx.doi.org/10.1037/10809-000

Briere, J. N., \& Scott, C. (2014). Principles of trauma therapy: A guide to symptoms, evaluation, and treatment (DSM-5 update). Sage Publications.

Briere, J., \& Spinazzola, J. (2005). Phenomenology and psychological assessment of complex posttraumatic states. Journal of Traumatic Stress: Official Publication of The International Society for Traumatic Stress Studies, 18(5), 401-412. https://doi.org/10.1002/jts.20048

Brown, L. S. (2008). Cultural competence in trauma therapy: Beyond the flashback. Washington, DC, US: American Psychological Association. http://dx.doi.org/10.1037/11752-000

Bryant-Davis, T., \& Ocampo, C. (2006). A Therapeutic Approach to the Treatment of Racist-Incident-Based Trauma. Journal of Emotional Abuse, 6(4), 1-22. http://doi.org/10.1300/J135v06n04_01

Budden, A. (2009). The role of shame in posttraumatic stress disorder: A proposal for a socio-emotional model for DSM-V. Social Science \& Medicine, 69(7), 10321039. http://doi.org/10.1016/j.socscimed.20 
Budge, S. L., Adelson, J. L., \& Howard, K. A. S. (2013). Anxiety and depression in transgender individuals: The roles of transition status, loss, social support, and coping. Journal of Consulting and Clinical Psychology, 81(3), 545-557. http://doi.org/10.1037/a0031774

Budge, S. L., Barr, S. M., \& Keller, B. unpublished;

Budge, S. L., Thai, J. L., Tebbe, E. A., \& Howard, K. A. (2016). The intersection of race, sexual orientation, socioeconomic status, trans identity, and mental health outcomes. The Counseling Psychologist, 44(7), 1025-1049. https://doi.org/10.1177/0011000015609046

Burgess, A. W. (1983). Rape trauma syndrome. Behavioral Sciences \& the Law, 1(3), 97113. https://doi.org/10.1002/bs1.2370010310

Burgess, A. W., \& Holmstrom, L. L. (1974). Rape trauma syndrome. American journal of Psychiatry, 131(9), 981-986. https://doi.org/10.1176/ajp.131.9.981

Burham, K., \& Anderson, D. (2002). Model selection and multivariate inference: A practical information-theoretical approach. New York, NY: Springer.

Cahill, S. P., \& Foa, E. B. (2007). Psychological theories of PTSD. Handbook of PTSD: Science and practice, 55-77.

Campbell, D. G., Felker, B. L., Liu, C. F., Yano, E. M., Kirchner, J. E., Chan, D., ... \& Chaney, E. F. (2007). Prevalence of depression-PTSD comorbidity: Implications for clinical practice guidelines and primary care-based interventions. Journal of general internal medicine, 22(6), 711-718. https://doi.org/10.1007/s11606-0060101-4 
Carlson, E. B., \& Dalenberg, C. J. (2000). A conceptual framework for the impact of traumatic experiences. Trauma, Violence, \& Abuse, 1(1), 4-28. https://doi.org/10.1177/1524838000001001002

Carmel, T., Hopwood, R., \& Dickey, L. M. (2014). Mental health concerns. Trans bodies, trans selves: A resource guide for the transgender community, 305-332.

Carter, R. T., \& Helms, J. E. (2002). Racial discrimination and harassment: A race based traumatic stress disorder. American Forensic Examiners Conference, Orlando, FL.

Chou, T., Asnaani, A., \& Hofmann, S. G. (2012). Perception of racial discrimination and psychopathology across three US ethnic minority groups. Cultural Diversity and Ethnic Minority Psychology, 18(1), 74.

Clark, D. M., \& Wells, A. (1995). A cognitive model of social phobia. In R. G. Heimberg (Ed.) Social phobia: Diagnosis, assessment, and treatment, 69-93. Guilford Press.

Clements-Nolle, K., Marx, R., \& Katz, M. (2006). Attempted suicide among transgender persons: The influence of gender-based discrimination and victimization. Journal of homosexuality, 51(3), 53-69. https://doi.org/10.1300/J082v51n03_04

Crawford, R. (1977). You are dangerous to your health: the ideology and politics of victim blaming. International journal of health services, 7(4), 663-680. https://doi.org/10.2190/YU77-T7B1-EN9X-G0PN

Crocker, J., \& Major, B. (1989). Social stigma and self-esteem: The self-protective properties of stigma. Psychological review, 96(4), 608-630.

Davey, A., Bouman, W. P., Meyer, C., \& Arcelus, J. (2015). Interpersonal Functioning Among Treatment-Seeking Trans Individuals. Journal of Clinical Psychology, 71, 1173-1185. http://doi.org/10.1002/jclp.22209 
Dickey, L. M., \& Loewy, M. I. (2010). Group work with transgender clients. The Journal for Specialists in Group Work, 35(3), 236-245. https://doi.org/10.1080/01933922.2010.492904

dickey, L. M., Reisner, S. L., \& Juntunen, C. L. (2015). Non-suicidal self-injury in a large online sample of transgender adults. Professional Psychology: Research and Practice, 46, 3-11. http://doi.org/10.1037/a0038803

Dispenza, F., Watson, L. B., Chung, Y. B., \& Brack, G. (2012). Experience of careerrelated discrimination for female-to-male transgender persons: A qualitative study. The Career Development Quarterly, 60(1), 65-81. https://doi.org/10.1002/j.2161-0045.2012.00006.x

Ellis, B. H., MacDonald, H. Z., Lincoln, A. K., \& Cabral, H. J. (2008). Mental health of Somali adolescent refugees: The role of trauma, stress, and perceived discrimination. Journal of consulting and clinical psychology, 76(2), 184.

Epstein, S. (1991). The self-concept, the traumatic neurosis, and the structure of personality. In D. J. Ozer, J. M. Healy, Jr., \& A. J. Stewart (Eds.), Perspectives in personality, Vol. 3. Part A: Self and emotion; Part B: Approaches to understanding lives(pp. 63-98). London, England: Jessica Kingsley Publishers.

Erichsen, J. E. (1866). On railways and other injuries of the nervous system. Henry C. Lea. Accessed via https://archive.org/details/onrailwayandoth00ericgoog.

Erickson-Schroth, L. (2013). Update on the Biology of Transgender Identity. Journal of Gay \& Lesbian Mental Health, 17, 150-174. http://doi.org/10.1080/19359705.2013.753393

Erickson-Schroth, L. (Ed.). (2014). Trans bodies, trans selves: A resource for the transgender community. Oxford University Press. 
Fassin, D., \& Rechtman, R. (2009). The empire of trauma: An inquiry into the condition of victimhood. Princeton University Press.

Feinstein, B. A., Goldfried, M. R., \& Davila, J. (2012). The relationship between experiences of discrimination and mental health among lesbians and gay men: An examination of internalized homonegativity and rejection sensitivity as potential mechanisms. Journal of consulting and clinical psychology, 80(5), 917-927.

Fessler, D. (2007). From appeasement to conformity. In J. Tracy, R. Robins, \& J. Tangney (Eds.) Self-conscious emotions: Theory and research, 174-193. Guilford Press.

Fessler, D. M. (1999). Toward an understanding of the universality of second order emotions. In A. Hinton (Ed.) Biocultural approaches to the emotions, 75-116.

Field, A. (2009). Discovering statistics using SPSS. Sage publications.

Fisher, A. D., Bandini, E., Casale, H., Ferruccio, N., Meriggiola, M. C., Gualerzi, A., ... \& Stomaci, N. (2013). Sociodemographic and clinical features of gender identity disorder: an Italian multicentric evaluation. The journal of sexual medicine, 10(2), 408-419. https://doi.org/10.1111/j.1743-6109.2012.03006.x

Flores, A. R., Herman, J. L., Gates, G. J., \& Brown, T. N. T. (2016). How many adults identify as transgender in the United States? Los Angeles, CA: Williams Institute. https://williamsinstitute.law.ucla.edu/research/how-many-adults-identify-astransgender-in-the-united-states/

Foa, E. B. \& Cahill, S. P. (2001). Psychological therapies: Emotional processing. In N. J. Smelser \& B. Baltes (eds.), International Encyclopedia of the Social and Behavioral Sciences, 12363-12369. 
Foa, E. B., \& Kozak, M. J. (1985). Treatment of anxiety disorders: Implications for psychopathology. In A. H. Tuma \& J. D. Maser (Eds.), Anxiety and the anxiety disorders(pp. 421-452). Hillsdale, NJ, US: Lawrence Erlbaum Associates, Inc.

Foa, E. B., \& Kozak, M. J. (1986). Emotional processing of fear: exposure to corrective information. Psychological bulletin, 99(1), 20-35.

Foa, E. B., Hembree, E. A., \& Rothbaum, B. O. (2007). Prolonged exposure therapy for PTSD. New York: Oxford University.

Foa, E. B., Keane, T. M., \& Friedman, M. J. (2000). Guidelines for treatment of PTSD. Journal of traumatic stress, 13(4), 539-588. https://doi.org/10.1023/A:1007802031411

FORGE. (2005). Sexual Violence Research: Implications. FORGE. http://forgeforward.org/anti-violence/sexual-violence-research/implications/

Fraser, L. (2009). Depth psychotherapy with transgender people. Sexual and relationship therapy, 24(2), 126-142. https://doi.org/10.1080/14681990903003878

Fredriksen-Goldsen, K. I., Cook-Daniels, L., Kim, H. J., Erosheva, E. A., Emlet, C. A., Hoy-Ellis, C. P., ... \& Muraco, A. (2014). Physical and mental health of transgender older adults: An at-risk and underserved population. The Gerontologist, 54(3), 488-500. https://doi.org/10.1093/geront/gnt021

Friedman, M. J. (2014). A brief history of the PTSD diagnosis. Handbook of PTSD: Science and practice,

Friedman, M. J., Resick, P. A., \& Keane, T. M. (2014). PTSD from DSM-III to DSM-5: Progress and challenges. Handbook of PTSD: Science and practice, 3-20. 
Friedman, M. J., Resick, P. A., Bryant, R. A., \& Brewin, C. R. (2011). Considering PTSD for DSM-5. Depression and anxiety, 28(9), 750-769. https://doi.org/10.1002/da.20767

George, W. H., \& Martínez, L. J. (2002). Victim blaming in rape: Effects of victim and perpetrator race, type of rape, and participant racism. Psychology of Women Quarterly, 26(2), 110-119. https://doi.org/10.1111/1471-6402.00049

Gilbert, P. (1997). The evolution of social attractiveness and its role in shame, humiliation, guilt and therapy. British Journal of Medical Psychology, 70(2), 113147. https://doi.org/10.1111/j.2044-8341.1997.tb01893.x

Gilbert, P. (2000). The relationship of shame, social anxiety and depression: The role of the evaluation of social rank. Clinical Psychology \& Psychotherapy: An International Journal of Theory \& Practice, 7(3), 174-189. https://doi.org/10.1002/1099-0879(200007)7:3<174::AID-CPP236>3.0.CO;2-U Gilboa-Schechtman, E., \& Foa, E. B. (2001). Patterns of recovery from trauma: The use of intraindividual analysis. Journal of Abnormal Psychology, 110(3), 392.

Gillihan, S. J., Cahill, S. P., \& Foa, E. B. (2014). Psychological theories of PTSD. Handbook of PTSD: Science and practice, 166-184.

Goffman, E. (1963). Stigma. Notes on the Management of Spoiled Identity. New York: Simon and Shuster.

Gold, S. D., Dickstein, B. D., Marx, B. P., \& Lexington, J. M. (2009). Psychological outcomes among lesbian sexual assault survivors: An examination of internalized homophobia and experiential avoidance. Psychology of Women Quarterly, 33(1), 54-66. http://doi.org/10.1111/j.1471-6402.2008.01474.x 
Gold, S. D., Feinstein, B. A., Skidmore, W. C., \& Marx, B. P. (2011). Childhood physical abuse, internalized homophobia, and experiential avoidance among lesbians and gay men. Psychological Trauma: Theory, Research, Practice, and Policy, 3(1), 50-60. http://doi.org/10.1037/a0020487

Gold, S. D., Feinstein, B. A., Skidmore, W. C., \& Marx, B. P. (2011). Childhood physical abuse, internalized homophobia, and experiential avoidance among lesbians and gay men. Psychological Trauma: Theory, Research, Practice, and Policy, 3(1), 50-60. http://doi.org/10.1037/a0020487

Gold, S. D., Marx, B. P., Soler-Baillo, J. M., \& Sloan, D. M. (2005). Is life stress more traumatic than traumatic stress?. Journal of Anxiety Disorders, 19(6), 687-698. https://doi.org/10.1016/j.janxdis.2004.06.002

Goldblum, P., Testa, R. J., Pflum, S., Hendricks, M. L., Bradford, J., \& Bongar, B. (2012). The relationship between gender-based victimization and suicide attempts in transgender people. Professional Psychology: Research and Practice, 43(5), 468-475. http://doi.org/10.1037/a0029605

Grant, J. M., Mottet, L., Tanis, J. E., Harrison, J., Herman, J., \& Keisling, M. (2011). Injustice at every turn: A report of the national transgender discrimination survey. National Center for Transgender Equality.

Gravetter, F. J., \& Wallnau, L. B. (2016). Statistics for the behavioral sciences. Cengage Learning.

Grey, N., Holmes, E., \& Brewin, C. R. (2001). Peritraumatic emotional "hot spots" in memory. Behavioural and Cognitive Psychotherapy, 29(3), 367-372. https://doi.org/10.1017/S1352465801003095 
Haas, A. P., Rodgers, P. L., \& Herman, J. (2014). Suicide attempts among transgender and gender non-conforming adults: Findings of the National Transgender Discrimination Survey. American Foundation for Suicide Prevention. https://williamsinstitute.law.ucla.edu/wp-content/uploads/AFSP-WilliamsSuicide-Report-Final.pdf

Helms, J. E., Nicolas, G., \& Green, C. E. (2010). Racism and ethnoviolence as trauma: Enhancing professional training. Traumatology, 16(4), 53-62. http://doi.org/10.1177/1534765610389595

Hendricks, M. L., \& Testa, R. J. (2012). A conceptual framework for clinical work with transgender and gender nonconforming clients: An adaptation of the Minority Stress Model. Professional Psychology: Research and Practice, 43(5), 460-467. http://doi.org/10.1037/a0029597

Hepp, U., Kraemer, B., Schnyder, U., \& Miller, N. (2005). Psychiatric comorbidity in gender identity disorder. Journal of Psychosomatic Research, 58, 259-61. https://doi.org/10.1016/j.jpsychores.2004.08.010

Herek, G. M., Gillis, J. R., \& Cogan, J. C. (1999). Psychological sequelae of hate-crime victimization among lesbian, gay, and bisexual adults. Journal of consulting and clinical psychology, 67(6), 945-951. http://dx.doi.org/10.1037/0022$\underline{006 X .67 .6 .945}$

Herman, J. L. (1992). Complex PTSD: A syndrome in survivors of prolonged and repeated trauma. Journal of traumatic stress, 5(3), 377-391. https://doi.org/10.1002/jts.2490050305

Herman, J. L. (1997). Trauma and recovery: The aftermath of violence: From domestic abuse to political terror. New York, NY: Basic Books. 
Herman, J. L. (2015). Trauma and Recovery: The Aftermath of Violence--From Domestic Abuse to Political Terror, Second edition. Basic Books.

Herman, J. L., \& Keane, T. M. (2012). Foreword. In R.A. McMackin, E. Newman, J. M., Fogler, \& T. M. Keane (eds.) Trauma therapy in context: The science and craft of evidence-based practice. Washington, DC: American Psychological Association.

Horowitz, M. J. (1986). Stress-response syndromes: A review of posttraumatic and adjustment disorders. Psychiatric Services, 37(3), 241-249.

https://doi.org/10.1176/ps.37.3.241

Ippen, C. G. (2012). Integrating a diversity-informed approach into evidence-based practice. Trauma therapy in context: the science and craft of evidence-based practice, 29-50.

James, S. E., Herman, J. L., Rankin, S., Keisling, M., Mottet, L., \& Anafi, M. (2016). The Report of the 2015 U.S. Transgender Survey. Washington, DC: National Center for Transgender Equality.

Janoff-Bulman, R. (1979). Characterological versus behavioral self-blame: Inquiries into depression and rape. Journal of personality and social psychology, 37(10), 17981809. http://dx.doi.org/10.1037/0022-3514.37.10.1798

Janoff-Bulman, R. (1985). The aftermath of victimization: Rebuilding shattered assumptions. In C. R. Figley (ed.), Trauma and its wake, 15-35. New York, NY: Routledge.

Keane, T. M., Zimering, R. T., \& Caddell, J. M. (1985). A behavioral formulation of posttraumatic stress disorder in Vietnam veterans. Behavior Therapist, 8(1), 9-12.

Kenny, D. A. (2015). Measuring model fit. http://davidakenny.net/cm/fit.htm 
Keo-Meier, C. L., Herman, L. I., Reisner, S. L., Pardo, S. T., Sharp, C., \& Babcock, J. C. (2015). Testosterone treatment and MMPI-2 improvement in transgender men: A prospective controlled study. Journal of Consulting and Clinical Psychology, 83(1), 143-156. http://doi.org/10.1037/a0037599

Kessler, R. C., Sonnega, A., Bromet, E., Hughes, M., \& Nelson, C. B. (1995).

Posttraumatic stress disorder in the National Comorbidity Survey. Archives of general psychiatry, 52(12), 1048-1060.

doi:10.1001/archpsyc. 1995.03950240066012

Khan, L. (2011). Transgender health at the crossroads: legal norms, insurance markets, and the threat of healthcare reform. Yale J. Health Pol'y L. \& Ethics, 11, 375.

Kilpatrick, D. G., Resnick, H. S., Milanak, M. E., Miller, M. W., Keyes, K. M., \& Friedman, M. J. (2013). National estimates of exposure to traumatic events and PTSD prevalence using DSM-IV and DSM -5 criteria. Journal of traumatic stress, 26(5), 537-547. https://doi.org/10.1002/jts.21848

Kilpatrick, D. G., Veronen, L. J., \& Best, C. L. (1985). Factors predicting psychological distress among rape victims. Trauma and its Wake, 113-141.

Kilpatrick, D. G., Veronen, L. J., \& Resick, P. A. (1979). The aftermath of rape: Recent empirical findings. American Journal of Orthopsychiatry, 49(4), 658-669. http://dx.doi.org/10.1111/j.1939-0025.1979.tb02651.x

Kim, S., Thibodeau, R., \& Jorgensen, R. S. (2011). Shame, guilt, and depressive symptoms: A meta-analytic review. Psychological Bulletin, 137(1), 68-96. http://doi.org/10.1037/a0021466

Kira, I. A., Smith, I., Lewandowski, L., \& Templin, T. (2010). The effects of gender discrimination on refugee torture survivors: A cross-cultural traumatology 
perspective. Journal of the American Psychiatric Nurses Association, 16(5), 299306. https://doi.org/10.1177/1078390310384401

Kline, R. B. (2015). Principles and practice of structural equation modeling. Guilford publications.

Kubany, E. S., Leisen, M. B., Kaplan, A. S., Watson, S. B., Haynes, S. N., Owens, J. A., \& Burns, K. (2000). Development and preliminary validation of a brief broadspectrum measure of trauma exposure: the Traumatic Life Events Questionnaire. Psychological assessment, 12(2), 210-224.

Kulkarni, M., \& Pole, N. (2008). Psychiatric distress among Asian and European American survivors of the 1994 Northridge earthquake. The Journal of nervous and mental disease, 196(8), 597-604.

http://doi.org/10.1097/NMD.0b013e3181813290

Leach, M. M., \& Harbin, J. J. (1997). Psychological ethics codes: A comparison of twenty-four countries. International Journal of Psychology, 32(3), 181-192.

Lee, D. A. (2009). Compassion-focused cognitive therapy for shame-based trauma memories and flashbacks in post-traumatic stress disorder. A casebook of cognitive therapy for traumatic stress reactions, 230-246.

Lee, D. A., Scragg, P., \& Turner, S. (2001). The role of shame and guilt in traumatic events: A clinical model of shame-based and guilt-based PTSD. British Journal of Medical Psychology, 74(4), 451-466. http://doi.org/10.1348/000711201161109

Leskela, J., Dieperink, M., \& Thuras, P. (2002). Shame and Posttraumatic Stress Disorder. Journal of Traumatic Stress, 15(3), 223-226. http://doi.org/10.1023/A:1015255311837 
Lev, A. I. (2004). Transgender emergence: Therapeutic guidelines for working with transgender people and their families. Binghamton, NY: Haworth.

Levy, B. (2009). Stereotype embodiment: A psychosocial approach to aging. Current directions in psychological science, 18(6), 332-336. https://doi.org/10.1111/j.1467-8721.2009.01662.x

Levy, B. R., Slade, M. D., Murphy, T. E., \& Gill, T. M. (2012). Association between positive age stereotypes and recovery from disability in older persons. JAMA, 308(19), 1972-1973. http://doi.org/10.1001/jama.2012.14541

Libbrecht, K., \& Quackelbeen, J. (1995). On the early history of male hysteria and psychic trauma. Journal of the history of the behavioral sciences, 31(4), 370-384. https://doi.org/10.1002/1520-6696(199510)31:4<370::AIDJHBS2300310404>3.0.CO;2-6

Link, B. G., \& Phelan, J. C. (2001). Conceptualizing stigma. Annual review of Sociology, 27(1), 363-385. https://doi.org/10.1146/annurev.soc.27.1.363

Lissek, S., Rabin, S. J., McDowell, D. J., Dvir, S., Bradford, D. E., Geraci, M., ... \& Grillon, C. (2009). Impaired discriminative fear-conditioning resulting from elevated fear responding to learned safety cues among individuals with panic disorder. Behaviour research and therapy, 47(2), 111-118. https://doi.org/10.1016/j.brat.2008.10.017

Liu, P., Wang, L., Cao, C., Wang, R., Zhang, J., Zhang, B., ... \& Elhai, J. D. (2014). The underlying dimensions of DSM-5 posttraumatic stress disorder symptoms in an epidemiological sample of Chinese earthquake survivors. Journal of Anxiety Disorders, 28(4), 345-351. https://doi.org/10.1016/j.janxdis.2014.03.008 
Long, M. E., Elhai, J. D., Schweinle, A., Gray, M. J., Grubaugh, A. L., \& Frueh, B. C. (2008). Differences in posttraumatic stress disorder diagnostic rates and symptom severity between Criterion A1 and non-Criterion A1 stressors. Journal of anxiety disorders, 22(7), 1255-1263. https://doi.org/10.1016/j.janxdis.2008.01.006

McBee, C. (2013). Towards a more affirming perspective: Contemporary psychodynamic practice with trans* and gender non-conforming individuals. In The University of Chicago School of Social Service Administration (Org.), Advocates Forum (pp. 37-52).

McCann, I. L., \& Pearlman, L. A. (1990). Vicarious traumatization: A framework for understanding the psychological effects of working with victims. Journal of traumatic stress, 3(1), 131-149. https://doi.org/10.1007/BF00975140

McCoy, S. K., \& Major, B. (2003). Group Identification Moderates Emotional Responses to Perceived Prejudice. Personality and Social Psychology Bulletin, 29(8), 10051017. http://doi.org/10.1177/0146167203253466

McFall, L., \& Johnson, V. A. (2009). Shame: concept analysis. Journal of Theory Construction \& Testing, 13(2), 57-63.

Meier, S. C., Pardo, S. T., Labuski, C., \& Babcock, J. (2013). Measures of Clinical Health among Female-to-Male Transgender Persons as a Function of Sexual Orientation. Archives of Sexual Behavior, 42(3), 463-474.

http://doi.org/10.1007/s10508-012-0052-2

Meyer, I. H. (2003). Prejudice, social stress, and mental health in lesbian, gay, and bisexual populations: conceptual issues and research evidence. Psychological bulletin, 129(5), 674-697. 
Milad, M. R., Orr, S. P., Lasko, N. B., Chang, Y., Rauch, S. L., \& Pitman, R. K. (2008). Presence and acquired origin of reduced recall for fear extinction in PTSD: results of a twin study. Journal of psychiatric research, 42(7), 515-520.

Milad, M. R., Pitman, R. K., Ellis, C. B., Gold, A. L., Shin, L. M., Lasko, N. B., ... \& Rauch, S. L. (2009). Neurobiological basis of failure to recall extinction memory in posttraumatic stress disorder. Biological psychiatry, 66(12), 1075-1082.

Miner, M. H., Bockting, W. O., Romine, R. S., \& Raman, S. (2012). Conducting Internet research with the transgender population: Reaching broad samples and collecting valid data. Social Science Computer Review, 30(2), 202-211. https://doi.org/10.1177/0894439311404795

Mizock, L., \& Lewis, T. (2008). Trauma in Transgender Populations: Risk, Resilience, and Clinical Care. Journal of Emotional Abuse, 8(3), 335-354. http://doi.org/10.1080/10926790802262523

Mock, J. (2014). Redefining realness: My path to womanhood, identity, love \& so much more. Simon and Schuster.

Mol, S. S., Arntz, A., Metsemakers, J. F., Dinant, G. J., Vilters-van Montfort, P. A., \& Knottnerus, J. A. (2005). Symptoms of post-traumatic stress disorder after nontraumatic events: evidence from an open population study. The British Journal of Psychiatry, 186(6), 494-499. https://doi.org/10.1192/bjp.186.6.494

Monson, C. M., Friedman, M. J., \& La Bash, H. (2014). A psychological history of PTSD. In M. J. Friedman, T. M. Keane, \& P. A. Resick (eds.). Handbook of PTSD: Science and Practice (Second edition). Guilford Press.

Montgomery, D. C., Peck, E. A., \& Vining, G. G. (2013). Solutions manual to accompany introduction to linear regression analysis. John Wiley \& Sons. 
Morgan, S. W., \& Stevens, P. E. (2008). Transgender Identity Development as Represented by a Group of Female-to-Male Transgendered Adults. Issues in Mental Health Nursing, 29(6), 585-599. http://doi.org/10.1080/01612840802048782

Morgan, S. W., \& Stevens, P. E. (2012). Transgender Identity Development as Represented by a Group of Transgendered Adults. Issues in Mental Health Nursing, 33(5), 301-308. http://doi.org/10.3109/01612840.2011.653657

Mowrer, O. H. (1960). Learning theory and behavior. Hoboken, NJ, US: John Wiley \& Sons Inc. $\underline{\text { http://dx.doi.org/10.1037/10802-000 }}$

Nadal, K. L., Rivera, D. P., Corpus, J. H., \& Sue, D. W. (2010). Sexual orientation and transgender microaggressions. In D. W. Sue (ed.), Microaggressions and marginality: Manifestation, dynamics, and impact, 217-240. John Wiley \& Sons.

Nadal, K. L., Skolnik, A., \& Wong, Y. (2012). Interpersonal and Systemic Microaggressions Toward Transgender People: Implications for Counseling. Journal of LGBT Issues in Counseling, 6(1), 55-82.

http://doi.org/10.1080/15538605.2012.648583

National Center for PTSD. (2017). PTSD Checklist for DSM-5 (PCL-5). https://www.ptsd.va.gov/professional/assessment/adult-sr/ptsd-checklist.asp Nuttbrock, L., Bockting, W., Rosenblum, A., Hwahng, S., Mason, M., Macri, M., \& Becker, J. (2015). Transgender community involvement and the psychological impact of abuse among transgender women. Psychology of Sexual Orientation and Gender Diversity, 2(4), 386-390.

Nuttbrock, L., Hwahng, S., Bockting, W. O., Rosenblum, A., Mason, M., Macri, M., \& Becker, J. (2010). Psychiatric Impact of Gender-Related Abuse Across the Life 
Course of Male-to-Female Transgender Persons. Journal of Sex Research, 47(1), 12-23. http://doi.org/10.1080/00224490903062258

Øktedalen, T., Hagtvet, K. A., Hoffart, A., Langkaas, T. F., \& Smucker, M. (2014). The Trauma Related Shame Inventory: Measuring trauma-related shame among patients with PTSD. Journal of Psychopathology and Behavioral Assessment, 36(4), 600-615. https://doi.org/10.1007/s10862-014-9422-5

Osborne, J. W. (2013). Best practices in data cleaning: A complete guide to everything you need to do before and after collecting your data. Sage.

Page, H. W. (1883). Injuries of the spine and spinal cord without apparent mechanical lesion, and nervous shock: In their surgical and medico-legal aspects. J. \& A. Churchill. Accessed via https://archive.org/details/injuriesofspines00page

Paradies, Y., Ben, J., Denson, N., Elias, A., Priest, N., Pieterse, A., ... \& Gee, G. (2015). Racism as a determinant of health: a systematic review and meta-analysis. PloS one, 10(9), e0138511. https://doi.org/10.1371/journal.pone.0138511

Parson, E. R. (1985). Intercultural setting: Encountering Black Vietnam veterans. In S. M. Sonnenberg, A. S. Blank, \& J. A. Tablott (eds.) Trauma of War: Stress and Recovery in Viet Name Veterans.

Peirce, J. M., Burke, C. K., Stoller, K. B., Neufeld, K. J., \& Brooner, R. K. (2009). Assessing traumatic event exposure: comparing the Traumatic Life Events Questionnaire to the Structured Clinical Interview for DSM-IV. Psychological Assessment, 21(2), 210-218.

Pequegnat, W., Rosser, B. S., Bowen, A. M., Bull, S. S., DiClemente, R. J., Bockting, W. O., ... \& Konstan, J. (2007). Conducting Internet-based HIV/STD prevention 
survey research: considerations in design and evaluation. AIDS and

Behavior, 11(4), 505-521. https://doi.org/10.1007/s10461-006-9172-9

Perez-Brumer, A., Hatzenbuehler, M. L., Oldenburg, C. E., \& Bockting, W. O. (2015).

Individual- and Structural-Level Risk Factors for Suicide Attempts Among Transgender Adults. Behavioral Medicine, 41(3), 164-171.

http://doi.org/10.1080/08964289.2015.1028322

Perilla, J. L., Norris, F. H., \& Lavizzo, E. A. (2002). Ethnicity, culture, and disaster response: Identifying and explaining ethnic differences in PTSD six months after Hurricane Andrew. Journal of social and clinical psychology, 21(1), 20-45. https://doi.org/10.1521/jscp.21.1.20.22404

Pflum, S. R., Testa, R. J., Balsam, K. F., Goldblum, P. B., \& Bongar, B. (2015). Social support, trans community connectedness, and mental health symptoms among transgender and gender nonconforming adults. Psychology of sexual orientation and gender diversity, 2(3), 281-286.

Powers, M. B., Halpern, J. M., Ferenschak, M. P., Gillihan, S. J., \& Foa, E. B. (2010). A meta-analytic review of prolonged exposure for posttraumatic stress disorder. Clinical Psychology Review, 30(6), 635-641. http://doi.org/10.1016/j.cpr.2010.04.007

Puckett, J. A., \& Levitt, H. M. (2015). Internalized stigma within sexual and gender minorities: Change strategies and clinical implications. Journal of LGBT Issues in Counseling, 9(4), 329-349. https://doi.org/10.1080/15538605.2015.1112336

Raftery, A. E. (1995). Bayesian model selection in social research. Sociological methodology, 111-163. 
Raio, C. M., Brignoni-Perez, E., Goldman, R., \& Phelps, E. A. (2014). Acute stress impairs the retrieval of extinction memory in humans. Neurobiology of Learning and Memory, 112, 212-221. https://doi.org/10.1016/j.nlm.2014.01.015

Reisner, S. L., White Hughto, J. M., Gamarel, K. E., Keuroghlian, A. S., Mizock, L., \& Pachankis, J. E. (2016). Discriminatory Experiences Associated With Posttraumatic Stress Disorder Symptoms Among Transgender Adults. Journal of Counseling Psychology, 1-12. http://doi.org/10.1037/cou0000143

Resick, P. A., \& Schnicke, M. K. (1992). Cognitive processing therapy for sexual assault victims. Journal of consulting and clinical psychology, 60(5), 748-755.

Reynolds, M., \& Brewin, C. R. (1999). Intrusive memories in depression and posttraumatic stress disorder. Behaviour research and therapy, 37(3), 201-215. https://doi.org/10.1016/S0005-7967(98)00132-6

Richmond, K. A., Burnes, T., \& Carroll, K. (2012). Lost in trans-lation: Interpreting systems of trauma for transgender clients. Traumatology, 18(1), 45-57. http://doi.org/10.1177/1534765610396726

Richmond, K., Burnes, T. R., Singh, A. A., \& Ferrara, M. (2017). Assessment and treatment of trauma with TGNC clients: A feminist approach. Affirmative counseling and psychological practice with transgender and gender nonconforming clients, 191-212.

Ritsher, J. B., Otilingam, P. G., \& Grajales, M. (2003). Internalized stigma of mental illness: psychometric properties of a new measure. Psychiatry research, 121(1), 31-49. https://doi.org/10.1016/j.psychres.2003.08.008

Roberts, A. L., Gilman, S. E., Breslau, J., Breslau, N., \& Koenen, K. C. (2011). Race/ethnic differences in exposure to traumatic events, development of post- 
traumatic stress disorder, and treatment-seeking for post-traumatic stress disorder in the United States. Psychological medicine, 41(1), 71-83.

https://doi.org/10.1017/S0033291710000401

Root, M. P. (1992). Reconstructing the impact of trauma on personality. In Brown, L. S., \& Ballou, M. (eds.), Personality and psychopathology: Feminist reappraisals, 229-265. Guilford Press.

Root, M. P. (1992). Reconstructing the impact of trauma on personality. In Brown, L. S., \& Ballou, M. (eds.), Personality and psychopathology: Feminist reappraisals, 229-265. Guilford Press.

Rubinstein B.B. (1983) Freud's Early Theories of Hysteria. In: Cohen R.S., Laudan L. (eds) Physics, Philosophy and Psychoanalysis. Boston Studies in the Philosophy of Science, vol 76. Springer, Dordrecht

Sanchez-Hucles, J. V. (1999). Racism: Emotional abusiveness and psychological trauma for ethnic minorities. Journal of Emotional Abuse, 1(2), 69-87. https://doi.org/10.1300/J135v01n02_04

Sánchez, F. J., \& Vilain, E. (2009). Collective self-esteem as a coping resource for maleto-female transsexuals. Journal of Counseling Psychology, 56(1), 202-209. http://doi.org/10.1037/a0014573

Schmader, T., \& Lickel, B. (2006). The approach and avoidance function of guilt and shame emotions: Comparing reactions to self-caused and other-caused wrongdoing. Motivation and Emotion, 30(1), 42-55. https://doi.org/10.1007/s11031-006-9006-0

Sellers, R. M., \& Shelton, J. N. (2003). The role of racial identity in perceived racial discrimination. Journal of personality and social psychology, 84(5), 1079-1092. 
Shipherd, J. C., Maguen, S., Skidmore, W. C., \& Abramovitz, S. M. (2011). Potentially traumatic events in a transgender sample: Frequency and associated symptoms. Traumatology, 17(2), 56-67. http://doi.org/10.1177/1534765610395614

Singh, A. A., \& McKleroy, V. S. (2011). “Just Getting Out of Bed Is a Revolutionary Act": The Resilience of Transgender People of Color Who Have Survived Traumatic Life Events. Traumatology, 17(2), 34-44. http://doi.org/10.1177/1534765610369261

Singh, A. A., \& McKleroy, V. S. (2011). “Just Getting Out of Bed Is a Revolutionary Act": The Resilience of Transgender People of Color Who Have Survived Traumatic Life Events. Traumatology, 17(2), 34-44. http://doi.org/10.1177/1534765610369261

Singh, A. A., Hays, D. G., \& Watson, L. S. (2011). Strength in the Face of Adversity: Resilience Strategies of Transgender Individuals. Journal of Counseling \& Development, 89(1), 20-27. http://doi.org/10.1002/j.1556-6678.2011.tb00057.x

Singh, A. A., Hwahng, S. J., Chang, S. C., \& White, B. (2017). Affirmative counseling with trans/gender-variant people of color. Trans-affirmative counseling and psychological practice, 41-68. Washington, DC: American Psychological Association.

Soper, D. (2016). A priori sample size calculator for structural equation models. https://www.danielsoper.com/statcalc/calculator.aspx?id=89

Spinazzola, J., Blaustein, M., \& Van Der Kolk, B. A. (2005). Posttraumatic stress disorder treatment outcome research: The study of unrepresentative samples?. Journal of Traumatic Stress: Official Publication of The International 
Society for Traumatic Stress Studies, 18(5), 425-436.

https://doi.org/10.1002/jts.20050

Szymanski, D. M., \& Balsam, K. F. (2011). Insidious trauma: Examining the relationship between heterosexism and lesbians' PTSD symptoms. Traumatology, 17(2), 4-13. http://doi.org/10.1177/1534765609358464

Tangney, J. P., \& Dearing, R. L. (2002). Emotions and social behavior. Shame and guilt. New York, NY: Guilford Press.

Tangney, J. P., Wagner, P., \& Gramzow, R. (1992). Proneness to shame, proneness to guilt, and psychopathology. ... Of Abnormal Psychology.

Terada, S., Matsumoto, Y., Sato, T., Okabe, N., Kishimoto, Y., \& Uchitomi, Y. (2012). Factors predicting psychiatric co-morbidity in gender-dysphoric adults. Psychiatry Research, 200(2-3), 469-474. http://doi.org/10.1016/j.psychres.2012.07.018

Testa, R. J., Habarth, J., Peta, J., Balsam, K., \& Bockting, W. O. (2015). Development of the Gender Minority Stress and Resilience Measure. Psychology of Sexual Orientation and Gender Diversity, 2(1), 65-77. http://doi.org/10.1037/sgd0000081

Testa, R. J., Jimenez, C. L., \& Rankin, S. S. (2013). Risk and Resilience During Transgender Identity Development: The Effects of Awareness and Engagement with Other Transgender People on Affect. Journal of Gay \& Lesbian Mental Health, 130806075543008. http://doi.org/10.1080/19359705.2013.805177

Testa, R. J., Sciacca, L. M., Wang, F., Hendricks, M. L., Goldblum, P., Bradford, J., \& Bongar, B. (2012). Effects of violence on transgender people. Professional 
Psychology: Research and Practice, 43(5), 452-459.

http://doi.org/10.1037/a0029604

U.S. Department of State. (undated). Gender designation change.

https://travel.state.gov/content/travel/en/passports/apply-renew-

passport/gender.html

van der Kolk, B. A. (2007). The history of trauma in psychiatry. In M. J. Friedman, T. M.

Keane, \& P. A. Resick (Eds.), Handbook of PTSD: Science and practice (pp. 19-

36). New York, NY, US: Guilford Press.

Van Hooff, M., McFarlane, A. C., Baur, J., Abraham, M., \& Barnes, D. J. (2009). The stressor Criterion-A1 and PTSD: A matter of opinion?. Journal of Anxiety Disorders, 23(1), 77-86. https://doi.org/10.1016/j.janxdis.2008.04.001

Vardeman, S. B., \& Morris, M. D. (2003). Statistics and ethics: some advice for young statisticians. The American Statistician, 57(1), 21-26.

https://doi.org/10.1198/0003130031072

Weathers, F. W., \& Keane, T. M. (2007). The Criterion A problem revisited:

Controversies and challenges in defining and measuring psychological

trauma. Journal of traumatic stress, 20(2), 107-121.

https://doi.org/10.1002/jts.20210

Weathers, F. W., Litz, B. T., Keane, T. M., Palmieri, P. A., Marx, B. P., \& Schnurr, P. P. (2013). The PTSD checklist for DSM-5 (PCL-5). Scale available from the National Center for PTSD at www.ptsd.va.gov.

Weisaeth, L. (2002). The European history of psychotraumatology. Journal of Traumatic Stress: Official Publication of The International Society for Traumatic Stress Studies, 15(6), 443-452. https://doi.org/10.1023/A:1020909620364 
Weisaeth, L. (2014). The history of psychic trauma. Handbook of PTSD-Science and practice, $38-59$.

Westerlund, E. (1986). Freud on sexual trauma: An historical review of seduction and betrayal. Psychology of Women Quarterly, 10(4), 297-310. https://doi.org/10.1111/j.1471-6402.1986.tb00755.x

White Hughto, J. M., Murchison, G. R., Clark, K., Pachankis, J. E., \& Reisner, S. L. (2016). Geographic and individual differences in healthcare access for US transgender adults: a multilevel analysis. LGBT health, 3(6), 424-433. https://doi.org/10.1089/lgbt.2016.0044

Williams, M. T., Malcoun, E., Sawyer, B. A., Davis, D. M., Nouri, L. B., \& Bruce, S. L. (2014). Cultural adaptations of prolonged exposure therapy for treatment and prevention of posttraumatic stress disorder in African Americans. Behavioral Sciences, 4(2), 102-124. doi:10.3390/bs4020102

Williams, M. T., Peña, A., \& Mier-Chairez, J. (2017). Tools for Assessing RacismRelated Stress and Trauma Among Latinos. In Toolkit for Counseling SpanishSpeaking Clients (pp. 71-95). Springer, Cham.

Willness, C. R., Steel, P., \& Lee, K. (2007). A meta-analysis of the antecedents and consequences of workplace sexual harassment. Personnel psychology, 60(1), 127162. https://doi.org/10.1111/j.1744-6570.2007.00067.x

Wolf, E. J., Harrington, K. M., Clark, S. L., \& Miller, M. W. (2013). Sample size requirements for structural equation models: An evaluation of power, bias, and solution propriety. Educational and psychological measurement, 73(6), 913-934. https://dx.doi.org/10.1177\%2F0013164413495237 
Wyatt, G. E. (1990). Sexual abuse of ethnic minority children: Identifying dimensions of victimization. Professional Psychology: Research and Practice, 21(5), 338-343. http://dx.doi.org/10.1037/0735-7028.21.5.338

Yealland, L. R. (1918). Hysterical disorders of warfare. Accessed via https://archive.org/details/hystericaldisord00yealuoft

Yehuda, R., \& LeDoux, J. (2007). Response variation following trauma: a translational neuroscience approach to understanding PTSD. Neuron, 56(1), 19-32. https://doi.org/10.1016/j.neuron.2007.09.006

Zimmerman, M., \& Mattia, J. I. (1999). Axis I diagnostic comorbidity and borderline personality disorder. Comprehensive psychiatry, 40(4), 245-252.

https://doi.org/10.1016/S0010-440X(99)90123-2 


\section{APPENDIX A}

Table 8

Variable Means

\begin{tabular}{lcclcl}
\hline Variable & $n$ & Min & Max & $M$ & $S D$ \\
\hline Discrimination & 525 & 0.00 & 15.00 & 4.21 & 2.94 \\
Rejection & 532 & 0.00 & 18.00 & 5.91 & 4.12 \\
Victimization & 541 & 0.00 & 17.00 & 3.10 & 3.22 \\
Bias Total & 512 & 0.00 & 49.00 & 13.16 & 8.92 \\
Non-Affirmation & 564 & 0.00 & 24.00 & 13.38 & 7.53 \\
Internalized Transphobia & 556 & 0.00 & 32.00 & 13.79 & 8.19 \\
Unrelated TLEQ & 540 & 0.00 & 98.00 & 16.86 & 15.49 \\
PCL-5: Re-experiencing & 513 & 0.00 & 20.00 & 6.67 & 5.56 \\
PCL-5: Avoidance & 515 & 0.00 & 8.00 & 3.49 & 2.74 \\
PCL-5: Negative Affect & 516 & 0.00 & 16.00 & 6.41 & 4.80 \\
PCL-5: Anhedonia & 516 & 0.00 & 12.00 & 4.88 & 3.85 \\
PCL-5: Externalizing Behavior & 516 & 0.00 & 8.00 & 1.94 & 2.08 \\
PCL-5: Anxious Arousal & 518 & 0.00 & 8.00 & 2.93 & 2.69 \\
PCL-5: Dysphoric Arousal & 516 & 0.00 & 8.00 & 3.74 & 2.63 \\
PCL-5: Total & 519 & 0.00 & 80.00 & 29.99 & 20.77 \\
\hline
\end{tabular}

Table 9

Estimated Correlation Matrix for Latent Variables

\begin{tabular}{lccccc}
\hline & 1 & 2 & 3 & 4 & 5 \\
\hline 1. Bias Experience & 1.00 & & & & \\
2. Non-Affirmation & $.17^{*}$ & 1.00 & & & \\
3. Internalized Transphobia & $.22^{*}$ & $.25^{*}$ & 1.00 & & \\
4. Unrelated TLEQ & $.37^{*}$ & .06 & $.08^{*}$ & 1.00 & \\
5. PTSD Symptom Severity & $.40^{*}$ & $.36^{*}$ & $.34^{*}$ & $.48^{*}$ & 1.00 \\
\hline$* p<.001$ & & & & &
\end{tabular}


Sebastian Mitchell Barr, Ph.D.

\section{CURRICULUM VITAE}

Psychology Fellow

Program for Psychotherapy

Cambridge Health Alliance / Harvard Medical School

sbarr@challiance.org

617-575-5839

\section{EDUCATION}

Ph.D. Counseling Psychology, 2018 (Anticipated)

University of Louisville

Dissertation Title: Understanding the relationships between anti-transgender

bias and post-traumatic stress (PTS): A model of internalized transphobiamediated PTS

Dissertation Chairs: Kate Snyder, Ph.D., \& Mark Leach, Ph.D.

Defended, October 2018

B.A. Psychology, 2010

Smith College

Clinical Training

August 2018 - August 2020 Post-Doctoral Fellow

Program for Psychotherapy

Cambridge Health Alliance / Harvard Med. School

Training Director: Marla Eby, Ph.D.

June 2017 - June $2018 \quad$ Pre-Doctoral Psychology Intern

Cambridge Health Alliance / Harvard Med. School

Training Director: Patricia Harney, Ph.D.

Pre-Doctoral Clinical Practica Experience

Aug 2016 - May 2017

Clinician, Trauma-Focused Psychotherapy

VHA Lexington - PTSD Clinical Team

Supervisor: Eric Russ, Ph.D.

Sept 2015-May 2016

Clinician, Gender-Affirming Psychotherapy 
Compass Counseling and Psych. Services, PLLC

Supervisor: Amy Greenamyer, Ph.D.

Sept 2015-May 2016

Neuropsychological Assessment Clinician Compass Counseling and Psych. Services, PLLC

Supervisor: Greg Perri, Psy.D.

Aug 2015-May 2016

Outreach Coordinator

University of Louisville Counseling Center

Supervisors: Aesha Tyler, Ph.D., \& Ruby Casiano, Ph.D.

Jul 2015-Sept 2015

Assessment Clinician

Brighter Futures, PLLC

Supervisors: Nick Watters, Ph.D., \& C.J. Quick, LPCC

May 2015-Sept 2015

Clinician, Gender-Affirming Group Psychotherapy

Private Practice

Supervisor: Joanna Morse, Psy.D.

May 2014-May 2015

Clinician, Child and Family Psychotherapy

Seven Counties Services - Transitions Trauma Team

Supervisors: Jessica Mattingly, Psy.D., \& David Finke,

Ph.D.

Sept 2013-May 2015

Clinician, Trauma-Focused Psychotherapy University of Louisville Depression Center

Supervisor: Eric Russ, Ph.D.

\section{Publications}

Puckett, J. A., Barr, S. M., Wadsworth, L. P., \& Thai, J. L. (2018). Considerations for clinical work and research with transgender and gender diverse individuals. Behavior Therapist, 41 (5), 253-263. http://www.abct.org/docs/PastIssue/41n5.pdf

Snyder, K. E., Barr, S. M., Honken, N. B., Heuser, A. R., \& Ralston, P. (2018). Navigating the first semester: An exploration of short-term changes in motivational beliefs among engineering undergraduates. Journal of Engineering Education, 107, 11-29. doi: 10.1002/jee.20187

Gabel, C.P., Cuesta-Vargas, A., Barr, S.M., Black, S.W., Osborne, J.W., \& Melloh, M. (2016). Confirmatory Factor Analysis of the Neck Disability Scale, comparing patients with whiplash associated disorders to a control group with nonspecific neck pain. European Spine Journal. doi: 10.1007/s00586-016-4543-z 
Barr, S.M., Budge, S.L., Adelson, J. (2016). Transgender belongingness as a mediator between strength of transgender identity and well-being. Journal of Counseling Psychology, 63, 87-97. doi: 10.1037/cou0000127

Bryan, J., Barr, S.M., Overtree, C., \& Mangine, J. (2015). Letting go of the binary: Comparing categorical and continuous measures of sex, gender, and sexuality [White paper].

\section{MANUSCRIPTS IN PREPARATION AND UNDER REVIEW}

Barr, S.M., Thai, J.L., \& Keo-Meier, C. (in review). Examining White and POC transgender men's non-linear decrease in depression, anxiety, and stress symptoms across the first year of testosterone treatment.

Barr, S.M., Rosenkrantz, D.E., \& Budge, S.L. (in preparation). Psychometric properties of the Objectified Body Consciousness Scale in a sample of transgender women.

\section{ACAdemic Conference Presentations}

Barr, S.M. 2018. Non-affirmation and exposure to anti-transgender bias related to increased severity of PTSD symptoms: Evaluating a model of partial mediation by internalized transphobia. In J. Shipherd (Discussant), Understanding the experience of trauma and minority stress in lesbian, gay, bisexual, and transgender populations: Implications for conceptualizations, practice, and policy. Symposium to be presented at the International Society for Traumatic Stress Studies Annual Meeting, Washington, D.C.

Barr, S.M., Thai, J.L., Adelson, J., \& Keo-Meier, C. (2018, April). Psychological effects of testosterone therapy on transgender men: Examining racial differences across first 12 months. Poster presented at Harvard Medical School Mysell Psychiatry Research Day, Boston, MA.

Hayes-Skelton, S., Barr, S.M., Montoya, H., Puckett, J.A., \& Wadsworth, L.P. (2018, April). Enhancing the cultural sensitivity of psychosocial interventions for anxiety and depression when working with LGBTQ+ clients. Roundtable presented at the ADAA Anxiety and Depression Conference, Washington, D.C.

Barr, S.M., Budge, S.L., \& Snyder, K. (2017, February). Understanding transgender men's experiences of bias through a framework of trauma and internalized transphobia: Results of an SEM mediation analysis. In S. Budge (Discussant), Recent research on transgender men's psychological distress: Focusing on risk factors, protective factors, and impact of gender transition. Symposium presented at United States Professional Association of Transgender Health (USPATH) Scientific Conference, Los Angeles, CA. 
Barr, S.M., Thai, J.L., \& Keo-Meier, C. (2017, February). Examining White and POC transgender men's non-linear decrease in depression, anxiety, and stress symptoms across the first year of testosterone treatment. In S. Budge (Discussant), Recent research on transgender men's psychological distress: Focusing on risk factors, protective factors, and impact of gender transition. Symposium presented at USPATH Scientific Conference, Los Angeles, CA.

Barr, S.M., \& Budge, S.L. (2016, August). Experiences of self-esteem and well-being for individuals with non-binary gender identities. In S. Chang (Discussant), Identity processes, well-being, and emotional processes for individuals with non-binary identities. Symposium presented at the annual meeting of the American Psychological Association, Denver, CO.

Rosenkrantz, D.E., \& Barr, S.M. (2016, August). Validating the subscales of the Objectified Body Consciousness Scale in a population of trans women. In Ruth E. Fassinger (Discussant), Emerging areas of science, practice, and education with LGBTQ women. Symposium presented at the annual meeting of the American Psychological Association, Denver, CO.

Barr, S.M. \& Roane, S.J. (2016, June). Effectively addressing comorbid issues in affirmative therapy with trans clients. Workshop presented as part of the professional training track at the Philadelphia Trans Health Conference. Philadelphia, PA.

Snyder, K. E., Barr, S. M., Honken, N. B., Heuser, A. R., \& Ralston, P. (2016, April). Navigating the first semester: An exploration of short-term changes in motivational beliefs among engineering undergraduates. Poster presented at the annual meeting of the American Educational Research Association, Washington, D.C.

Barr, S.M., \& Budge, S.L. (2014, August). Predictors and outcomes of community belongingness in a trans* population. Poster presented at the annual meeting of the American Psychological Association, Washington, D.C.

Keller, B.L., Barr, S.M., \& Budge, S.L. (2014, August). "For every bad, there's 40 good things that happen": A qualitative approach to understanding the positive emotional experiences of trans* women. In l. dickey (Discussant), The other side of the story: Trans* individuals' experiences of positivity and resilience. Symposium presented at the meeting of the American Psychological Association, Washington, D.C.

Carlton, F.J., Snyder, K.E., Barr, S.M., \& Patall, E.A. (2014, April). Everything and the kitchen sink: A meta-analytic review of interventions for academically underachieving students. Paper presented at the American Educational Research Association Annual Conference, Philadelphia, PA. 
Budge, S.L., \& Barr, S.M. (October, 2013). Addressing grief and role transitions for transgender clients experiencing gender identity incongruence. Paper presented at the Biennial North American Society for Psychotherapy Research Conference, Nashville, TN.

Barr, S.M., \& Budge, S.L. (2013, June). The role of identity integration in the emotional well-being of post-transition individuals. In WPATH graduate research symposium. Symposium presented at the Philadelphia Trans Health Conference, Philadelphia, PA.

_ (2013, March). Paper presented at Spring Research Conference, Lexington, KY.

Budge, S.L., Barr, S.M., Katz-Wise, S.L., Keller, B.L., \& Manthos, M. (2013, June). Incorporating positivity into psychotherapy with trans clients. Workshop presented at the Annual Philadelphia Transgender Health Conference, Philadelphia, PA.

Budge, S.L., \& Barr, S.M. (2013, April). Emotional and identity processes of trans* youth: A developmental approach. Paper presented at the Biennial Meeting of the Society for Research on Child Development, Seattle, WA.

Keller, B.L., Barr, S.M., \& Budge, S. L. (2013, March). “For every bad, there's 40 good things that happen": A qualitative approach to understanding the positive emotional experiences of trans* women. Poster presented at the Kentucky Psychological Association Student Research Conference, Louisville, KY.

Barr, S. M., Stahl, A., Manthos, M., \& Budge, S.L. (2012, November). "It means there aren't any rules and you don't have to ascribe to a specific binary": A qualitative examination of genderqueer identity. Paper presented at the Chicago LGBTQ Health \& Wellness Conference, Chicago, IL.

Budge, S.L., Barr, S.M., Orovecz, J., \& Rossman, H.K. (2012, November). Clinical work with LGBT youth. Workshop presented at the Annual Kentucky Psychological Association Conference, Louisville, KY.

\section{Invited Outreach And Professional Presentations}

Barr, S.M. (2019, April). Effective and affirming clinical work with transgender and gender diverse young adults. Workshop to be presented at Treating Young Adults: Mental Health Risks and Challenges offered by Harvard Medical School and Cambridge Health Alliance Department of Psychiatry, Boston, MA. 
Barr, S.M. (2018, November). Considerations for clinical work with transgender and gender diverse students. Invited professional development workshop at American University Counseling Services, Washington, D.C.

Barr, S.M. (2018, February). Considerations for clinical work with transgender and gender diverse populations. Grand Rounds presented at Cambridge Health Alliance / Harvard Medical School, Cambridge, MA.

Eleazer, J.R., Roane, S.J., \& Barr, S.M. (2016, July). Affirmative treatment for transgender service members in military mental health. Workshop presented to Army behavioral health providers, Ft. Knox, KY.

Barr, S.M., \& Barr, K.R. (2016, July). Introduction to transgender youth and their needs in a primary care setting. Invited presentation to doctors, nurses, and staff, Novant Health - Steelecroft Pediatrics, Charlotte, NC.

Barr, S.M. (2016, March). Transgender identity and mental health. Invited presentation to clinicians, trainees, and staff, University of Louisville Counseling Center, Louisville, KY.

Barr, S.M. (2016, February). Emotions and your mental health. Invited presentation to undergraduate students, University of Louisville Resident Student Association meeting, Louisville, KY.

Barr, S.M. (2015, January). Coping 101: A primer on helpful coping for trans youth. Workshop presented to trans youth and mentors, Louisville Youth Group, Louisville, KY.

Barr, S.M. (2015, June). A review of trans psychology and mental health. Invited presentation to mental health clinicians, medical healthcare providers, transgender community members, and allies, Charlotte, NC.

Barr, S.M. (2015, April). Risk and resilience: The mental health of trans and gender non-conforming individuals. Invited presentation to undergraduate students, University of Louisville Psi Chi chapter meeting, Louisville, KY.

Russ, E., \& Barr, S.M. (2014, April). Current perspectives on trauma and PTSD: DSM5 , personality, and implications for psychotherapy. Invited presentation to veterans and service providers, Operation Headed Home, Somerset, KY.

Barr, S.M., Eleazer, J., Nguyen, Y., \& Budge, S.L. (2014, January). “I just want to deal with it on my own": Learning helpful coping strategies related to trans* stressors. Workshop presented to active duty transgender services members at a confidential conference. 
Budge, S.L., Barr, S.M., \& Orovecz, J. (2013, April). LGBTQ+ identities in college. Invited presentation to undergraduate students, graduate students, faculty, and staff, Georgetown College, Georgetown, KY.

Snyder, K.E. \& Barr, S.M. (2013, January). A multi-method approach to understanding underachievement. Invited presentation to doctoral students and faculty, University of Louisville Department of Counseling and Human Development, Louisville, KY.

Barr, S.M. (2011). Trans* 101: A training for students, faculty, and administrators. Invited presentation to undergraduate students, faculty, and staff, Hendrix College, Hendrix, AR.

\section{TEACHING EXPERIENCE}

University of Louisville, Supervisor: Kate Snyder, Ph.D.

Graduate Level

Guest Lecturer, Human Development (ECPY 605)

Adjunct Instructor, Human Growth \& Development (ECPY 507/607)

Guest Lecturer, Human Development (ECPY 605)

Teaching Assistant, Human Growth \& Development (ECPY 507/607)

Teaching Assistant, Human Growth \& Development (ECPY 607)

Teaching Assistant, Human Growth \& Development (ECPY 507/607)

Guest Lecturer, Differential Diagnosis \& Treatment (ECPY 621)

Teaching Assistant, Evaluation \& Measurement (ECPY 540)

Teaching Assistant, Evaluation \& Measurement (ECPY 540)

Spring 2016

Fall 2015

Spring 2015

Fall 2014

Spring 2014

Fall 2013

Spring 2013

Spring 2013

Fall 2012

Undergraduate Level

Guest Lecturer, Learning Theory \& Human Growth/Development (ECPY 107) Fall 2014

\section{Additional Relevant Professional EXPERIEnCe}

February 2015-present Social Media and Website Manager

Kathy Barr Counseling \& Consulting, PLLC

Nov 2011-Jul 2012

Trans* Youth Advisor

Community Action Youth Programs

AWARDS

2017

Recipient

WPATH Student Research Award and Grant

2017

Recipient 
USPATH Inaugural Meeting Scholarship

2014

Recipient

APA Division 44 Transgender Research Award

2013

Semi-Finalist

Point Foundation Scholarship

Professional Service

2018-ongoing

Editorial Activities

2017-ongoing

2017

2013-2014

University Service

2012-2014

Community Service

2013-2016

2014

2013-2014
Co-Chair, Communications Committee APA Division 44

Reviewer

Psychotherapy

Reviewer

Transgender Health

Reviewer

Division 44 Student Research Award

Department Representative

University of Louisville Graduate Student Council

Member, Board of Directors

ACLU of Kentucky

Equity Officer

ACLU of Kentucky

Mentor

Louisville Youth Group

American Psychological Association

Division 39 - Psychoanalysis

Division 44 - Society for Psychology of Sexual Orientation \& Gender Identity

Division 56 - Division of Trauma Psychology 
International Society for Traumatic Stress Studies

World Professional Association of Transgender Health 